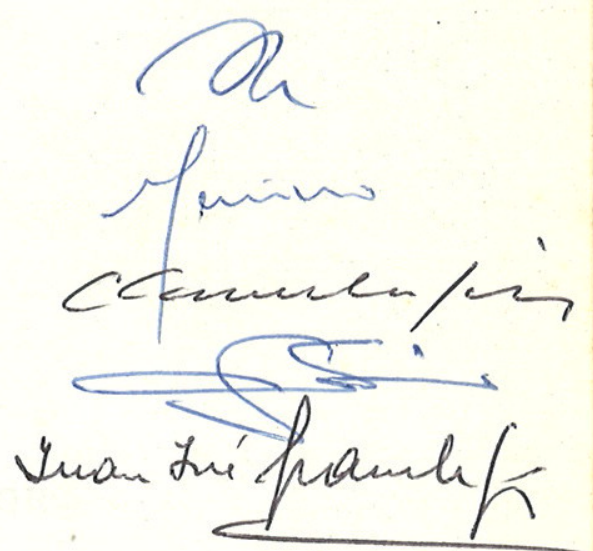

O LOOP DE WILSON EM

QUARTA ORDEM

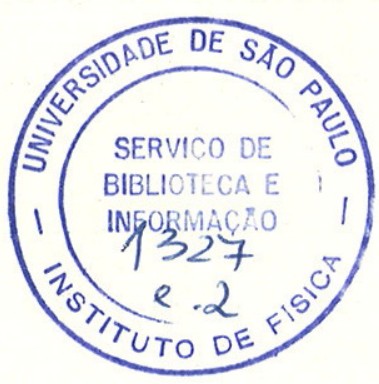

Rubens de Melo Marinho Junior

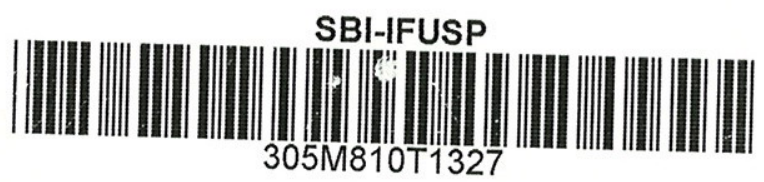

INGTITUTO DE FISICA

UNIVERSIDADE DE SAO PAULO 
RUBENS DE MELO MARINHO JUNIOR

\title{
O LOOP DE WILSON EM QUARTA ORDEM
}

\author{
Tese apresentada ao \\ Instituto de Física da \\ Universidade de São Paulo \\ para a obtenção do \\ título de Doutor
}

SÃO PAULO

DEZ - 1983 


\section{FICHA CATALOGRAF ICA}

$$
\begin{aligned}
& \text { Preparada pela Biblioteca do } \\
& \text { Instituto de Fisica da Universidade de Säo Paulo }
\end{aligned}
$$

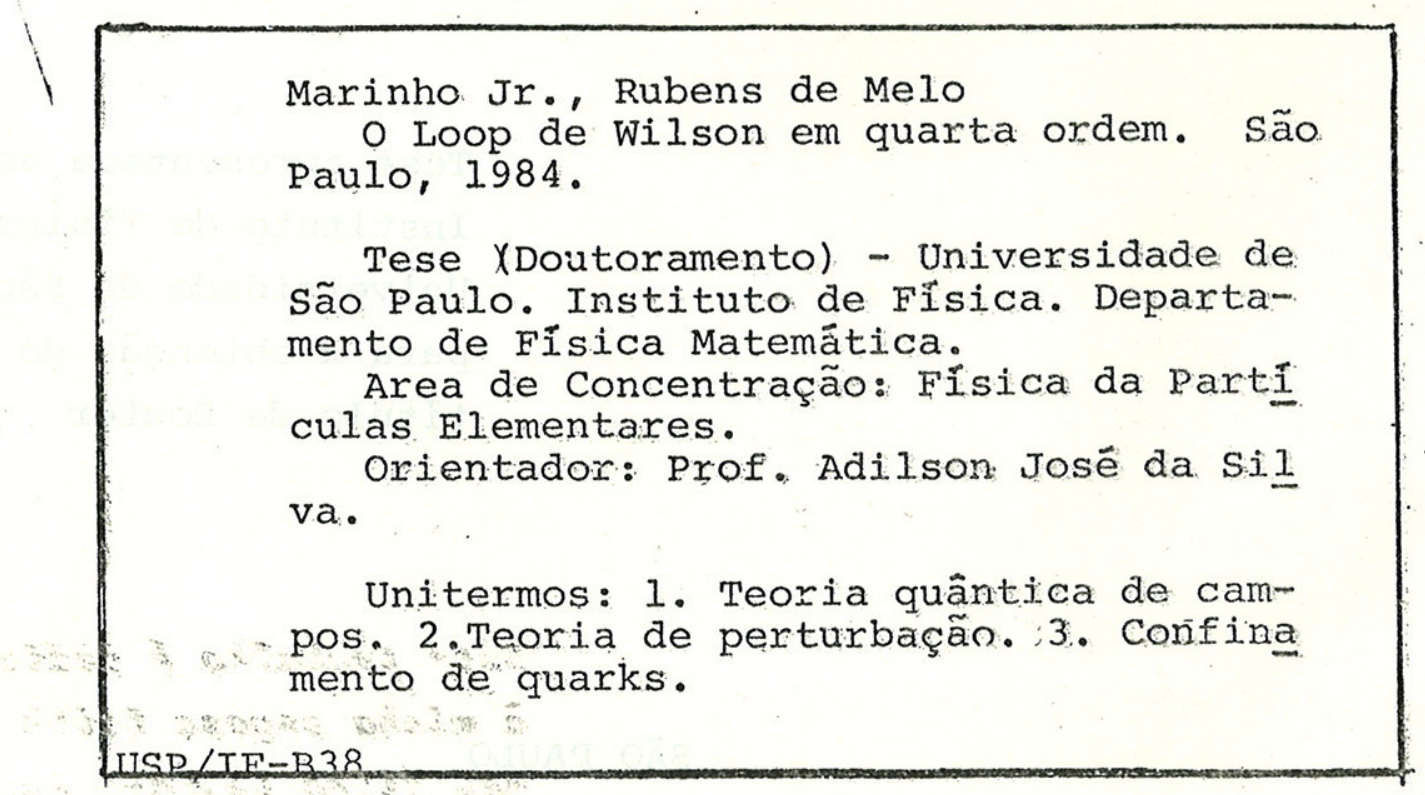




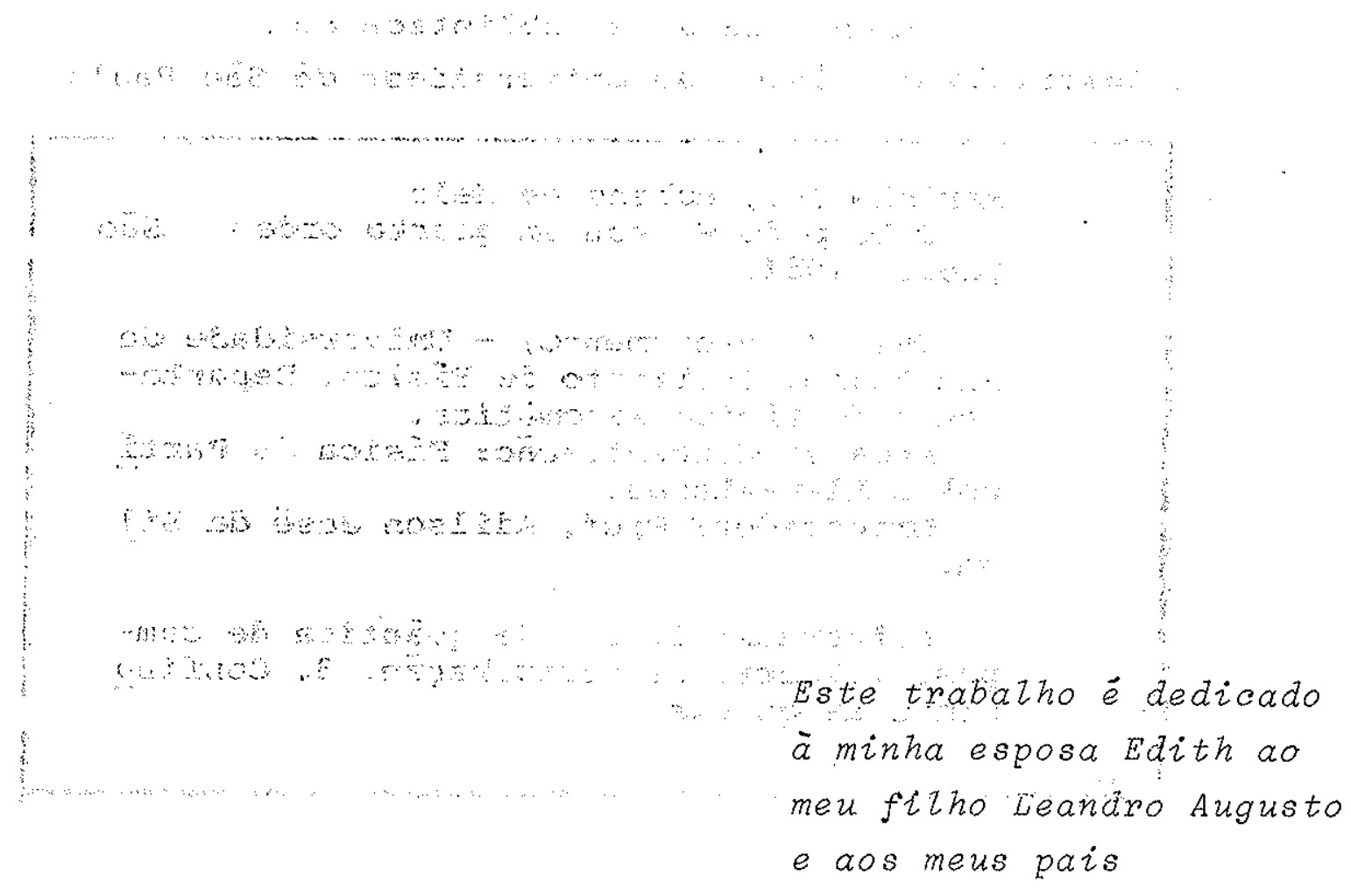


HOMENAGEM POSTUMA

Presto esta homenagem ao

Prof. Dr. Josē Alberto Albano do Amarante,

que muito contribuiu para a minha formação.

Foi meu orientadox desde a Iniciação

Cientifica atē o início dos trabalhos para

o doutoramento, ocasião do seu falecimento.

Era uma pessoa enérgica, pragmática, sincera,

cordial e com grande senso de justiça, sempre

dispensando elevada consideração aos seus

orientados. As nossas reuniões semanais

serviram de estímulo e entusiasmo ao

prosseguimento dos meus trabalhos. Fica portanto de püblico o reconhecimento pelos beneficios que recebi e a gratidão para com este com quem tive - previlégio de conviver e cuja memória em mim permanecerá. 
Aos professores Adilson José da Silva e Juan José Giambiagi pela orientação, amizade e consideração, sem pre me atendendo tardes inteiras em prejuizo de seus afaze res. As sugestões dadas, criticas construtivas, o apoio e o incentivo nas horas de desânimo muito contribuiram para o aprimoramento do trabalho. Sou grato ainda pela liberdade dada na escolha dos rumos e mëtodos para a consecução deste trabalho e pelo tratamento cordial, considerando-me como co lega de trabalho em vez do relacionamento aluno-professor.

Aos professores José Roberto Leite e Henrique Fleming pelo perído em que me orientaram formalmente.

Ao professox Abel Rosato, meu conselheiro na época em que estudava no ITA, que percebeu minhas tendên cias cientificas e que me colocou em contato com o Cel Ama rante.

Ao Rogério Lopez Garcia por ter lido parte dos manuscritos, pelo periodo que trabalhamos juntos utilizando a linguagem simbölica scHOONSCHIP e pelos conselhos, princi palmente aqueles que geravam lucro.

Ao Jair Lucinda por ter lido parte dos manus critos, pelos períodos de estudo em conjunto e discussões sauaāveis.

Ao Cesar Linhares pelo companheirismo, incenti vo, discussões produtivas e pelas caronas.

Aos demais amigos do IEAv que de uma forma ou de outra influenciaram durante esse periodo. 
A minha esposa Edith V.A. Marinho pela atenção, tolerância e apoio nas horas dificeis. Pela compreensão que teve quando eu abandonava o lax para trabalhar durante a noj. te nos fins de semana e feriados.

A Rita, secretāria da pós-graduação do IFUSP pelo atendimento eficiente e atencioso.

à Célia Regina Rosa por ter tido a paciência e a boa vontade de datilografar este trabalho, o qual foi feito com estētica e elegância.

Ao CPD do IEAv/CTA pela eficiência do trabalho de seus integrantes, muitas vezes nos deixando constrangi dos quando observávamos que eles paravam os seus trabalhos para cuidar dos nossos. Em especial agradeço ao Valentim Novackoski por ter alterado o compilador do PASCA.L, para que este ficasse compativel com a saída gerada pelo REDUCE2; ao chefe da operação Bolis e ao operador Mariotto.

Finalmente agradeço ao suporte financeiro do IEAv/CTA dado através do meu contrato de pesquisador, que aliás é a unica instituição do país que considera uma tese como trabalho profissional. 
o décimo primeiro mandamento

Herdaxás o solo sagrado e a fertilidade será transmitida de geração em geração. protegerás teus campos contra a erosão e tuas florestas contra a desolação e im pedirās que tuas fontes sequem e que teus campos sejam devastados pelo gado, para que teus descendentes tenham abun dância para sempre. Se falhares, ou al guém depois de ti, na eterna vigilância de tuas terras, teus campos abundantes se transformarão em solo estéxil e pe dregoso ou em grotões äridos, teus des cendentes serão cada vez menos numero sos, viverão miseravelmente e serão eli minados da face da terra.

Dr. Walter Clay Lowdermil 
- loop de Wilson se apresenta como uma variā vel muito apropriada para servix de base para uma teoria das forças nucleares. Esta afirmação é justificada se lem brarmos que a apresentação usual da cromodinâmica quântica perturbativa parte de variäveis coloridas, um fato altamen te criticavel, pois elas não representam qualquer variável física, enquanto que na eletrodinâmica as variāveis que des crevem os elétrons e fótons são físicas.

Esta possibilidade de estudar as interações fortes jmplica na necessidade de um claro entendimento da estrutura analitica dos loops de Wilson.

o objetivo deste trabalho é usar a tëcnica da regularização dimensional para discutir a estrutura dos dia gramas da expansão perturbativa do loop de Wilson em quarta ordem. A introđução do nümero de dimensões como variāvel an-litica, isola as divergências sob a forma de polos no plano complexo da dimensão, facilita a discussão da renorma lização, além de proporcionar um método invariante de gauge.

Com esta técnica renormalizamos a constan te de acoplamento das interações fortes até a quarta ordem e mostramos que em quatro dimensões o resiauo não depende nem da curva nem do arco no qual ele é calculado. Também mos tramos que é possivel calcular a parte finita do loop de Wilson para o caso especial de duas semi-retas de mesma ori gem. 


\section{ABSTRACT}

The wilson loop shows itself as a very appropriate variable to be used as a base in a theory of nucleax forces. This statment is justified if we remember that the usual presentation of perturbative quantum chromodynamics starts from colored variables, a fact itself subject to critism since they do not represent any physical variable, whereas in the usual quantum eletrodynamics the variables that describes eletrons and photons are physical.

This possibility of studying strong interactions implies the need of clear a understanding of the analytical structure of the Wilson loops.

The purpose of this dissertation is to use the technique of dimensional regularization to discuss the structure of perturbative expansion diagrams of the Wilson loops up to fourth order. The introduction of the number of dimensions as an analytical variable isolates the divergences in the form of poles in the complex plane of the dimensions, make the renormalization procedure easiex and also makes the method gauge invariant.

With these techniques we have renormalized the coupling constant of strong interactions up to fourth order and have also show that in four dimension the residue is dependent neither on the curve nor on the arc on which it is calculated. We have also shown that it is possible to calculate the finit paxt of the Wilson loops for the special. case of two half lines with the same origin. 


\section{INTRODUC $\widetilde{A} O$}

o loop de wilson se apresenta como uma variá vel adequada para tratar a cromodinamica, principalmente no que diz respeito ao confinamento.

Neste trabalho nos propusemos a renormalizax a constante de acoplamento das interações fortes até a quarta ordem utilizando a regularização dimensional. A fim de con cretizar tal objetivo, devemos mostrar que o resíduo obtido no cálculo do loop de wilson independe da curva e do arco, se aquela for lisa. Essa demonstxação está apresentada no capitulo 4. Os cál.culos dos diagramas são extensos e compli cados a ponto de justificar a utilização da linguagem simbó lica SCHOONSCHIP [0.I]. Essa linguagem foi escrita por Veltman com a finalidade de se calcular diagramas de Feynman.

Calculamos o fator de fase quântico para a fa mília de hipërboles que têm em comum duas semiretas como as sintotas.

Fm continuidade ao trabalho de Abud, Bollini e Giambiagi [4.3], calculamos em quarta ordem o loop de wilson para a circunferência.

A seqüência de apresentação desse trabalho é a seguinte:

- No capitulo um damos uma idéia do que seja o loop de Wilson, mostramos que ele é invariante por transforma ções de gauge, o seu papel no contexto do confinamento alëm de apresentar motivações para o seu uso.

- No segundo capitulo, fizemos um breve resumo sobre a regularjzação dimensional, a qual foi usada para a re normalização da carga. 
- No terceiro capitulo, deduzimos as regras de Feynman para o loop de Wilson.

- No quarto capitulo, renormalizamos a carga da cromodi nâmica quäntica em quarta oxdem.

- No quinto capitulo, mostramos que o resíduo que se ob tém do cálculo do loop de Wi.lson para curvas lisas, em quatro dimensões, não depende do arco nem da cur va. Mostramos, tambëm, que é possivel calcular a par te finita do fator de fase quântico para duas semire tas de mesma orj.gem.

- No sexto capitulo, calculamos o loop de Wilson paxa a circunfexência em quarta ordem.

- Finalmente, no último capítulo, apresentamos as con clusões. 


\section{INDICE}

päg.

CAPETULO I - CONSIDERACÖES PREITMINARES $\ldots \ldots \ldots \ldots \ldots$ I

1.l - Teorias de gauge $[1.1] \ldots \ldots \ldots \ldots \ldots \ldots \ldots \ldots$

1.2 - Motivações para introdução do conceito do fator de fase não integrável ................. 4

1.3 - O fator de fase não integrável em teorias de gauge não abejianas .................... 8

1.4 - O loop de Wilson é invariante portransformações

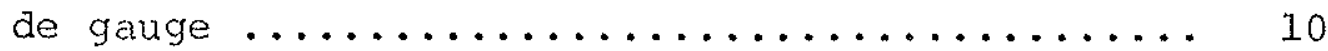

1.5 - O critério de wilson para o confinamento de

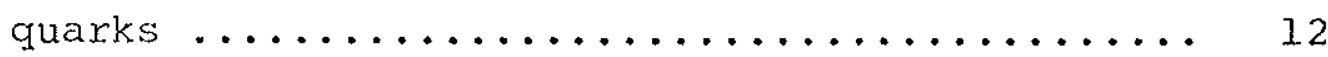

CAPITUIO 2 - A REGULARTZAÇÕO DTMENSIONAL $\ldots \ldots \ldots \ldots \ldots$

2. I - Divergências ultravioleta em teorias quânticas. 16

2.2 - Fundamentos matematicos .............. 18

2.3 - Prescrições para se regularizar dimensionalmen

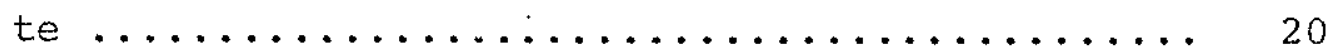

2.4 - Algumas relações üteis .............. 22

2.5 - Extensão da regularização dimensional para cam pos sem massa ........................ 23

CAPITULO $3-$ OBTENGÃO DOS DIAGRAMAS $\ldots \ldots \ldots \ldots \ldots \ldots$

3.1 - Obtenção do $100 p$ de wilson na representação de

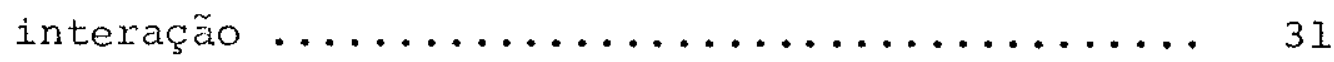

3.2 - Regras de Feynman ................... 33

3.3 - Regras de Feynman para o loop de wilson ...... 37

3.3 .1 - o propagadox de glúon ............... 39

3.3 .2 - o propagador do fantasma .............. 40

3.3 .3 - o vêrtice de três glúons ............... 40

3.3 .4 - o vertice de quatro gltions ............. 43

3.3 .5 - o vertice com fantasmas .............. 45

3.3 .6 - o vêrtice no contorno .............. 45 
Päg.

3.4 - Fatores combinatórios ................ 46

3.5 - Inxpressões dos diagramas de 4 a ordem $\ldots \ldots \ldots .48$

CAPITULO 4 - CALLCULOS DOS DTAGRAMAS .......... 57

4.3. - Anălise das divergências ................ 57

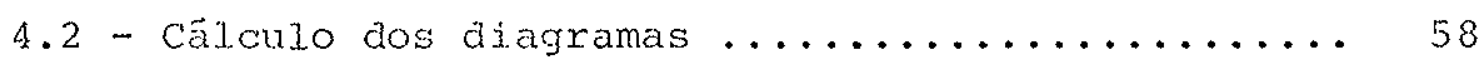

4.2 .1 - Diagrama de segunda ordem ............... 59

4.2 .2 - Girino com duas pernas em quarta ordem ..... 60

4.2.3 - Cálculo do diagrama do gixino com uma perna.. 61

4.2 .4 - Cájculo do diagrama com fantasmas ......... 62

4.2 .5 - Cálculo dos dilagramas de dois glüons não inte

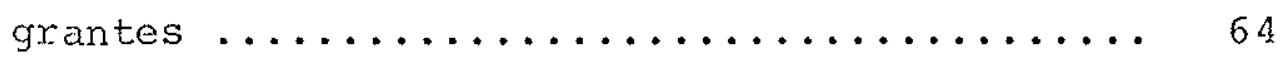

4.2.6 - Cálculo do diagrama com um vértice de três

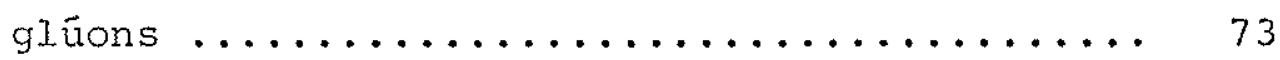

4.2 .7 - Cálculo do diagrama de dois vértices com três

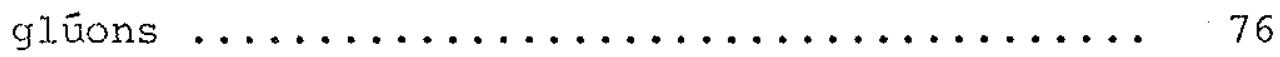

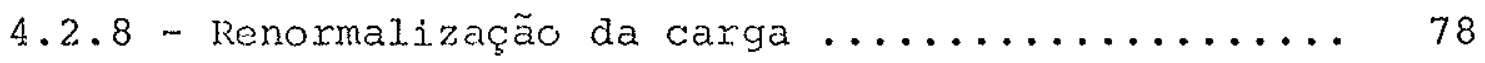

CAPITULO 5 - PROVA DA INDEPENDENCIA DO RESIDUO EM RE LACÃ̃ AO ARCO E A CURVA E CALCULO DA HIPERBOLE EM SEGUNDA ORDEM $\cdots \cdots \cdots \cdots \cdots$

5.1 - Independência do resỉduo em relação à curva e ao

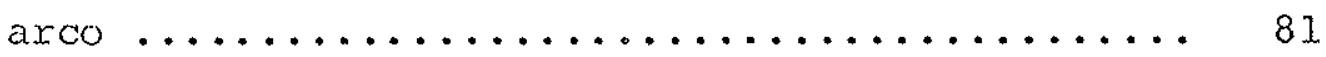

5.2 - Cálculo de $V(C)$ para a hipérbole $\ldots \ldots \ldots \ldots \ldots .6 .68$

CAPITULO 6 - CALC. PARTES FINITAS PARA A CIRCUNFERENCIA 95

6.1 - Integral necessária para o cálculo dos diagra mas $G_{4+}, G_{4 b}, G_{4 c}$ do capítulo quatro ......... 95

6.2 - Resolução da integral do diagrama $G_{49}$ sobre a cixcunferência $\ldots \ldots \ldots \ldots \ldots \ldots \ldots \ldots \ldots . . \ldots \ldots$

6.3 - Cálculo das integrais da expressão $\mathrm{k} \ldots \ldots \ldots . . .100$

CAPITULO $7-$ CONCLUSOES $\ldots \ldots \ldots \ldots \ldots \ldots \ldots \ldots \ldots \ldots$ 
Päg.

REFEREMCTAS BIBTIOGRATTCAS $\ldots \ldots \ldots \ldots \ldots \ldots \ldots \ldots, 125$

APENDTCE A - ALGUMAS IN'TEGRAIS OTEIS

APENDTCE B - CALLCULO DO DTAGRAMA DE UM VERTTCE COM TRES GLOONS

APENDTCE C - CATLCULO DO DIAGRAMA DE DOIS VERTICES COM TRES GLUONS 


\section{CAPITULO 1.}

\section{CONS TDERACÕES PRETIMMTNARES}

Este capitulo tem por finalidade fazer um histörico dos loops de wilson, dax notivação para o seu uso mostrando como esse novo conceito pode servir de base para uma formulação mais satisfatōria do que a cromodinâmica quântica.

Na seção 1.J temos alguns aspectos das teo rias de gauge. Mostramos como esse conceito evolui a par tix da eletrodinâmica e se generalizar para grupos de sime tria que não $O U(1)$.

Na seção 1.2 apresentamos a ambiguidade' da formulação diferencial das teorias de gauge, mostrando que situações físicas diferentes podem sex descritas pelo mes mo tensor de intensidade dos campos, para regiões multipla mente conexas. Impõe-se então o desenvolvimento de um novo conceito para o tratamento dessas teorias.

$\mathrm{Na}$ seção 1.3 discorremos brevemente sobre os trabalhos atuais, principalmente os de Migdal e Makeenko, a firn de mostrar a aplicação desse conceito em teorias de gauge não abelianas.

Na seção 1.4 mostramos que o loop de Wilson ë invariante sob transformaçŏes de gauge, e na ültima se ção falamos sobre critério de confinamento de Wilson. 
3. . - TEORTAS DE GAUGE* $[1,1]$

Há fortes indicios de que a natureza seja des crita pelas teorias de gange. Esta opinião é baseada nos seguintes fatos:

a) a única teoria completamente contirmada experimen talmente, a eletrodinâmica quântica, è um caso par ticular dast teorias de gauge;

b) a formulação de modelos fenomenológicos das intera ções fracas, adquixiram elegância e consistência com - formalismo das teorias de gauge. A interação fe nomenológica de quatro férmions, foi substituida pe la interação com bosons vetoriais intermediários, que são os campos de gauge de uma teoria de YangMi.1.1s;

c) tem-se mostrado que os modelos fenomenológicos com quarks se apresentam mais naturais no contexto das teorias de gauge. Alêm disso as teorias de gauge são a unica possibilidade conhecida de descrever o fenômeno da liberdade assintötica no formalismo das teorias de campos. Estas teorias tem recursos para explicar o fenômeno do confinamento dos quarks, ape sax do mecanismo correspondente disto não estar mui to claro atualmente;

d) finalmente, é possivel considerax a gravitação como uma teoria de gauge. * Weyt foi o primeiro a introduzir o termo "transforma
soes de gauge" numa tentativa de unificar o eletromagne tismo com a gravitąăo. 
Teorias de gauge são aquelas cujas Jagrangia nas são invariantes por transformargões do tipo

$$
\phi(x) \rightarrow e^{-i g \lambda_{\alpha}^{a}(x)} \phi(x)
$$

onde $\phi(x)$ è qualquer campo, $\alpha^{a}$ è uma função arbitrāria, $\lambda^{a}$ são os geradores do grupo de simetria da lagrangiana, e $g$ uma constante de acoplamento.

No caso simples do grupo de simetria ser o $\mathrm{U}(1)$ e, $g$ a carga do elétron, obtemos a eletrodinâmica im. pondo que a lagrangiana seja invariante por transformações de gauge locais. A eletrodinâmica vista deste modo foi in troduzida por weyl em 1929 [1.2].

A obtenção do tensor das intensidades de cam po a partir da invariância de gauge local das teorias esta exaustivamente estudado nas referências [1.3], [1.4], [1.5].

Aqui. somente relembramos que o acoplamento dos campos de gauge $A_{\mu}^{a}$ com os campos de matéxia $\psi \vec{e}$ obtido pela seguinte prescrição: substituindo $\partial_{\mu}$ pela derivada co variante $[1.6]$.

$$
D_{\mu} \equiv\left(\partial_{\mu}+i g A_{\mu}^{a} \lambda^{a}\right)
$$

A teoria invariante pelo grupo de simetria Su(2) foi primeiramente estudado por Yang-Mills em 1954 [1.7] e dai por diante todas as teorias invariantes por transformações de gauge e cujo grupo de simetria é não abe liana recebem os seus nomes. A lagrangiana de Yang-Mills tem a forma

$$
L_{X M}=-\frac{1}{4} F_{\mu \nu}^{a} F^{a \mu \nu}+L_{0}\left(\psi, D_{\psi}^{\mu}\right)
$$


onde

$$
F_{\mu \nu}^{a}=\partial_{\mu} A_{\nu}^{a}(x)-\partial_{\nu} A_{\mu}^{a}(x)-g t^{a b c} A_{\mu}^{b}(x) A_{\nu}^{c}(x)
$$

e

$$
D^{\mu} \psi(x) \equiv\left[\partial^{\mu}+i g A^{\mu}(x)\right] \psi(x)
$$

são as constantes de estrutura do grupo. ${ }^{\mathrm{F}_{\mu \nu}}{ }_{\mu \nu}^{\mathrm{a}}$ o tensor das intensidades de campo.

No nosso caso o grupo de simetria ē o $\mathrm{Su}(3)$, sendo assim temos que os campos $A_{\mu}^{a}(x)$ são os campos de glüons, $g$ è a constante de acoplamento das interações for tes $e \psi(x)$ são os campos dos quarks.

\section{2 - MOTIVACOEES PARA INTRODUÇÃO DO CONCEITO DO FATOR DE FASE NÃO INTEGRAVEL}

O conceito do eletromagnetismo foi proposto por Maxwell e Faraday para descrever efeitos eletromagnët cos numa região do espaço tempo. De acordo com este concei to a intensidade de campo $\mathrm{F}_{\mu \nu}$ descreve os fenômenos eletro magnéticos. Concluiumse mais tarde [1.8] que $F_{\mu \nu}$ sozinho, na teoria quântica, não conseguia descrever completamente os efeitos eletromagnêticos da função de onda do elêtron. O experimento de Aharanov - Bohm, primeiramente realizado por Chambers [1.9], mostrou que se um feixe de elëtrons for emi tido em uma região onde $F_{\mu \nu}=0$, exceto em um buraco, como mostra a figura 1.1, convém salientar que a região é multi plamente conexa, isto $\bar{e}$, o buraco onde $\mathrm{F}_{\mu \nu} \neq 0$ não pertence à região. Isto significa que o elêtron está sempre na re gião onde $F_{\mu \nu}=0$. 


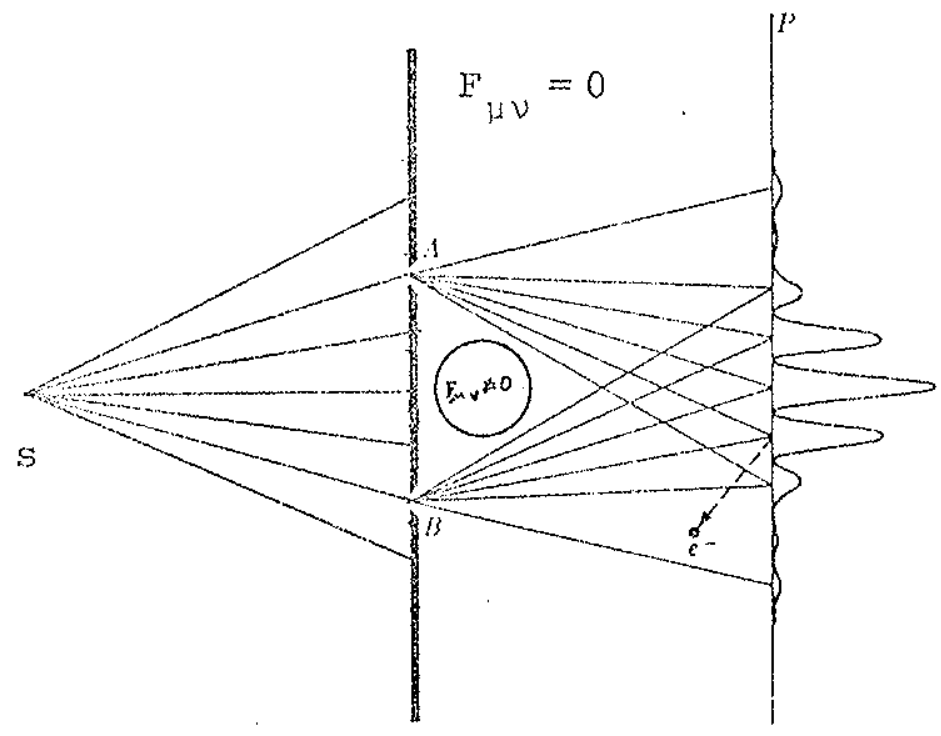

fig. 1.1

então as franjas de interferência vão depender do fator de fase (chamado pelos matemáticos de fator de fase não inte gräve L)

$$
e^{\frac{i e}{\not{X} C}} \oint A_{\mu}(x) d x^{\mu}
$$

Para mostrar esta dependência vamos precisar da função de onda $\psi$ do elëtron na região de interferência. Consideramos então uma solução da equação de schrödinger na região onde o vetor potencial $A_{\mu}$ è diferente de zero, a qual è dada por

$$
S=\psi_{i}^{0} e^{\frac{j e}{\not h C}} \int_{(i)} A_{\mu}(x) d x^{\mu}
$$

onde $\psi_{i}^{0}$ é a solução da equação quando $A_{\mu}(x)=0$ e i. é o ca minho percorrido pelo elètxon.

portanto na região de interferência da fig. 1.1 a função de onda tem a forma

$$
\psi=\psi_{1}^{0} e^{\frac{i e}{\not h C}} \int_{(1)} A_{\mu}(x) d x^{\mu}+\psi_{2}^{0} e^{\frac{i e}{\not h C}} \int_{(2)} A_{\mu}(x) d x^{\mu}
$$


e

$$
\begin{aligned}
|\psi|^{2}=\left|\psi_{1}^{0}\right|^{2}+\left|\psi_{2}^{0}\right|^{2} & +\psi_{1}^{*} \psi_{2} e^{-\frac{i e}{\not L C} \oint A_{\mu}(x) d x^{\mu}}+ \\
& +\psi_{2}^{*} \psi_{1} e^{\frac{i e}{\not / C}} \oint A_{\mu}(x) d x^{\mu}
\end{aligned}
$$

ou

$$
|\psi|^{2}=\left|\psi_{1}^{0}\right|^{2}+\left|\psi_{2}^{0}\right|^{2}+2 \operatorname{Re}\left(\psi_{2}^{*} \psi_{1} e^{\frac{\text { ie }}{\text { j/c }} \oint A_{\mu}(x) d x^{\mu}}\right) .(1.9)
$$

Se fizermos uma transformação de gauge nos campos $\psi$ e $A_{\mu}$ como segue

$$
\begin{gathered}
\psi^{\prime}=U^{-1} \psi=e^{i \alpha} \psi, \\
A_{\mu}^{\prime}=A_{\mu}-\frac{i \not C_{C}}{e} U \partial_{\mu} U^{-1}
\end{gathered}
$$

ou

$$
A_{\mu}^{\prime}=A_{\mu}-\frac{\underline{\prime \prime c}}{e} \partial_{\mu}^{\alpha},
$$

teremos na região de interferência,

$$
\left|\psi^{\prime}\right|^{2}=\left|\psi_{1}^{0}\right|^{2}+\left|\psi_{2}^{\prime}\right|^{2}+2 \operatorname{Re}\left(\psi_{2}^{*} \psi_{1} e^{\operatorname{ie} C} \oint A_{\mu}^{\prime}(x) d x^{\mu}\right) .
$$

Substituindo as equações (1.10) na equação (1.11) temos 
$|\psi|^{2}=\left|\psi_{1}^{0}\right|^{2}+\left|\psi_{2}^{0}\right|^{2}+2 \operatorname{Re}\left(\psi_{2}^{*} \psi_{1} e^{\frac{\operatorname{ie}}{\operatorname{dx}} \oint A_{\mu}(x) d x^{\mu}+i \oint_{\mu} a_{\mu} \alpha d x^{\mu}}\right)$.

Comparando a equação (1.12) com a equação (1.9) temos

$$
\operatorname{Re}\left[e^{i(\theta+\Omega+A)}\right]=\operatorname{Re}\left[e^{i(\theta+\Omega)}\right]
$$

onde fizemos as substituições

$$
\begin{aligned}
& \cdot \psi_{2}^{*} \psi_{1}=e^{j \Theta}, \\
& \frac{e}{\not L C} \oint_{\mu}(x) d x^{\mu}=\Omega \\
& \oint_{\partial_{\mu} \alpha(x) d x^{\mu}=\Delta .}
\end{aligned}
$$

Então temos

$$
\cos (\theta+A+\Omega)=\cos (\theta+\Omega)
$$

ou

$$
\theta+\Omega+\Delta=\theta+\Omega \pm 2 \pi n
$$

onde $\mathrm{n}$ è um nimero inteiro. Logo

$$
\Delta=2 \pi n
$$

Finalmente temos ao integrax a ültima das equações

(1.10) no contorno delimitado pelos caminhos (1) e (2) da fig. 1.I 


$$
\Omega^{\prime}=\Omega+2 \pi r \frac{\not K c}{e}
$$

onde o fluxo magnëtico $\Omega$ ' é definido pela equação (1.15) substituindo-se $A_{\mu}$ por $A_{\mu}^{\prime}$. Como os fluxos magnéticos $\Omega^{\prime}$ e $\Omega, s a ̃ o$ mensuräveis temos duas situações físicas diferentes descritas pelo mesmo tensor $F_{\mu \nu}$; portanto a formulação di ferencial subdescreve o eletromagnetismo.

\section{3 - O FATOR DE FASE NÃO INTEGRAVEL EM TEORTAS DE GAUGE NÃO ABELTANAS}

A cromodinâmica quântica trata de objetos não fúsicos como quarks e glüons. A dinâmica dos glüons é des crita pelo tensor das intensidades de campos $\mathrm{F}_{\mu \nu}^{\mathrm{k}}$ que è co variante de gauge. Em contraste o fator de fase $\phi(C)$ é in variante de gauge, alëm de estabelecex um critërio natural para o confinamento. o valor esperado no väcuo desse fator de fase recebe o nome de loop de Willson, cuja expressãoé

$$
W(C)=\left\langle 0\left|e^{-S} e^{\oint_{C} A_{\mu}(x) d x^{\mu}}\right| 0\right\rangle,
$$

onde no nosso caso $S \ddot{e}$ a ação efetiva de Yanrmilis. A QCD pode ser completamente formulada em termos dos campos com postos singletos de cor

$$
\phi(C)=\frac{I}{N} \operatorname{tr} \mathrm{P} e^{g \oint_{C} A_{\mu}(x) d x^{\mu}}
$$

aqui $N$ é o nümero de cores, $\mathrm{P}$ è a ordenação de Dyson ao longo da curva, $g$ é a constante de acoplamento das intera ções fortes, $A_{\mu}(x) \equiv A_{\mu}^{a} \lambda^{a}$ onde $\lambda^{a}$ são os geradores do gru po na representação fundamental. Assim todos os observă veis e suas dinâmicas são inteiramente reformuladas em ter 
mos do fator $\theta(C)$. Essa idéia aplicada a uma rede foi de fendjac por Wilson [1.10] e as formulas apropriadas para o continuo foram derivadas por Migdal e Makeenko [1.1.1]. Pa ra um nümero finito de cores $N$, os observäveis são expres sos atravês da média de m loops

$$
W_{m}\left(C_{1}, C_{2} \ldots C_{m}\right)=\left\langle\phi\left(C_{1}\right) \phi\left(C_{2}\right) \ldots \phi\left(C_{m}\right)>\right.
$$

Grandes simplificações ocorrem nestas förmulas para um nü mexo infinito de cores, onde todos os observäveis são ex pressos por $W_{1}(C)$, pojs $W_{m}$ fatora como segue

$$
{ }^{\prime} w_{m}\left(c_{1}, c_{2}, \ldots c_{m}\right)=w_{1}\left(C_{1}\right) w_{1}\left(c_{2}\right) w_{1}\left(c_{3}\right) \ldots w_{1}\left(c_{m}\right),
$$

onde

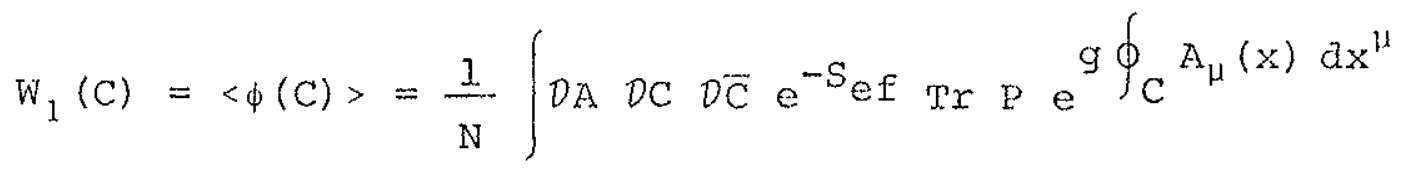

e $\mathrm{C}$ e $\overline{\mathrm{C}}$ são os campos de fantasmas.

A equação para os fatores $\phi(C)$, ajnda sem so lução para um nümero finito de cores, foi deduzido por Migdal. e Makeenko [3.12] e è a seguinte

$$
\begin{aligned}
& \frac{1}{g^{2} N} \partial_{\mu}^{x} \frac{\delta w_{m}\left(C_{1}, C_{2}, \ldots\right)}{\delta \sigma_{\mu \nu}^{l}(x)}=\oint_{C_{1}} d y_{\nu} \delta(x-y) \times \\
& \quad \times\left\{W_{n+1}\left(C_{1}{ }_{x y^{\prime}}, C_{1}{ }_{Y x}, C_{2} \ldots C_{n}\right)-\frac{1}{N^{2}} W_{n}\left(C_{1}, C_{2} \ldots C_{n}\right)\right\}+
\end{aligned}
$$




$$
\begin{aligned}
& +\frac{1}{N^{2}} \sum_{i=2}^{\infty} \int_{C_{i}} d y_{v} \delta(x-y)\left[w_{n-1}\left(c_{1}+c_{i}, c_{2}, \ldots l_{i} \ldots\right)-\right. \\
& \left.-w_{n}\left(c_{1} \ldots c_{n}\right)\right],
\end{aligned}
$$

onde $x$ é um ponto da curva $c_{1} ; c_{1}+c_{i}$ é a curva resultante da união de $C_{1}$ e $C_{i}$; $\not C_{i}$ significa que $C_{i}$ è omitido.

Esta equação para um nümexo infinito de co res $\left(\mathrm{Ng}^{2} \sim\right.$ I) Eica simplificada para

$\partial_{\mu}^{x} \frac{\delta w_{n}(c, \ldots)}{\delta \sigma_{\mu \nu}(x)}=g^{2} N \oint_{C_{1}} d y y_{\nu} \delta(x-y) w_{n+1}\left(c_{1 x y^{\prime}} C_{1 y x^{\prime}} c_{2} \ldots C_{n}\right)$.

Nesse limite

$$
w_{n}\left(C_{1}, C_{2} \ldots\right)=w_{1}\left(C_{1}\right) w_{1}\left(C_{2}\right) \ldots w_{1}\left(C_{n}\right)
$$

desde que $W_{l}(C)$ satisfaça a equação

$$
\partial_{\mu}^{x} \frac{\delta w_{1}(C)}{\delta \sigma_{\mu \nu}(x)} g^{2} N \oint_{C} d y_{\nu} \delta(x-y) w_{1}\left(C_{x y}\right) w_{1}\left(C_{y x}\right)
$$

O modo de se obter $W(C)$ nesta tese é através da teoria de pexturbação, depois de fixada o gauge.

\section{4 - O JOOP DE WILSON E INVARSANTE POR TRANSFORMACEOES DE GAUGE}

O fator de fase $\phi(C) \vec{e}$ o responsävel pelo transporte paralelo de $\psi$ de $x$ a $x '$ ao longo da curva $c$ ou seja 


$$
\psi\left(x^{\prime}\right)=\phi(C) \psi(x)
$$

onde $\psi$ ë um campo de matéria.

Se for feitia uma transformação de gauge $U(x)$ nos campos o fator de fase fica transformado do seguinte modo

$$
\psi^{\prime}\left(x^{\prime}\right)=\phi^{\prime}(C) \psi^{\prime}(x)
$$

onde $\psi^{\prime}\left(x^{\prime}\right)=U\left(x^{\prime}\right) \psi\left(x^{\prime}\right)$

e $c \dddot{e}$ a curva que liga os pontos $x \in x^{\prime}$. Temos então

$$
U\left(x^{\prime}\right) \psi\left(x^{\prime}\right)=\phi^{\prime}(C) U(x) \psi(x)
$$

ou

$$
\psi\left(x^{\prime}\right)=U^{-1}\left(x^{\prime}\right) \phi^{\prime}(C) \quad U(x) \psi(x)
$$

Uti.lizando as equações (1.29) e (1.31) temos

$$
\phi(C)=U^{-1}\left(x^{\prime}\right) \phi^{\prime}(C) \quad U(x)
$$

ou

$$
U\left(x^{\prime}\right) \phi(C) U^{-1}(x)=\phi^{\prime}(C)
$$

Se a curva for Fechada, isto é se $x^{\prime} \equiv x$ temos

$$
\phi^{\prime}(C)=U(x) \phi(C) U^{-1}(x)
$$

o loop de wilison é o valox esperado no väcuo do traço do fator de fase. Portanto

$$
\begin{aligned}
& \operatorname{Tr}\left\langle\phi^{\prime}(\mathrm{C})\right\rangle=\operatorname{Tr}\left\langle\mathrm{U}(\mathrm{x}) \phi(\mathrm{C}) \mathrm{U}^{-1}(\mathrm{x})\right\rangle \\
& \therefore \mathrm{w}^{\prime}(\mathrm{C})=\mathrm{W}(\mathrm{C}) .
\end{aligned}
$$


1.5 - O CRITERIO DE WILSON PARA O CONJINAMENTYO DE QUARKS

Se 0 loop de wilson dependex de exp (-a), onde a é a área isto implica que o potencial. entre os quarks depende linearmente da distância entre eles e, por tanto a teoria é confinante. Para justificar esse critério, iremos considerar o experimento imaginario descrito a se guix []. . 3.3].

Inicialmente separamos adiabaticamente o-par de quark $Q$ antiquark $\bar{Q}$ a uma distância relativa $R$, e a man temos assim por um tempo $T \rightarrow \infty$. Então, deixamos que os quarks se juntem e se aniquilem. A linha do universo dos quarks é mostrada na fig. 1.2

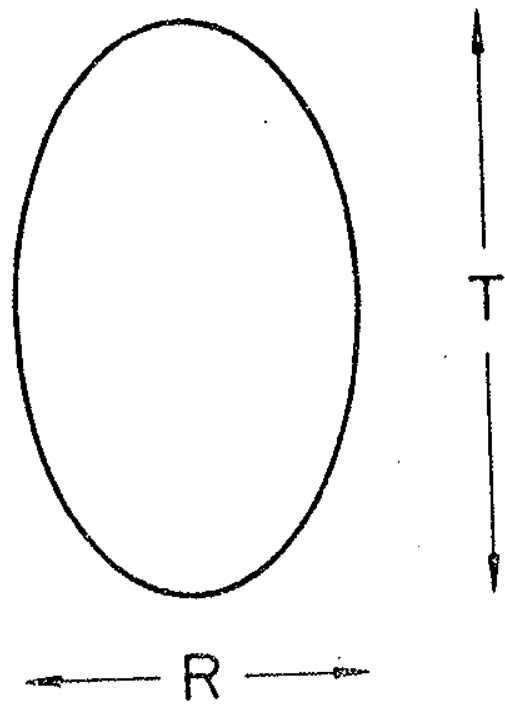

fig. 1.2

A amplitude Euclidiana para esse processo è o elemento de matriz do operador de evolução $e^{-H T}$ entre os estados ini. cial e final,

$$
<i\left|e^{-H T}\right| \mathbf{f}>
$$

onde $\mathrm{H}$ è a Hamiltoniana da cromodinâmica quântica. o opexa dor de evolução decaj no tempo e não oscila pelo fato de 
estarmos formulando o problema no espaço tuclidiano. A equa çä̊ (1.35) pode ser escrita atraves da integração funcio nal.

$$
\left\langle i\left|e^{-H T}\right| E\right\rangle=\frac{\int D A_{\mu}^{a} D C_{a} D \bar{C}_{a} e^{-S+i g} \int A_{\mu}^{a} J^{a} d^{4} x}{\int D A_{\mu}^{a} D C_{a} D \widetilde{C}_{a} e^{-S}},
$$

onde $s$ é a ação da teoria de Yang-Mijlis pura, $J_{\mu}^{a}(a=1,2, \ldots$ 8) è uma corrente externa descrevendo a linha do universo dos quarks pesados e a integraçäo funcional é sobre os cam pos de gauge e os campos de fantasmas de fadeev-Popov. Pa ra a curva da fig. 1.2 parametrizada pors, a corrente $J_{\mu}^{a}(x)$ tem a forma.

$$
J_{\mu}^{a}(x)=\oint \lambda^{a} \delta(x-x(s)) \frac{d x^{\mu}}{d s} d s
$$

Substituindo essa expressão na equação (1.36) resulta,

$$
\left\langle i\left|e^{-H T^{\prime}}\right| E>=\frac{\int D A_{\mu}^{a} D C_{a} D \vec{C}_{a} e^{-S+i g \oint A_{\mu}^{a} \lambda^{a} \frac{d x^{\mu}}{d s} d s}}{\int D A_{\mu}^{a} D C_{a} D \vec{C}_{a} e^{-S}}\right.
$$

ou

$$
<0\left|e^{-H T}\right| 0>=<e^{\dot{I g} \oint A_{\mu}^{a} \lambda^{a} d x^{\mu}>}
$$


Como os quarks estão estäticos a Hamiltoniana é simplesmente a energia potencial $V(R)$, ou seja, é a ener gia que mantém os quarks a uma distância $R . V(R)$ é obtida da expressão

$$
e^{-V(R) T}<0 \mid 0>=\left\langle e^{i g \oint A_{\mu}^{a} \lambda^{a} d x^{\mu}}>\right.
$$

Resolvendo para $V(R)$ temos

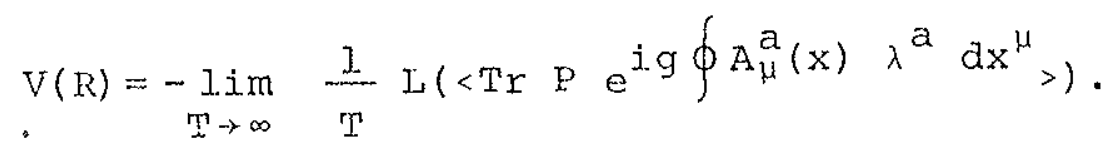

se $o$ loop de Wilson dependex da ärea, ou seja

$$
w(C)=e^{-a s}
$$

onde $s$ é a área do loop e a uma constante, temos no nosso caso

$$
V(R)=-\lim _{T \rightarrow \infty} \frac{1}{T} I\left(e^{-a R T}\right)=a R
$$

concluimos que a força de atração dos quarks è constante sendo necessário uma quantidade de energia irfinita para separä-los. No caso de $V(R)$ depender do perimetro, a força de atração daqueles é nuia, sendo possivel separä los. 


\section{CAPATULO 2}

\section{A REGULARIZACX̃O DTMENSIONAL}

A regularização djmensional foi introduzida por J.J. Giambiagi e C.G. Bollini [2.1] e t' Hooft e Veltman [2.2]. Giambiagi e Bollini observaram que a solução das equações de ondas, têm propriedades diferentes quando o nụ mero de dimensões espaço-temporais é par ou ímpar. .Como exemplo, lemoramos que o princípio de Huyghens só vale para dimensöes espaço-temporais pares. Notaram também que uma teo ria escalar tem gräficos de oxdem mais baixa finitos em es paços de dimensão impar. Como resultado obtiveram funçöes analitticas da variável $v$ (nümero de dimensões), isto os le vou a tratar as teorias quânticas usando a dimensão como um parâmetro regularizadox.

Este capitulo estā disposto da seguinte forma:

- Na Seção 2.1, mostramos o que é a reqularização dimen sional e como as civergências iniciais se refletem no parâmetro $v$.

- Na Seção 2.2 justificamos, matematicamente, a possibi lidade de se extender o nümero de dimensões do espa ço-tempo para um valor complexo.

- Na seção 2.3 damos as prescrições para se regularizar dimensionalmente uma teoria.

- Em 2.4 reescrevemos algumas relações matemāticas üteis, encontradas no artigo de Giambiagi e Bollini. [2.1] .

- E na última seção falamos sobre a extensão do método para teorias com campos sem massa. 


\section{$2 . I$ - DIVERGENCIAS ULTRAVIOLETA EM TEORTAS QUANTICAS}

Regularizações são quaisquer prescrições para tornar finitas integrais divergentes. Na regularização di mensional. o parâmetro regularizador é a pröpria dimensão do espaço-tempo. O que se faz é uma continuaçäo analítica [2.3] no nümero de dimensões do espaço-tempo $(v)$ sendo $v$ um núme xo complexo, deste modo, o resultado das integrais è uma função analïtica da dimensão, de tal forma que a divergên cia aparece como polos dessa função.

A utilidade dessa regularização, reside no fa to de que ela mantém a invariância de gauge da teoria, para qualquer valor do parâmetro v. Isso não ocorre com outras regularizações. Para ilustrar o que dissemos, considere a integral

$$
I(4) \equiv \int-\frac{d^{4} k}{(2 \pi)^{4}} \frac{1}{k^{2}\left[(k-p)^{2}+m^{2}\right]},
$$

definida sobre o espaço de momenta Euclidiano. No limite de grandes momenta $k^{2} \rightarrow \infty$, a integral $I(4)$ diverge, mas em três dimensões a integral correspondente

$$
\int \frac{d^{3} k}{(2 \pi)^{3}} \frac{1}{k^{2}\left[(k-p)^{2}+m^{2}\right]},
$$

no mesmo limite é finita. Podemos observar que integrai.s di vergentes numa dimensão, pode muito bem ser finita em outra. portanto, a idéia é: primeiro generalizar a dimensionalida de do espaço de 4 para $n$ onde $n=0,1,2 \ldots$; segundio con tinuax analiticamente de $\mathrm{n}$ para $v$, com $v$ complexo. Simboli camente 


$$
\begin{aligned}
I(4) & =\int \frac{d^{4} k}{(2 \pi)^{4}} J\left(k^{2}, k \cdot p\right) \Longrightarrow \int \frac{d^{n} k}{(2 \pi)^{n}} J\left(n, k^{2}, k \cdot p\right) \\
& \Rightarrow \int \frac{d^{\nu} k}{(2 \pi)^{\nu}} J\left(\nu, k^{2}, k \cdot p\right) .
\end{aligned}
$$

A integral I(4) não é definida, no entanto a integral $I(\nu)$ pode ser definida de tal forma que ela seja una função analitica de $v$, que em princípio pode ser calcu lada explicitamente. Uma vez que as manipulações formais en volvẹdo integrais, tais como integrações simétricas, mudan ças de variáveis e integrações por parte tenham sido feitas, nös podemos invocax o princípio da continuação analítica e voltar para o espaço quadridimensional.

Este é o momento oportuno para perguntar o significado, se hä algum, para associàr ao espaço de $v$ di mensões. Apesar de sermos incapazes, no momento, para dar mos um tal significado físico, é bom lembrar que abstrações desta natureza, não são incomuns na física. E só observar, por exemplo, que a transição da mecânica do ponto para a teoria de campos clässicos é feita substituindo $q_{j}(t)$ por $\psi_{\sigma}(x, t)$. Aqui $\sigma=0,1,2 \ldots$ denota os componentes do campo e $j=0,1,2 \ldots N$, conta $\circ$ nümero de graus de liberdade do sistema. Na transição mencionada, $q_{j}(t) \rightarrow \psi_{\sigma}(x, t)$, o indice discreto $j$ é formalmente substituido pela variāvel discreta $\sigma$ e a variāvel continua $\mathbf{x}$. Em linguagem matemätica, essa transição nada mais é que uma continuação analítica de um parâmetro espacial discreto para um contínuo. Portanto, não é de se estarrecer com o uso desse princípio matemätico. 


\section{2 - FUNDAMENTOS MATEMATICOS}

Para ajudar a esclarecer o significado da con tinuação analítica, nōs vamos, inicialmente, discutir um teorema básico e então aplicāmlo na função $\Gamma$ de Euler.

Teorema. Seja uma função analitica $g_{l}(z)$ definida numa re gião $D_{1}$ e seja $D_{2}$ outra região que tem uma e somente uma subregião $R$ em comum com $D_{1}$. Então se uma função $g_{2}(z)$ exis te e é analítica em $D_{2}$ e coincide $\operatorname{com} g_{1}(z)$ em $R$, então exis te somente uma função $g_{2}(z)$. Chamamos $g_{1}(z)$ e $g_{2}(z)$ a con tinuação analitica uma da outra. Veja figura abaixo (Knopp, 1945).

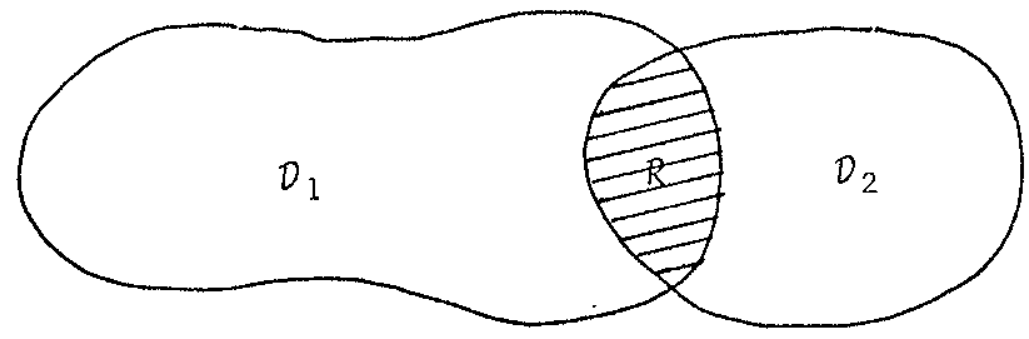

fig. 2.1

o teorema diz que $g_{2}(z)$ é única desde que $R$ não seja um conjunto vazio, $R=D_{1} \cap D_{2} \neq \phi$ ( $R$ contem uma infinidade de pontos). Ele posteriormente implica que a re presentação de $g_{1}(z)$ e $g_{2}(z)$ são iguais na subregião $R$. Fo ra de $R g_{1}$ e $g_{2}$ possuem representações diferentes.

A função $\Gamma(z), z$ complexo, aparece naturalmen te no método da regularização dimensional no cálculo de in tegrais gaussianas no espaço de momenta. A representação de $\Gamma(z)$ que nos interesse è a de Euler.

Seja a função gama de Euler $\Gamma_{E}(z)$,

$$
\Gamma_{E}(z)=\int_{0}^{\infty} d t e^{-t} t^{z-1} \quad \operatorname{Re}(z)>0
$$


que é analílica em toda área hachurada da fig. 2.2.

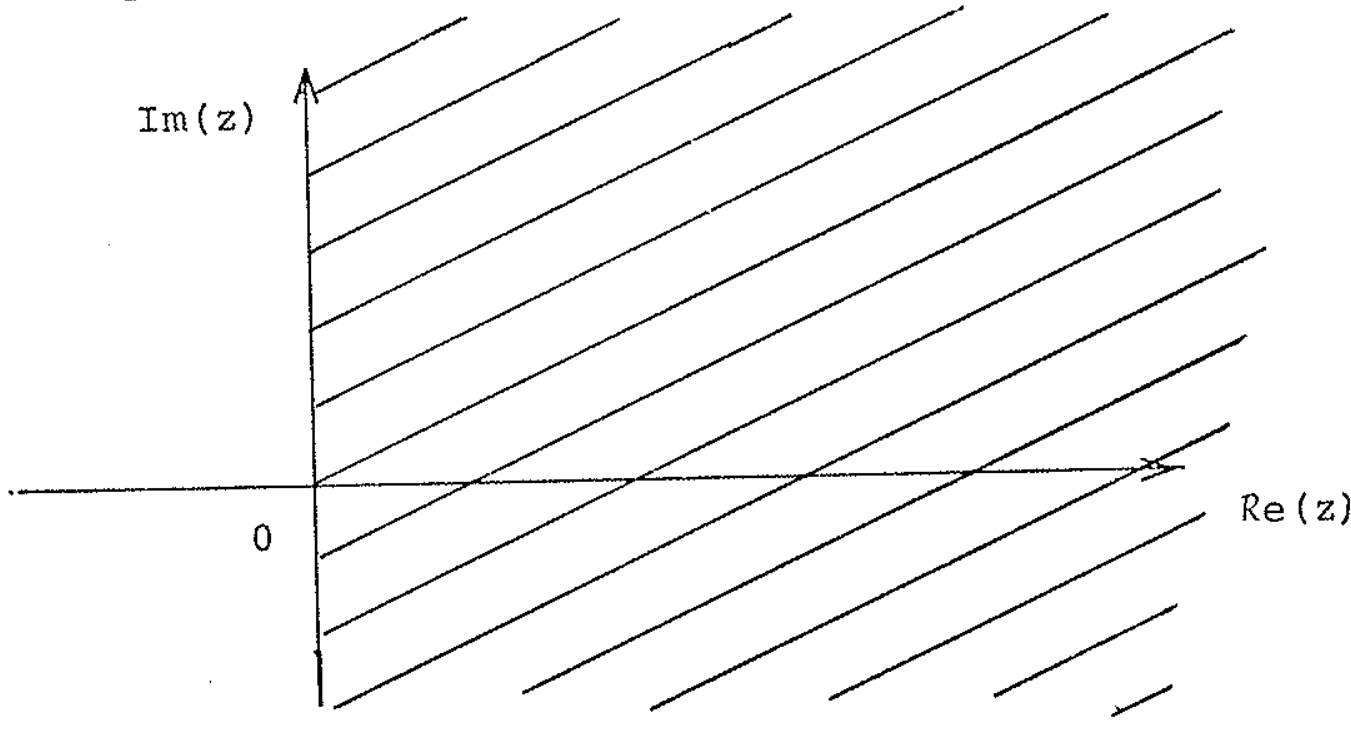

fig. 2.2

Se desejarmos um valor de $\Gamma_{E}(z)$ que está na região $\operatorname{Re}(z)<0$, será necessărio fazer uma continuação analítica dessa fun ção nessa região. Isso se consegue utilizando a fórmula de recorrência da $\Gamma$ que é

$$
\Gamma_{E}(z)=\frac{1}{z} \Gamma_{E}(z+1)
$$

Se definirmos uma $\Gamma(z)$ por

$$
\Gamma(z)=\frac{1}{\underset{\substack{n \\ m=0}}{n}(z+n)} \Gamma_{E}(z+n+1), \quad z+n \neq 0
$$

então, escolhendo adequadamente um $n$ poderemos definir $\Gamma(z)$ para quaisquer valores de $\operatorname{Re}(z)<0$. Portanto $\Gamma(z)$ é a con tinuação analítica de $\Gamma_{E}(z)$. Observe que se $\operatorname{Re}(z)>0$ então $n$ pode ser 0 , e

$$
\Gamma(z)=\Gamma_{E}(z)
$$




\section{3 - PRESCRICÕES PARA SE REGULARIZAR DIMENSIONALMENTE}

Seja a integral

$$
I(p)=\int \frac{d^{4} k}{(2 \pi)^{4}} J\left(k^{2}, k \cdot p\right)
$$

que tem divergências ultravioletas. Considere que ela está no espaço Euclidiano. Deveremos seguir os seguintes passos:

a) definimos o produto escalar para o espaço de $v$ dimen sões,

b) substituimos os propagadores, que aparecem nas ex pressões dos diagramas de Feynman em $v$ dimensões, por integrais sobre os parâmetros de Feynman ou parame trizando-os de acordo com a identidade

$$
\frac{1}{k^{2}+m^{2}}=\int_{0}^{\infty} d \alpha e^{-\alpha\left(k^{2}+m^{2}\right)}, \quad m^{2} \neq 0
$$

c) efetuamos, sobre os momenta, integrais do tipo

$$
\int \frac{a^{\nu} k}{(2 \pi)^{\nu}} e^{-x k^{2}+2 k \cdot b}=\left(\frac{\pi}{x}\right)^{v / 2} \frac{1}{(2 \pi)^{\nu}} e^{b^{2} / x}
$$

e

$$
\int \frac{a^{\nu} k}{(2 \pi)^{\nu}} \frac{\pi^{v / 2}\left(m^{2}\right)^{v / 2}-\alpha \Gamma(\alpha-v / 2)}{(2 \pi)^{\nu} \Gamma(\alpha)},
$$


e outras que poderão ser encontradas no apêndice Al. Convëm salientar que se $v$ for complexo nas integrais (2.10) e (2.11), os lados direitos passam a ser as definições delas,

d) determinamos os residuos e as partes finitas das in tegrais com as prescrições

$$
\begin{aligned}
\operatorname{Res}_{\nu \rightarrow a} f(\nu)= & \lim _{\nu \rightarrow a} \frac{1}{(n-1) !} \frac{d^{n-1}}{d \nu^{n-1}}(v-a)^{n} f(\nu), \\
& \text { considere } \frac{d^{0} f(x)}{d x^{0}}=f(x)
\end{aligned}
$$

onde $\mathrm{n}$ è a ordem do polo a

$\operatorname{pf}_{v \rightarrow a} f(v)=\lim _{v \rightarrow a} \frac{1}{n !} \frac{d^{n}}{d v^{n}}(v-a)^{n} f(v)$,

e) a amplitude resultante é uma função analítica em to do o plano complexo $v$. As divergências ultraviole tas iniciais se manifestam como polos dessa função em $v=4$. Cancelamos esses polos adicionando conve nientemente contra-termos na lagrangiana de interação original. O resultado do cälculo da amplitude fica sendo a sua parte finita,

f) finalmente, continuamos analiticamente do espaço eu clidiano para o espaço de Minkowski.

Essas prescrições são suficientes para manu seax as divergências ultravioletas; no caso de aparecer ano malias de correntes vetoriais axiais parcialmente conserva das que implicam na presença de $\gamma^{5}$, Akycampong e Delbourgo [2.5] introđuziram uma representação para esta matriz em $v$ 
dimensões. Divergências infravermelhas, que estão intimamen te Iigadas a particulas sem massa, não foram, em principio, - alvo da regularização dimensional, no entanto Leibbrandte Capper, $1974(a, b)$ [2.4], propuseram um método para tratálas, que serä discutida mais adiante.

\section{4 - ALGUMAS RELAÇÕES UTEIS}

No cälculo das integrais de Feynman nós usare mos a seguintes regras [2.1]

$$
\begin{aligned}
& \delta_{\alpha \beta} \delta_{\alpha \beta}=\nu, \\
& \delta_{\alpha \alpha}=\nu, \\
& \int d^{\nu} p p_{\alpha} p_{\beta} f\left(p^{2}\right)=\frac{\delta \beta}{\nu} \int d^{\nu} p p^{2} f\left(p^{2}\right) .
\end{aligned}
$$

para uma älgebra espinorial temos que acrescentar as regras $\left\{\gamma_{\alpha}, \gamma_{\beta}\right\}=2 \delta_{\alpha \beta}$

$$
\begin{aligned}
& \operatorname{Tr} \gamma_{\alpha} \gamma_{\beta}=d(\nu) \delta_{\alpha \beta^{\prime}} \\
& \operatorname{Tx} \gamma_{\alpha} \gamma_{\beta} \gamma_{\rho} \gamma_{\sigma}=d(\nu)\left\{\delta_{\alpha \beta} \delta_{\rho \sigma}-\delta_{\alpha \rho} \delta_{\beta \sigma}+\delta_{\alpha \sigma} \delta_{\beta \rho}\right\},
\end{aligned}
$$

onde $d(\nu)$ è uma função analítica que, para $\nu$ inteiro par, coin cide com o nümero de componentes do espinor no espaço n-di mensional, como exemplos podemos ter

$$
a(v)=2^{v / 2}
$$

ainda temos 


$$
\begin{aligned}
& \gamma_{\alpha} \not \varnothing \gamma_{\alpha}=(2-v) \not l_{1} \\
& \gamma_{\alpha} \not \varnothing \not \gamma_{\alpha}=4 p \cdot q+(2-v) \not p \not A,
\end{aligned}
$$

onde $\not \equiv \gamma \cdot p$ etc.

Outra caracteristica ütil da regularização dimensional é que nös podemos fazer uma mudança nas variä veis de integração, por exemplo, de $k_{\mu}$ para $k_{\mu}+a p_{\mu}$, logo após tenhamos feito a continuação analítica para ospaço de $v$ dimensões. Em quatro dimensões esta mudança sö é permiti da para integrais convergentes ou logaritmicamente divergen tes.

\section{5 - EXTENSÃO DA REGULARIZAÇÃO DIMENSIONAL PARA CAMPOS SEM MASSA}

o método até agora exposto é suficiente para tratax o problema das divergências ultravioletas. Esse pro cedimento no entanto, não è adequado para tratar divergên cias infravermelhas, que estão relacionadas com campos sem massa.

A tentativa de integrar

$$
\int \frac{a^{\nu} k}{(2 \pi)^{v} k^{2}}
$$

através da integral

$$
I(m, v, \alpha)=\int \frac{d^{\nu} k}{(2 \pi)^{\nu}\left(k^{2}+m^{2}\right)^{\alpha}}=\frac{\Gamma(\alpha-\nu / 2)}{(4 \pi)^{\nu / 2}\left(m^{2}\right)^{\alpha-\nu / 2} \Gamma(\alpha)},
$$


fazendo o limite quando m tende para zero, é infrutifera, pois, alëm de depender da relação entre $\nu$ e $\alpha$, que no caso de $\nu>2 \alpha$ temos uma divisão por zero, temos tambëm, em ge ral,

$$
\lim _{v \rightarrow 4}\left[\lim _{m^{2} \rightarrow 0} I(m, v, \alpha)\right] \neq \lim _{m^{2} \rightarrow 0}[\underset{v \rightarrow 4}{\operatorname{Iim}} I(m, v, \alpha)]
$$

Portanto, a integral (2.18) não pode ser deduzida, sem ambi guidade, da integral (2.19).

Um outro modo de atacar o problema é utilizar a parametrização (2.9) na integral 2.18, o que nos dá

$$
I=\int \frac{d^{\nu} k}{(2 \pi)^{\nu} k^{2}}=\int \frac{d^{\nu} k}{(2 \pi)^{v}} \int_{0}^{\infty} e^{-x k^{2}} d x,
$$

utilizando $(2.10)$ segue

$$
I=\int_{0}^{\infty} d x \frac{\pi^{\nu / 2}}{(2 \pi)^{\nu}} x^{-\nu / 2}
$$

Essa integral diverge para qualquer valor de v. Em virtude dessa divergência, não é permitido a troca das integrações feita acima.

Uma outra tentativa é escrever $(2.21)$ da se guinte forma:

$$
I=\int \frac{a^{v} k}{(2 \pi)^{v}} \frac{1}{k^{2}} \frac{(k-p)^{2}}{(k-p)^{2}}, \quad p^{2} \neq 0
$$


Desenvolvendo o numerador da integral (2.33) e resolvendo as integrais com o auxílio daquelas que estão no apênaice A. , temos

$$
\begin{aligned}
I & =(4 \pi)^{-\nu / 2}\left\{( p ^ { 2 } ) ^ { \nu / 2 - I } \Gamma ( \frac { 2 - \nu } { 2 } ) \left[\frac{2-\nu}{2} B\left(\frac{\nu-2}{2}, \frac{v-2}{2}\right)-\right.\right. \\
& \left.\left.-2\left(\frac{2-v}{2}\right) B\left(\frac{v-2}{2}, \frac{\nu}{2}\right)+\left(\frac{2-v}{2}\right) B\left(\frac{v-2}{2}, \frac{v+2}{2}\right)\right]\right\}+ \\
& +\frac{\nu}{2}(4 \pi)^{-\nu / 2}\left(p^{2}\right)^{\frac{\nu-2}{2}} \Gamma\left(\frac{2-v}{2}\right) B\left(\frac{v}{2}, \frac{v}{2}\right),
\end{aligned}
$$

onde $B$ é a função Beta.

$$
B(x, y)=\frac{\Gamma(x) \Gamma(y)}{\Gamma(x+y)}
$$

As funções gama devem ser entendidas como sendo a continui. ção analitica da gama de Tuler. Somando os termos acima utili zando a fórmula de recuxsividade das gamas, ou seja, $\Gamma(z)=$ $=(z-1) \Gamma(z-1)$, obtemos $I \equiv 0$ para qualquer $v$ ior de $v$. Es sas duas ültimas tentativas nos mostra que hā uma ambiguida de no cálculo da integral sem massa.

Para resolver esse problema Leibbrandt e Capper $1974(a, b)$ [2.4] propuseram a redefinição da integral Gaus siana generalizada:

$$
\int \frac{d^{\nu} k}{(2 \pi)^{v}} e^{-x k^{2}+2 b \cdot k} \equiv(4 \pi x)^{-v / 2} e^{b^{2} / x-x f(v)}
$$

para $x>0$, 
onde $b_{\mu}$ é um vetor do espaço de $v$ dimensões, $x$ é um nümero c. A função $f(\nu)$, que não ê única, é chamada de função de continuidade e satj.sfaz as seguintes quatro condições:

a) $f(\nu)$ é uma função analítica não nula exceto para um conjunto enumerável. de pontos;

b) $f(\nu)=0 \quad$ para $\quad \nu= \pm 0,1,2 \ldots$;

c) $f^{\ell}(v)=0$ para $\nu= \pm 0,1,2 \ldots$ e $\ell \leqslant \ell_{0}$. onde $\ell_{0}$ é finito; $\ell$ é o nümero de derivações com relação a $\vee$;

d) $\operatorname{Re}[\mathbb{F}(\nu)]>0$ para qualquer $\operatorname{Re}(\nu) \neq \pm 0,1,2 \ldots$ e pa ra alguns valores $\operatorname{Im}(\nu)$.

A ültima propriedade nos garante que a integral

$\int_{0}^{\infty} d x x^{-v / 2} e^{-x f(\nu)}$

existe.

Uma função que satisfaz as propriedades acima, pro posta por Leibbrandt e Capper [2.4] tem a forma

$f(\nu)=1-\cos (\pi \cos \pi(\cos \ldots \ldots \pi \nu)) \ldots)$

que contem $n$ cosenos encadeados, com $n$ finito. A fun ção (2.28) ainda tem as propriedades adicionais:

e) $f^{l}(\nu)=0$ para $v= \pm 0,1,2, \ldots$, onde $2^{n}>\ell+1, n$ tem o significado mencionado acima e $\ell$ tem o signifi cado que está definido em c, 
f) $f^{\ell}(v) \neq 0$ para $v= \pm 0,1,2, \ldots$ tal que $2^{n} \leqslant \ell+1$, Com essas propriedades podemos mostrar que

$$
I=\int \frac{d^{\nu} k}{(2 \pi)^{\nu}} \frac{l}{k^{2}}=0, \text { quando } \nu \rightarrow 4,
$$

então temos

$$
\begin{aligned}
I & =\int \frac{d^{\nu} k}{(2 \pi)^{\nu}} \frac{I}{k^{2}}=\int \frac{d^{\nu} k}{(2 \pi)^{\nu}} \int_{0}^{\infty} d x e^{-x k^{2}}= \\
& =(4 \pi)^{-\nu / 2} \int_{0}^{\infty} d x x^{-\nu / 2} e^{-x f(\nu)},
\end{aligned}
$$

mudando a variävel, fazendo $y=x f(v)$, temos a definição da função gama, ficando

$$
I=(4 \pi)^{-v / 2} \Gamma\left(\frac{2-v}{2}\right)[f(v)]^{\frac{v-2}{2}}
$$

Escolhendo a $f(\nu)$ com $n$ cosenos encadeados, temos pela pro priedade $f$ que $f^{\ell}(\nu) \neq 0$ para $2 \geqslant 2^{n}-1$. Sendo assim o desen volvimento de $f$ em série de Taylor em torno de $v=4$ fica

$$
\begin{aligned}
f(\nu) & =f(4)+f^{\prime}(4)(v-4)+\frac{f^{2}(4)}{2 !}(v-4)^{2}+\ldots \times \\
& \times \frac{f^{\ell}(4)}{\ell !}(v-4)^{\ell}+\frac{f^{\ell+1}(4)}{(\ell+1) !}(v-4)^{\ell+1}+\ldots= \\
& =\frac{f^{l+1}(4)}{(l+1) !}(v-4)^{\ell+1}+\ldots .
\end{aligned}
$$


Colocando $(\nu-4)^{\ell+1}$ em evidência temos

$$
\begin{aligned}
& I=(4 \pi)^{-\nu / 2} \Gamma\left(\frac{6-\nu}{2}\right) \frac{2}{(2-\nu)} \frac{2}{(4-\nu)}(\nu-4)(\ell+1)\left(\frac{\nu-2}{2}\right) \times \\
& \times\left[\frac{f^{\ell+1}(4)}{(\ell+1) !}+\frac{e^{\ell+2}(4)(v-4)}{(\ell+2) !}+\cdots\right]^{\frac{\nu-2}{2}}= \\
& =-(4 \pi)^{-v / 2} \frac{2}{(2-\nu)} \Gamma\left(\frac{6-v}{2}\right)(\nu-4)^{(\ell+1)(\nu-2 / 2)-1} \times \\
& \times\left[\frac{f^{\ell+1}(4)}{(\ell+1) !}+\frac{f^{\ell+2}(4)(v-4)}{(\ell+2) !}+\ldots\right]^{\frac{v-2}{2}} \text {, }
\end{aligned}
$$

que nos dá para $\nu \rightarrow 4, I \rightarrow 0$.

Portanto, o limite

$$
\lim _{\nu \rightarrow 4} \int \frac{d^{\nu} k}{(2 \pi)^{\nu}} \frac{1}{k^{2}}=0
$$

o que se quer dizer com esse resultado é que, no contexto da regularização dimensional, não hä inconsis tência em colocar como sendo zero essa integral altamente divergente.

o próximo passo será provar que

$$
\int \frac{a^{\nu} k}{(2 \pi)^{\nu}}\left(k^{2}\right)^{\beta-1}=0 .
$$


Paxtimos da integral (2.26), com $b=0$, e derivamos $B$ vezes em relação a $x$ a integral

$$
\int d^{\nu} k e^{-x k^{2}}=\pi^{\nu / 2} x^{-v / 2} e^{-x f(v)} .
$$

Efetuando essas derivadas, temos

$$
\begin{aligned}
\int a^{\nu} k\left(k^{2}\right)^{\beta} e^{-x k^{2}} & =\pi^{\nu / 2} x^{-\nu / 2} \sum_{j=0}^{\beta} x \\
& \times \frac{\Gamma(\beta+I) \Gamma(\nu / 2+j) E(\nu)^{\beta-j}}{\Gamma(j) \Gamma(\beta-j+1) \Gamma(\nu / 2)} x^{-\nu / 2-j} \times \\
& \times e^{-x f(\nu)} .
\end{aligned}
$$

então a integral (2.35) fica

$$
\begin{gathered}
I=(4 \pi)^{-\nu / 2} f(\nu)^{\nu / 2+\beta-1} \Gamma\left(1-\frac{\nu}{2}\right) \sum_{j=0}^{\beta} \frac{(-1)^{j} \Gamma(\beta+1)}{\Gamma(j+1) \Gamma(\beta-j+1)}=0, \\
\quad \operatorname{para} \beta=0,1,2 \ldots
\end{gathered}
$$

Na verdade quando $v / 2>\beta-1$ podemos, sem problemas, usar a integral com massa (2.19) e verificar que ela é zero no li mite $m \rightarrow 0$. Consequentemente, hã uma definição em que (2.38) vale para quaisquer $v e \beta$.

Esse capitulo foi necessärio para introduzir o método que será extensivamente utilizado no Ca pítulo 4. 


\section{OBTENCÃO DOS DIAGRAMAS}

o objetivo deste capitulo é obter as regras de Feymman para o loop de Wilson na teoria de Yang-Mills pura cujo grupo de invariância é o su(3).

Na seção 3.J partimos do loop de wilson na representação de Heisenberg para reescrevê-lo na representa ção de Dirac.

Na seção 3.2 reapresentamos as regras gerais, dadas por'thooft e veltman [3.1], para se obter os diagra mas correspondentes a uma lagrangiana.

Na seção 3.3 mostramos como se calculam os fatores de simetria para esta teoria.

E, na ültima seção, utilizando as regras apre sentadas na seção 3.2 obtivemos as expressões dos diagramas atê a quarta ordem.

Neste trabalho a rotação de wi.ck para o espa ço Euclidiano de $v$ dimensões jā está feita.

\section{1 - OBTENCẼO DO LOOP DE WILSON NA REPRESENTAÇÃO DE INTE RAÇ̃̃O}

$\mathrm{Na}$ representação de Heisenberg $\circ$ loop de Wilson tem a forma

$$
W(C)=\left\langle 0_{\text {fin }}\right| \text { Tt } \operatorname{tr} e^{\operatorname{ig} \oint_{A}^{H}(x) d x^{\mu}}\left|0_{\text {ini }}\right\rangle
$$

onde $T$ é o operador de ordenação temporal; $\mathrm{P}$ é o operador 
de Dyson que ordena pontos na curva fechada $C ; A_{\mu}^{H}(x)=$ $=A_{\mu}^{a l l}(x) \lambda^{a}$ são os campos de gauge (glüons), o sobrescrito H se refexe à representação de Heisenberg; o traço é calcu lado sobre os geradores do grupo (no caso o su(3)) $\lambda^{a}$ que es tão na representação fundamental; $g$ e a constante de acopla mento das interações fortes.

- tratamento perturbativo na representação de Heisenberg é trabalhoso, vide Roman [3.2]; portanto se faz necessário usar a representação de interação lou de Dirac), que tem como caracteristica a covariância explícita.

As relações entre os väcuos e os campos des tas duas representações são:

$$
\begin{aligned}
& \left|0_{\text {ini }}>=U(0,-\infty)\right| 0>, \\
& \left|0_{f i n}>=U(0, \infty)\right| 0>, \\
& \phi(x, t)=U(t, 0)^{-1} \phi^{H}(x, t) U(t, 0),
\end{aligned}
$$

aqui U é o operador de evolução temporal; $\left|0_{\text {ini }}\right\rangle \in\left|0_{\text {fin }}\right\rangle$ são, respectivamente, os văcuos iniciais e finais da repre sentação de Heisenberg e $|0\rangle$ é o vácuo na de Dirac e $\phi \bar{e}$ qualquer campo; vide Lurié [3.3], substituindo as relações $(3.2)$ em (3.1) teremos

$$
\begin{aligned}
W(C) & =T \operatorname{tr} P<0 \mid U(\infty, 0) U(0, t) e^{i g \oint A_{\mu}(x) d x^{\mu}} \\
& \times U(t, 0) U(0,-\infty) \mid 0> \\
& =T \operatorname{tr} P<0\left|U(\infty, t) e^{i g} \oint A_{\mu}(x) d x^{\mu} U(t,-\infty)\right| 0>.
\end{aligned}
$$


podemos mudar a ordem dos campos sobre os quais age o opera dor de ordenação temporal, para obter:

$$
W(C)=T \operatorname{tr} P<0\left|S e^{i g \oint A_{\mu}(x) d x^{\mu}}\right| 0>
$$

Na dedução da expressão anterior foram usadas as proprieda des de grupo do operador U:

$$
\begin{aligned}
& u\left(t_{1}, t_{2}\right) u\left(t_{2}, t_{3}\right)=u\left(t_{1}, t_{3}\right), \\
& U\left(t_{1}, t_{2}\right)=u\left(t_{2}, t_{1}\right)^{-1}, \\
& U\left(t_{1}, t_{2}\right)^{+}=u\left(t_{1}, t_{2}\right)^{-1}=u\left(t_{2}, t_{1}\right),
\end{aligned}
$$

onde

$$
S=U(\infty,-\infty)=T\left\{e^{\int d x L(x)}\right\}
$$

$\vec{e}$ a matriz de espalhamento e $L^{\prime}(x)$ è a lagrangiana de intera ção da teoria.

\section{2 - REGRAS DE FEYNMAN}

As definições das regras como estão apresenta das aqui são essencialmente o capitulo 2 do Diagrammar $[3 \cdot 1]$.

A lagrangiana mais geral aqui discutida $\vec{e}$ da forma

$$
\begin{aligned}
L(x) & =\psi_{i}^{*}(x) V_{i j} \psi_{j}(x)+\frac{1}{2} \phi_{i}(x) w_{i j} \phi_{j}(x)+ \\
& +L_{I}\left(\psi^{*}, \psi, \phi\right),
\end{aligned}
$$


onde $\psi_{i}$ e $\phi_{i}$ são, respectivamente, campos complexos e reais que podem sex; escalares, vetores, tensores, .... Os indi ces i simbolizam os indices de Lorentz ou de isospin ou espinor etc.. V e $W$ são operadores que podem conter deriva das e cujas transformadas de Fourier possuem inverso. O ter mo

$$
L_{I}\left(\psi^{*}, \psi, \phi\right)
$$

E um polinômio nos campos e na constante de acoplamento 9 . Podem ser não locais, isto ē, depender não só de $x$, mas de outros pontos do espaço tempo, $x^{\prime}, x^{\prime \prime}$, etc. Um termo típico de $L_{\dot{I}}$ tem a forma

$$
\begin{array}{r}
\int d^{\nu} x_{1} d^{\nu} x_{2} \ldots \alpha_{i_{1}} i_{2} \ldots\left(x, x_{1}, x_{2}, \ldots\right) \\
\psi_{i_{1}}^{*}\left(x_{1}\right) \ldots \psi_{i_{m}}\left(x_{m}\right) \ldots \phi_{i_{n}}\left(x_{n}\right) \ldots,
\end{array}
$$

onde o coeficiente a pode conter um nümero qualquer de ope radores diferenciais atuando sobre os campos.

$$
\text { A ação é definida por }
$$

$$
s=\int d^{\nu} \times L(x)
$$

Fazendo na lagrangiana as substituições

$$
\begin{aligned}
& \psi_{i}(x)=\int d^{\nu_{k}} a_{i}(k) e^{i k x}, \\
& \psi_{i}^{*}(x)=\int d v_{k} b_{i}(k) e^{i k x}, \\
& \phi_{i}(x)=\int d v_{k} c_{j}(k) e^{i k x}
\end{aligned}
$$


e

$$
\begin{aligned}
\alpha_{i_{1} i_{2}} \ldots\left(x, x_{1}, \ldots\right)= & \int d^{\nu} d^{\nu} k_{1} d^{\nu} k_{2} \ldots \\
& e^{i k x+i k_{1}\left(x-x_{1}\right)+i k_{2}\left(x-x_{2}\right) \ldots x} \\
& \times \bar{\alpha}_{i_{1}} \ldots\left(k, k_{1}, k_{2}, \ldots\right)
\end{aligned}
$$

obtemos

$$
\begin{aligned}
S & =(2 \pi)^{\nu} \int a^{\nu} b_{i}(k) \bar{v}_{i j}(k) a_{j}(k)+ \\
& +\frac{(2 \pi)^{\nu}}{2} \int a^{\nu} k c_{i}(k) \bar{w}_{i j}(k) c_{j}(k)+\ldots+ \\
& +(2 \pi)^{\nu} \int a^{\nu} k a^{\nu} k_{1} \ldots \delta\left(k+k_{1}+\ldots\right) \times \\
& \times \bar{\alpha}_{i_{1} i_{2}} \ldots\left(k, k_{i}, k_{2} \ldots\right) b_{i_{1}}\left(k_{1}\right) \ldots a_{i_{m}}\left(k_{m}\right) \ldots \times \\
& \times \ldots c_{i_{m}}\left(k_{m}\right) \ldots
\end{aligned}
$$

Das partes quadrāticas da ação tiramos os propagadores e do restante os vértices. Os propagadores, portanto, são de finidos como sendo

$$
\underset{\substack{k \\ k}}{\stackrel{j}{j}}=\Delta_{F_{i j}}(k)=-\frac{1}{(2 \pi)^{v}}\left[\bar{v}^{-1}(k)\right]_{i j},
$$




$$
\begin{aligned}
& \stackrel{j}{i \quad}=\Delta_{F_{i j}}=-\frac{1}{(2 \pi)}\left[\frac{1}{2} \bar{W}(k)+\frac{1}{2} \tilde{\bar{W}}(-k)\right]_{i j}^{-1} \\
& \text { onde } \tilde{\widetilde{W}}_{i j}=\vec{W}_{j i},
\end{aligned}
$$

enquanto os vêrtices são dados por

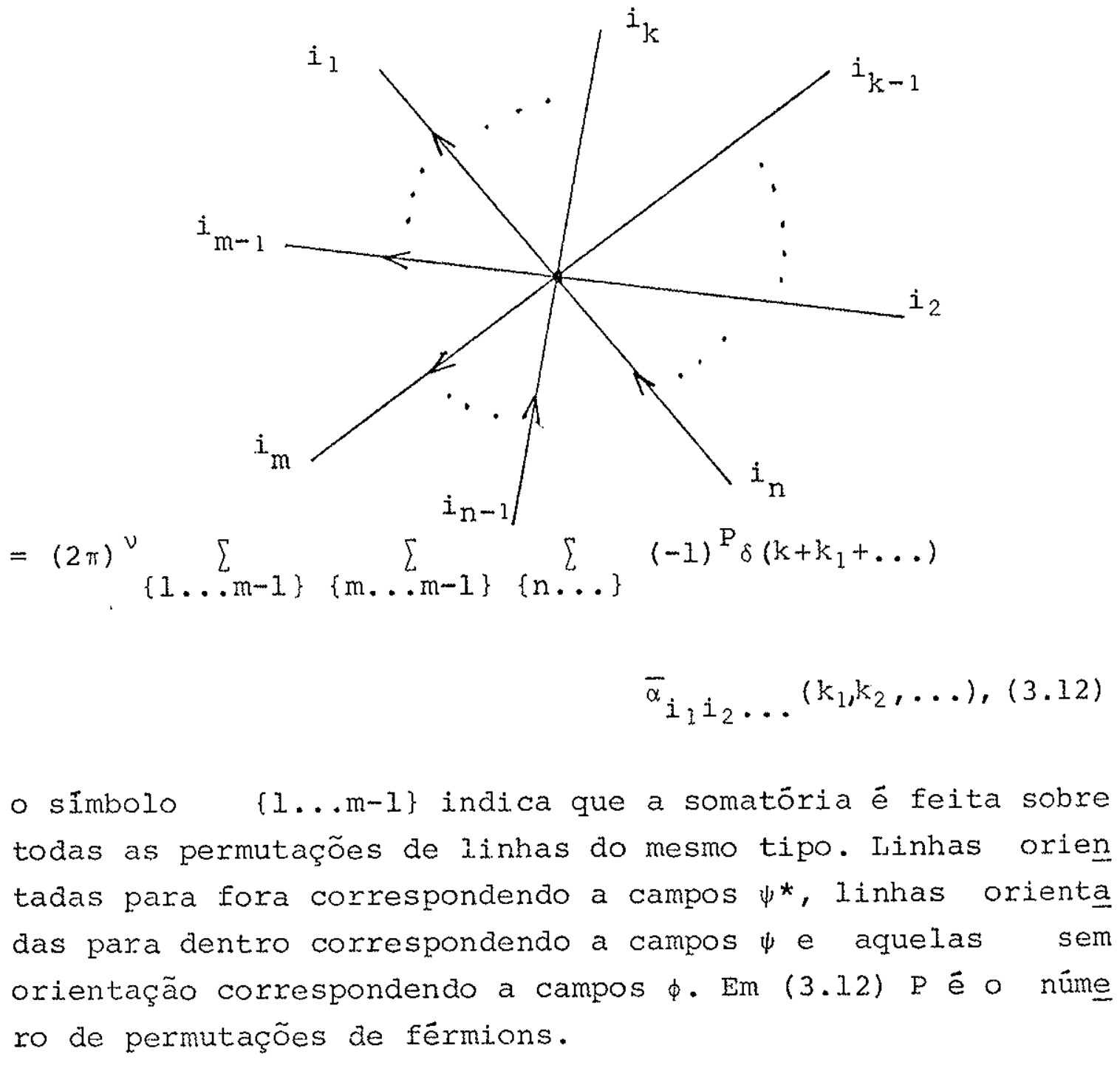

Como foi indicado, os coeficientes $\alpha$ podem ser funções de $x$, correspondendo a alguma dependência arbi trária no momentum $k$, como em (3.12). Este momentum não es tà associado com nenhuma das linhas do vértice. Se tivermos uma tal dependência em $k$, estaremos na presença de uma fon te. 
Um diagrama é obtido ligando vértices e fon tes por meio de propagadores de acordo com a orientação das linhas. Qualquer diagrama ë provido de um fator combinatô rio que corrige a dupla contagem no caso em que ocorrem par tículas idênticas. Um exemplo do cálculo do fator de sime tria pode ser visto na seção 3.4. Devem ser feitas integra ções em todos os momenta das quais os propagadores são fun ções. Se houver férmions os diagramas devem ter um sinal de acordo com as seguintes regras:

- há um sinal menos para cada loop fechado de férmions.

- diagramas que diferem pela adição ou eliminação de lí nhas de bósons tem o mesmo sinal.

- diagramas que diferem pela troca de linhas de férmions tem sinais opostos.

\section{3 - REGRAS DE FEYNMAN PARA O LOOP DE WILSON}

$\mathrm{Na}$ linguagem das integrais funcionais 0 loop de wilson è definico como

$$
W(C)=\frac{I}{N} \int D A D \bar{C} D C e^{\int d^{\nu} x L_{e E}(x)} \operatorname{TrP} e^{i g} \oint_{\mu}(x) d x^{\mu}
$$

A interação funcional é feita nos campos de gauge $A_{\mu}^{a}(x)$, cu ja dinâmica é aqui descrita pela teoria de Yang Mills pura. A ação efetiva no gauge a tem a forma [3.4]

$$
\int a^{\nu} x L_{e f}(x)=-\frac{I}{4} \int a^{\nu} x\left(\partial_{\mu} A_{\nu}^{a}-\partial_{\nu} A_{\mu}^{a}-g t^{a b c} A_{\mu}^{b} A_{\nu}^{C}\right)^{2}-
$$




$$
\begin{aligned}
& -\frac{1}{2 \alpha} \int d^{\nu} x_{\mu}\left(\partial_{\mu} A_{\mu}\right)^{2}- \\
& -i \int a^{\nu} x \partial_{\mu} \bar{C}^{a}\left(\partial_{\mu} C^{a}-g t^{a b c} A_{\mu}^{b} C^{C}\right),
\end{aligned}
$$

onde $\overline{\mathrm{C}}^{\mathrm{a}}$ e $\mathrm{c}^{\mathrm{a}}$ são os campos de fantasmas, $t^{\mathrm{abc}}$ são as cons tantes de estrutura do grupo SU(3); $N$ uma constante de nox malização; e $\lambda^{a}$, como já foi mencionado, são os geradores do grupo na representação adjunta.

As regras de comutação para os $\lambda^{a}$ são:

$$
\left[\lambda^{a}, \lambda^{b}\right]=2 i t^{a b c} \lambda^{c}
$$

e as condições de normalização para esta representação são

$$
\operatorname{Tr}\left(\lambda^{a} \lambda^{b}\right)=2 \delta^{a b}
$$

A relação

$$
t^{a b c} \operatorname{Tr} \lambda^{a} \lambda^{b} \lambda^{c}=2 i n C_{2}
$$

será usada no capitulo seguinte e é obtida facilmente das relações acima e da relação $t^{a b c} t^{a b d}=c_{2} \delta^{c d}$. Aqui $n$ é a dimensão do grupo e $\mathrm{C}_{2}$ é a constante de Casimir quadrātica.

Passamos agora, com a ajuda das prescrições dadas na seção 3.2, a obter as regras de Feynman para a teo ria de Yang-Mills, e o vêrtice do fator de fase não integrä vel. 


\section{$3.3 .1-O$ PROPAGADOR DE GLUON}

Este é obtido dos termos quadräticos nos cam pos de gange da $L_{e_{f}}$. Efetuando algumas passagens obtemos pa ra a ação correspondente, a estes termos, a seguinte expres são

$$
\begin{gathered}
I=\int d^{\nu} x\left\{-\frac{1}{2} \partial_{\mu} A_{\nu}^{a} \partial_{\mu} A_{\nu}^{a}+\frac{1}{2} \partial_{\mu} A_{\nu}^{a} \partial_{\nu} A_{\mu}^{a}-\right. \\
\left.-\frac{1}{2 \alpha} \partial_{\mu} A_{\mu}^{a} \partial_{\nu} A_{\nu}^{a}\right\} .
\end{gathered}
$$

Integrando por partes e desprezando os termos de superfi cie, pois, pela hipótese adiabática os campos vão a zero no infinito, obtemos

$$
I=\int d^{\nu} x \frac{1}{2} A_{\mu}^{a}\left\{\left(\delta_{\mu \nu} \partial^{2}-\left(1-\frac{1}{\alpha}\right) \partial_{\mu} \partial_{\nu}\right) \delta^{a b}\right\} A_{\nu}^{b}
$$

- integrando da expressĩo acima tem a forma do segundo ter mo da eq. (3.6).

A transformada de Fouriex do operador entre colchetes da ex pressão (3.16) tem a forma

$$
\overline{\mathrm{W}}_{\mu \nu}(\mathrm{k})=\left(-\delta_{\mu \nu} \cdot \mathrm{k}^{2}+\left(1-\frac{1}{\alpha}\right) \mathrm{k}_{\mu} \mathrm{k}_{\nu}\right) \delta^{\mathrm{ab}}
$$

Para acharmos o inverso deste operador, escrevemos sem per da de generalidades, a equação

$$
\left\{-\delta_{\mu \rho} k^{2}+\left(I-\frac{1}{\alpha}\right) k_{\mu} k_{\nu}\right\}\left\{\delta_{\rho \nu} A+B k_{\rho} k_{\nu}\right\}=\delta_{\mu \nu} \cdot
$$


Resolvendo-a para as incógnitas $A$ e $B$ temos

$$
\left[\tilde{W}_{\mu \nu}(k)\right]^{-1}=-\frac{\delta \mu \nu}{k^{2}}+\frac{(1-\alpha)}{k^{4}} k_{\mu} k_{\nu}
$$

e o propagador como definido em (3.11) tendo a forma

$$
\underset{\mu}{a \sim \sim \sim \sim \sim \sim \Omega^{b}}=D_{\mu \nu}^{a b}(k)=\frac{\delta}{(2 \pi)^{\nu}}\left\{\frac{\delta \mu \nu}{k^{2}}-A \frac{k \mu^{k} \nu}{k^{4}}\right\} \text {, }
$$

onde $A=1-\alpha$.

\section{3 .2 - O PROPAGADOR DO FANTASMA}

E obtido do termo quadrätico nos campos fantasmas da ação efetiva

$$
-i \int d^{\nu} x \partial_{\mu} \bar{c}^{a} \partial_{\mu} c^{b} \delta^{a b}
$$

devemos fazer como foi feito para o propagador do glüon, di ferindo apenas no fato de que os campos de fantasmas são complexos. Portanto temos para o propagador do fantasma

$$
\vec{a}-\vec{k}--\vec{b}=G^{a b}(k)=\frac{-i \delta^{a b}}{(2 \pi)^{v} k^{2}}
$$

\section{3 .3 - O VERTICE DE TRES GLUONS}

E obtido dos termos cübicos nos campos de gau ge da ação efetiva

$$
\int d^{\nu} x q t^{a_{1} a_{2} a_{3}} \delta_{\mu_{1} \mu_{2}} \partial_{\mu_{2}}^{x} A_{\mu_{1}}^{a_{1}}(x) A_{\mu_{2}}^{a_{2}}(x) A_{\mu_{3}}^{a_{3}}(x)
$$


da eq. (3.14)

onde $a_{1}=a, a_{2}=b, a_{3}=c, \mu_{1}=v, \mu_{2}=u, \mu_{3}=p$.

substituindo os campos $\mathrm{A}_{\mu_{i}}^{a_{i}}(\mathrm{x})$ por suas transformadas de Fourjer $a_{\mu_{i}}^{a_{i}}\left(k_{i}\right)$, aplicando o operador $\partial_{\mu_{2}}^{x}$ e integrando em $x$ chegamos $a^{i}$

$$
\begin{aligned}
& -i g t^{a_{1} a_{2} a_{3}} \int d k_{1} d k_{2} d k_{3}(2 \pi)^{v} \delta\left(k_{1}+k_{2}+k_{3}\right) \\
& \delta_{\mu_{1} \mu_{3}} k_{\mu_{2}} a_{\mu_{1}}^{a_{1}\left(k_{1}\right)} a_{\mu_{2}}^{a_{2}\left(k_{2}\right)} a_{\mu_{3}}^{a_{3}\left(k_{3}\right)} \text {. }
\end{aligned}
$$

Daqui obtemos

$$
\alpha_{i_{1} i_{2} i_{3}}\left(k_{1}, k_{2}, k_{3}\right)=-i g t^{a_{1} a_{2} a_{3}} \delta_{\mu_{1} \mu_{3}} k_{\mu_{\mu_{2}}}
$$

onde os indices $i_{1}, i_{2}, i_{3}$ se referem respectivamente aos
termos $\left(\mu_{1}, a_{1}, k_{1}\right),\left(\mu_{2}, a_{2}, k_{2}\right),\left(\mu_{3}, a_{3}, k_{3}\right)$. Como defi nido na seção 3.2 o vértice é dado por

$$
\begin{aligned}
v_{\mu_{1} \mu_{2} \mu_{3}}^{a_{1} a_{2} a_{3}}\left(k_{1}, k_{2}, k_{3}\right)= & -i g(2 \pi)^{\nu} \delta\left(k_{1}+k_{2}+k_{3}\right) \\
& \{1,2,3\}
\end{aligned}
$$

A soma é feita sobre todas as permutações dos elementos do conjunto $\{1,2,3\}$.

Consequentemente temos para aquele, 


$$
\begin{aligned}
& v_{\mu_{1} \mu_{2} \mu_{3}}^{a_{1} a_{2} a_{3}}\left(k_{1}, k_{2}, k_{3}\right)=-i g(2 \pi)^{v} \delta\left(k_{1}+k_{2}+k_{3}\right)
\end{aligned}
$$

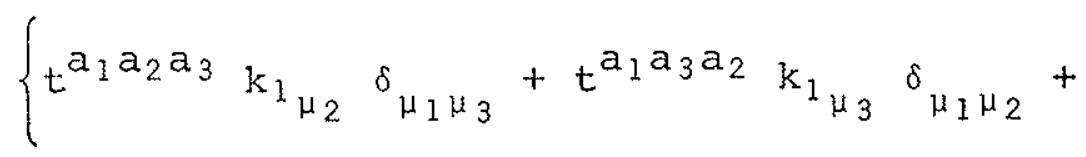

$$
\begin{aligned}
& +t^{a_{2} a_{1} a_{3}} k_{2 \mu_{1}} \delta_{\mu_{2} \mu_{3}}+t^{a_{2} a_{3} a_{1}} k_{2} \mu_{3} \delta_{\mu_{2} \mu_{1}}+ \\
& \left.+t^{a_{3} a_{1} a_{2}} k_{3 \mu_{1}} \delta_{\mu_{3} \mu_{2}}+t^{a_{3} a_{2} a_{1}} k_{3 \mu_{2}} \delta_{\mu_{3} \mu_{1}}\right\}
\end{aligned}
$$

e Einalmente

$$
\begin{aligned}
& z_{3} v^{k_{3}} \quad v_{\mu_{1} \mu_{2} \mu_{3}}^{a_{1} a_{2} a_{3}}\left(k_{1}, k_{2}, k_{3}\right)=-i g(2 \pi)^{\nu} \times
\end{aligned}
$$

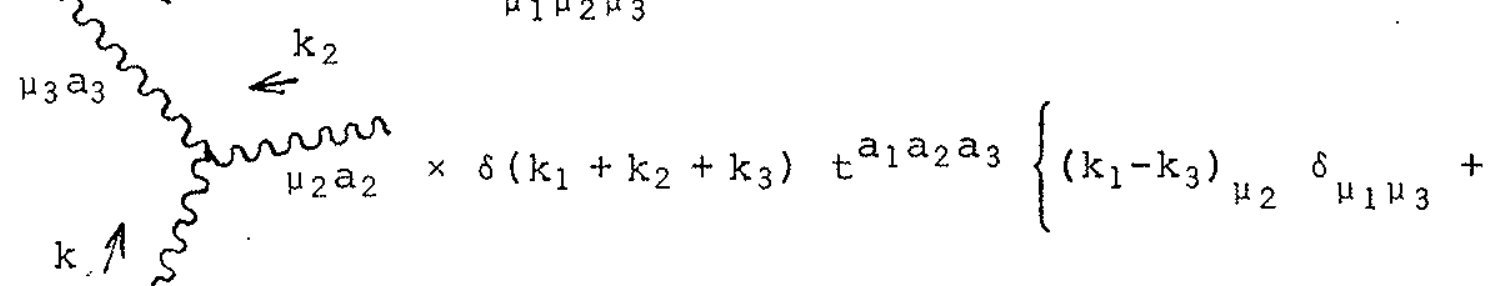

$$
\begin{aligned}
& \left.S^{5} \mu_{1} a_{1}+\left(k_{2}-k_{1}\right)_{\mu_{3}} \delta_{\mu_{1} \mu_{2}}+\left(k_{3}-k_{2}\right)_{\mu_{1}} \delta_{\mu_{2} \mu_{3}}\right\}
\end{aligned}
$$

Depois de feita a simetrização acima a expressão (3.24) fica igual a

$$
\frac{1}{3 !} \int d k_{1} d k_{2} d k_{3} v_{\mu_{1} \mu_{2} \mu_{3}}^{a_{1} a_{2} a_{3}}\left(k_{1}, k_{2}, k_{3}\right) a_{\mu_{1}}^{a_{1}}\left(k_{1}\right) a_{\mu_{2}}^{a_{2}}\left(k_{2}\right) a_{\mu_{3}}^{a_{3}}\left(k_{3}\right) .
$$

o fator 1/3! foi colocado para compensar a soma que foi fej ta. 


\section{$3.3 .4-$ O VERTTCE DE QUATRO GLOONS}

Este vem do termo quädruplo nos campos de gau ge de ação efetiva

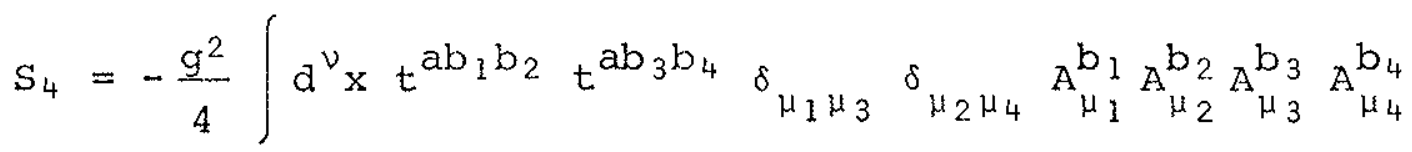

da eq. (3.14),

onde $b_{1}=b, b_{2}=c, b_{3}=a, b_{4}=c$,

$\mu_{1}=\mu, \mu_{2}=v, \mu_{3}=\lambda, \mu_{4}=\rho$;

no espaço de momenta ela fica

$S_{4}=-\frac{g^{2}}{4}(2 \pi)^{\nu} \int a k_{1} \quad d k_{2} \quad d k_{3} d k_{4} t^{a b_{1} b_{2}} t^{a b_{3} b_{4}} \delta_{\mu_{1} \mu_{3}} \delta_{\mu_{2} \mu_{4}} x$

$$
\times a_{\mu_{1}}^{b_{1}}\left(k_{1}\right) \quad a_{\mu_{2}}^{b_{2}}\left(k_{2}\right) \quad a_{\mu_{3}}^{b_{3}}\left(k_{3}\right) \quad a_{\mu_{4}}^{b_{4}}\left(k_{i_{4}}\right)
$$

da expressão acima tiramos o termo $\alpha$ definido em (3.10):

$$
\alpha_{i_{1} i_{2} i_{3} i_{4}}\left(k_{1}, k_{2}, k_{3}, k_{4}\right)=-\frac{g^{2}}{4} t^{a b_{1} b_{2}} t^{a b_{3} b_{4}} \delta_{\mu_{1} \mu_{3}} \delta_{\mu_{2} \mu_{4}},
$$

onde $i_{1}, i_{2}, i_{3}, i_{4}$ se referem respectivamente aos termos:

$$
\left(\mu_{1}, b_{1}, k_{1}\right),\left(\mu_{2}, b_{2}, k_{2}\right),\left(\mu_{3}, b_{3}, k_{3}\right),\left(\mu_{4}, b_{4}, k_{4}\right)
$$

como definido na seção 4.2 , o vëxtice tem a forma 


$$
\begin{aligned}
V_{\mu_{1} \mu_{2} \mu_{3} \mu_{4}}^{a_{1} a_{2} a_{3} a_{4}}\left(k_{1}, k_{2}, k_{3}, k_{4}\right)= & -\frac{g^{2}}{4}(2 \pi)^{\nu} \delta\left(k_{1}+k_{2}+k_{3}+k_{4}\right) \\
& \{1,2,3,4\} t^{a b_{1} b_{2}} t^{a b_{3} b_{4}} \\
& \delta_{\mu_{1} \mu_{3}} \delta_{\mu_{2} \mu_{4}}
\end{aligned}
$$

somando sobre todas as permutações, fazendo algumas manipu lações com indices e reduzindo termos semelhantes temos

$$
v_{\mu_{1} \mu_{2} \mu_{3} \mu_{4}}^{b_{1} b_{2} b_{3} b_{4}}=g^{2}(2 \pi)^{\nu} \delta\left(k_{1}+k_{2}+k_{3}+k_{4}\right)
$$

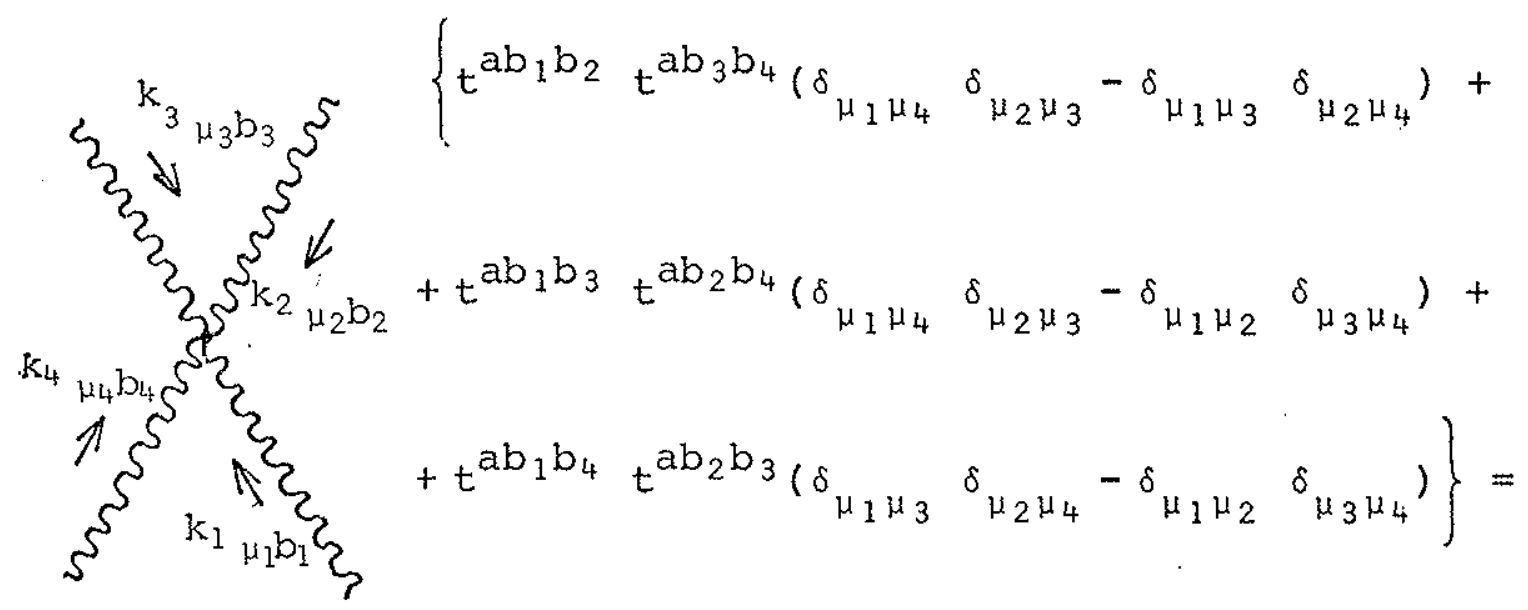

Para complementar, temos que o termo da ação no espaço de momento que gera este vértice tem a forma

$$
\begin{aligned}
& \frac{1}{4 !} \int d k_{1} d k_{2} d k_{3} d k_{4} \underset{\mu_{1} \mu_{2} \mu_{3} \mu_{4}}{\mathrm{~b}_{1} b_{2} b_{3} b_{4}}\left(k_{1}, k_{2}, k_{3}, k_{4}\right) a_{\mu_{1}}^{b_{1}}\left(k_{1}\right) \times \\
& a_{\mu_{2}}^{b_{2}}\left(k_{2}\right) \quad a_{\mu_{3}}^{b_{3}}\left(k_{3}\right) \quad a_{\mu_{4}}^{b_{4}}\left(k_{4}\right)
\end{aligned}
$$




\section{$3.3 .5-$ O VERTICE COM EANTASMAS}

\section{E obtido do termo}

$$
\begin{aligned}
& i \int a x g t^{a b c} \partial_{\mu} \vec{C}^{a} A_{\mu}^{b} c^{c}=i g t^{a b c} \int d k_{1} a k_{2} a k_{3} k_{1} x \\
& \times \delta\left(k_{1}+k_{2}+k_{3}\right)(2 \pi)^{v} \bar{c}^{a}\left(k_{1}\right) a^{b}\left(k_{2}\right) c^{c}\left(k_{3}\right) .
\end{aligned}
$$

Portanto

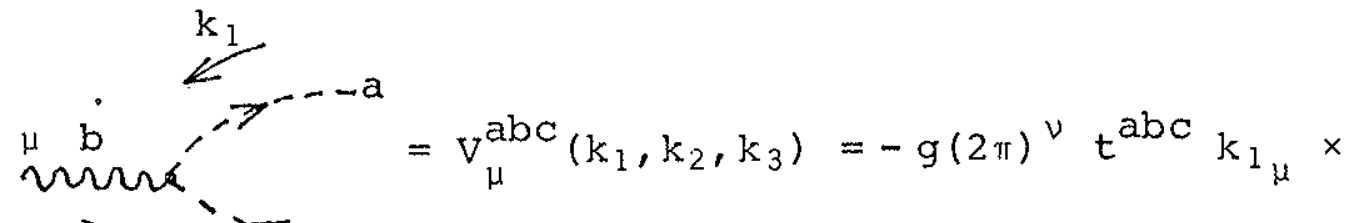

$$
\begin{aligned}
& \rightarrow \mathrm{k}_{2}-\mathrm{k}_{3} \quad \times \delta\left(\mathrm{k}_{1}+\mathrm{k}_{2}+\mathrm{k}_{3}\right)
\end{aligned}
$$

\section{$3.3 .6-$ O VERTICE NO CONTORNO}

E obtido do fator de fase não integrável da eq. (3.13),

$$
\begin{aligned}
\operatorname{ig} \oint_{A_{\mu}(x) d x^{\mu}} & =\operatorname{ig} \oint A_{\mu}^{a}(x) \lambda^{a} d x^{\mu} \\
& =i g \int d k \oint a_{\mu}^{a}(k) \lambda^{a} e^{i k x} d x^{\mu}
\end{aligned}
$$

portanto o vërtice fica

$$
\underset{k}{\operatorname{man}} \operatorname{ig} \oint d x^{\mu} \lambda^{a} e^{i k x}
$$




\section{4 - EATORES COMBINATORIOS}

- cálculo destes fatores para o loop de Wilson, é feito como o da teoria de Yang-Mills para pontos fora do contorno, sendo que os propagadores que ligam a pontos des te, devem ser considerados como pernas externas. Posterior mente deverá haver um fator $1 / \mathrm{n}$ ! onde $\mathrm{n}$ é o número de pon tos na curva. No caso em que o propagador liga dois pontos no contorno, deve-se contar o nümero de possibilidades dele se ligar aos värios pontos do contorno de modos topologica mente distintos. Para se construir diagramas topologicamen te distintos devemos considerar que os vários pontos no con torno são diferentes.

Seja o diagrama

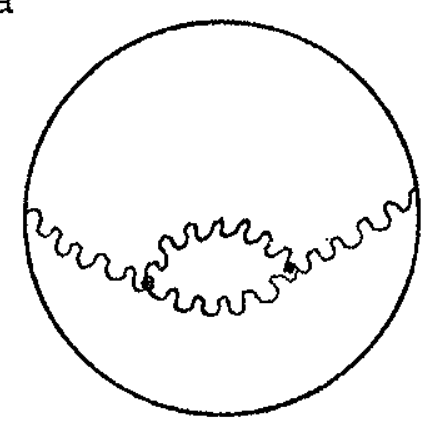

vamos separar os dois vértices fora do loop.
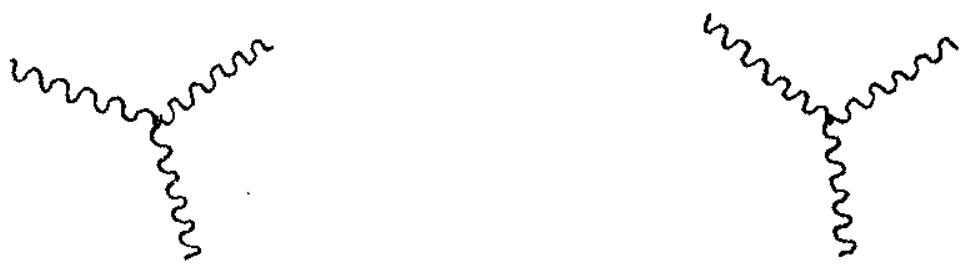

Existem 6 possibilidades para escolher a perna esquerda da quele diagrama; depois do que, com o vërtice que sobra, exis tem três possibilidades para se escolher a perna direita, finalmente existem duas maneiras de se unir as pernas res tantes,

assim

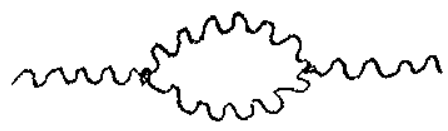

ou assim

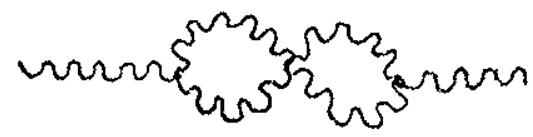


o fator combinatorio será obtido da seguinte maneira: Ini cialmente multiplicamos as possibilidades deduzidas anteri ormente, em seguida deveremos dividir pelo fatorial do nume ro de vértices iguais fora do contorno; dividir pelo fatori al do numero de pontos do contorno; multiplicar pelo coefi ciente do termo na lef (depois de feita a simetrização ex plicada na seção 3.3 .3$)$ que no caso do vértice de três glüons é 1/3!, veja eq. (3.29).

Portanto o fator combinatório fica:

$$
f=\frac{6 \times 3 \times 2}{2 ! 3 ! 3 ! 2 !}=\frac{1}{4}
$$

No caso em que os propagadores estão entre pontos no loop devemos contar o número de possibilidades, con siderando que os pontos são distinguíveis. Tomamos o cuida do de manter a topologia do diagrama.

Ex.: Existem três possibilidades para o diagrama

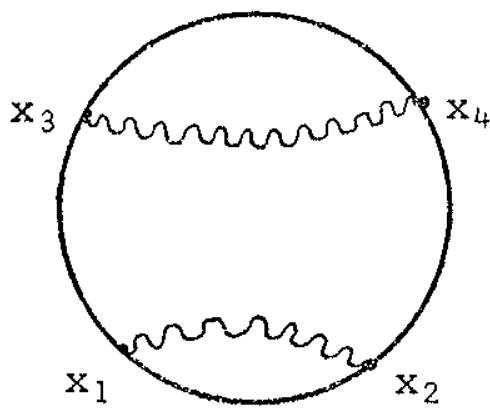

que são:
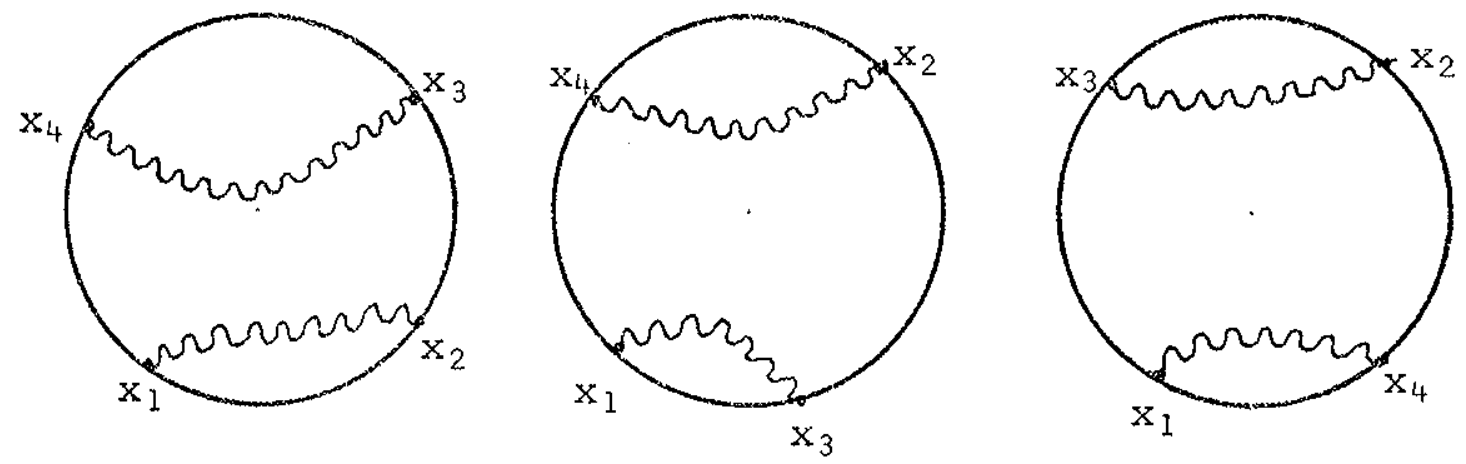
o Eator combinatório, portanto, fica txês dividido pelo fa torial do número de pontos do loop.

\section{5 - EXPRESSOES DOS DIAGRAMAS ATE A 4 a ORDEM}

Convém salientar que para cada loop de fantas ma corresponde um fator (-1); deve-se tomar o traço sobre os geradores do grupo e fazer a ordenação de Dyson ao longo da curva.

Chegamos no ponto de escrever as expressões dos diagramas que sexão calculados do capitulo seguinte.

Em segunda ordem temos:

$\mathrm{G}_{2}=$

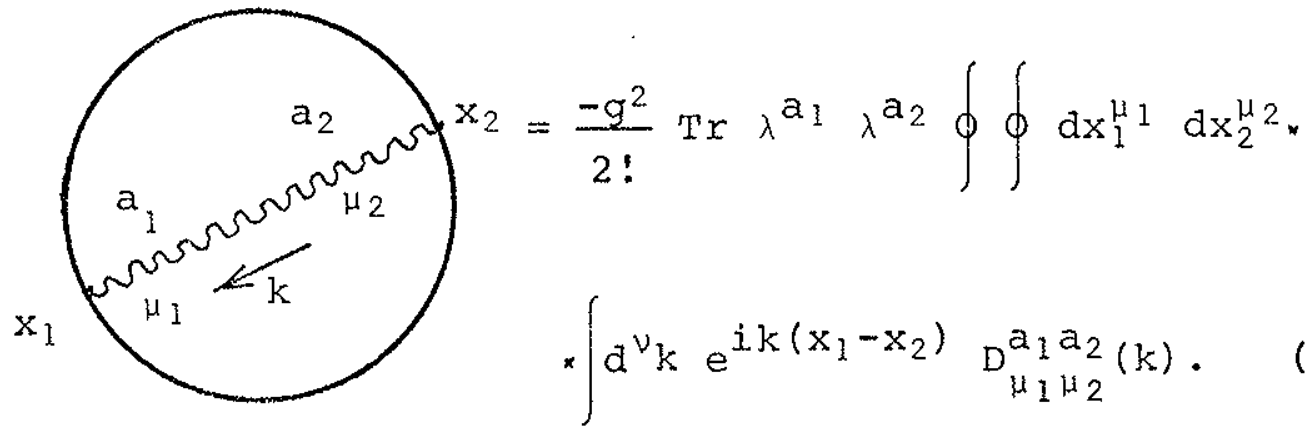

Em quarta ordem temos: 
o vêrtice com três glüons:

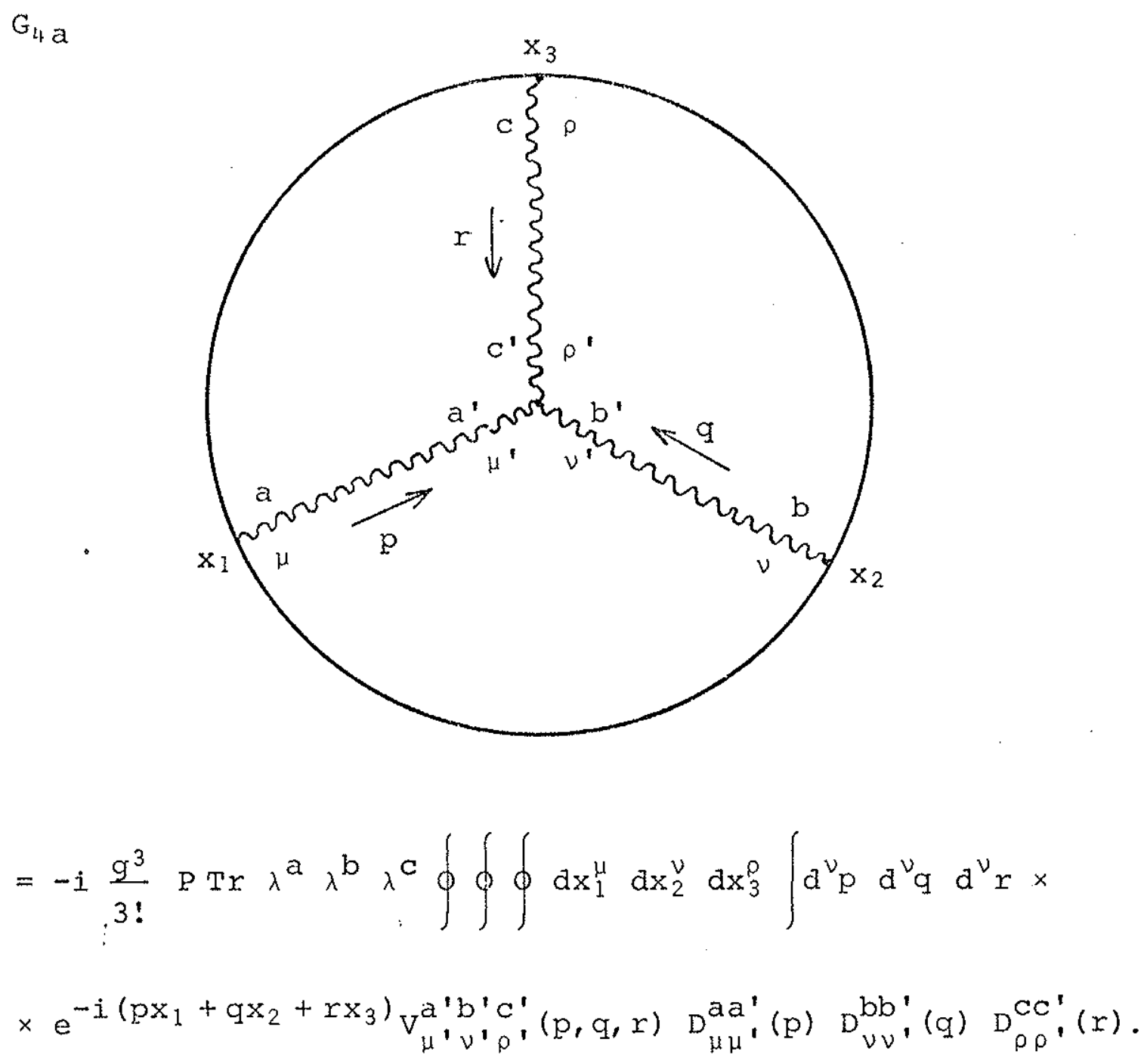

$(3.40)$ 
o gráfico com loop de glúons

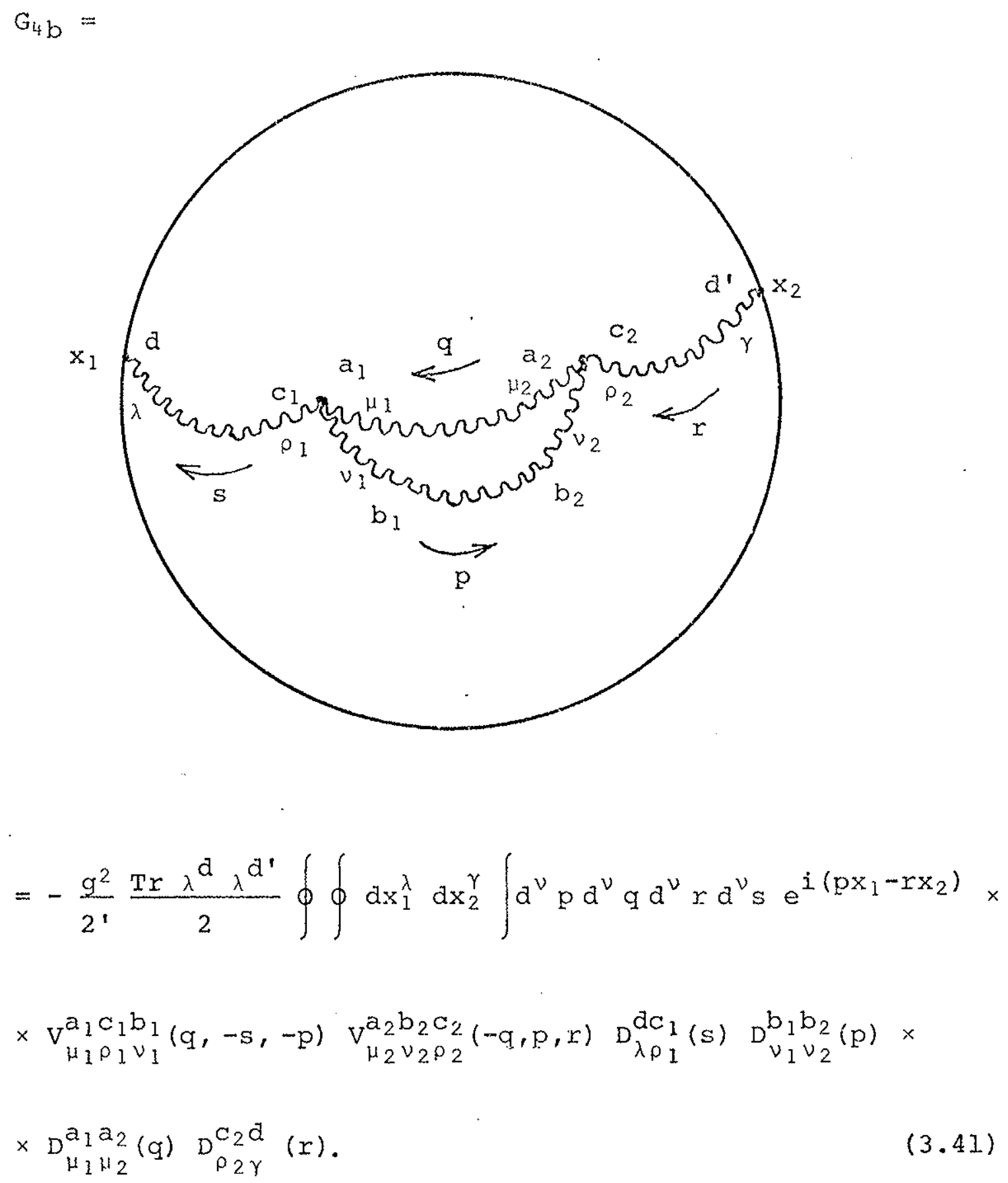


o grâfico com fantasmas

$\mathrm{G}_{4 \mathrm{C}}=$

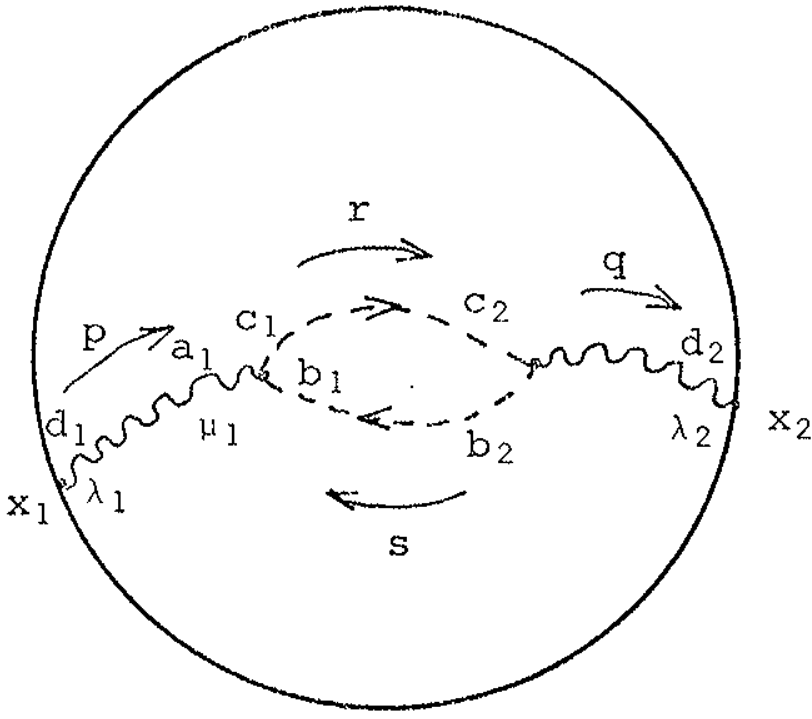

$=+\frac{g^{2}}{2 ! 2} \operatorname{Tr} \lambda^{d_{1}} \lambda^{d_{2}} \oint \oint d x_{1}^{\lambda_{1}} d x_{2}^{\lambda_{2}} \int d^{\nu} p d^{\nu} q a^{\nu} x d^{\nu} s x$

$\times e^{i\left(q x_{2}-p x_{1}\right)} D_{\lambda_{1} \mu_{1}}^{d_{1} a_{1}}(p) D_{\mu_{2} \lambda_{2}}^{a_{2} d_{2}}(q) V_{\mu_{1}}^{c_{1} a_{1} b_{1}}(-r, p, s) \times$

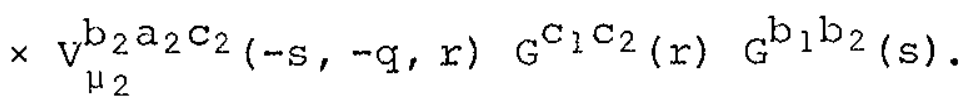


o gráfico do girino com duas pexnas

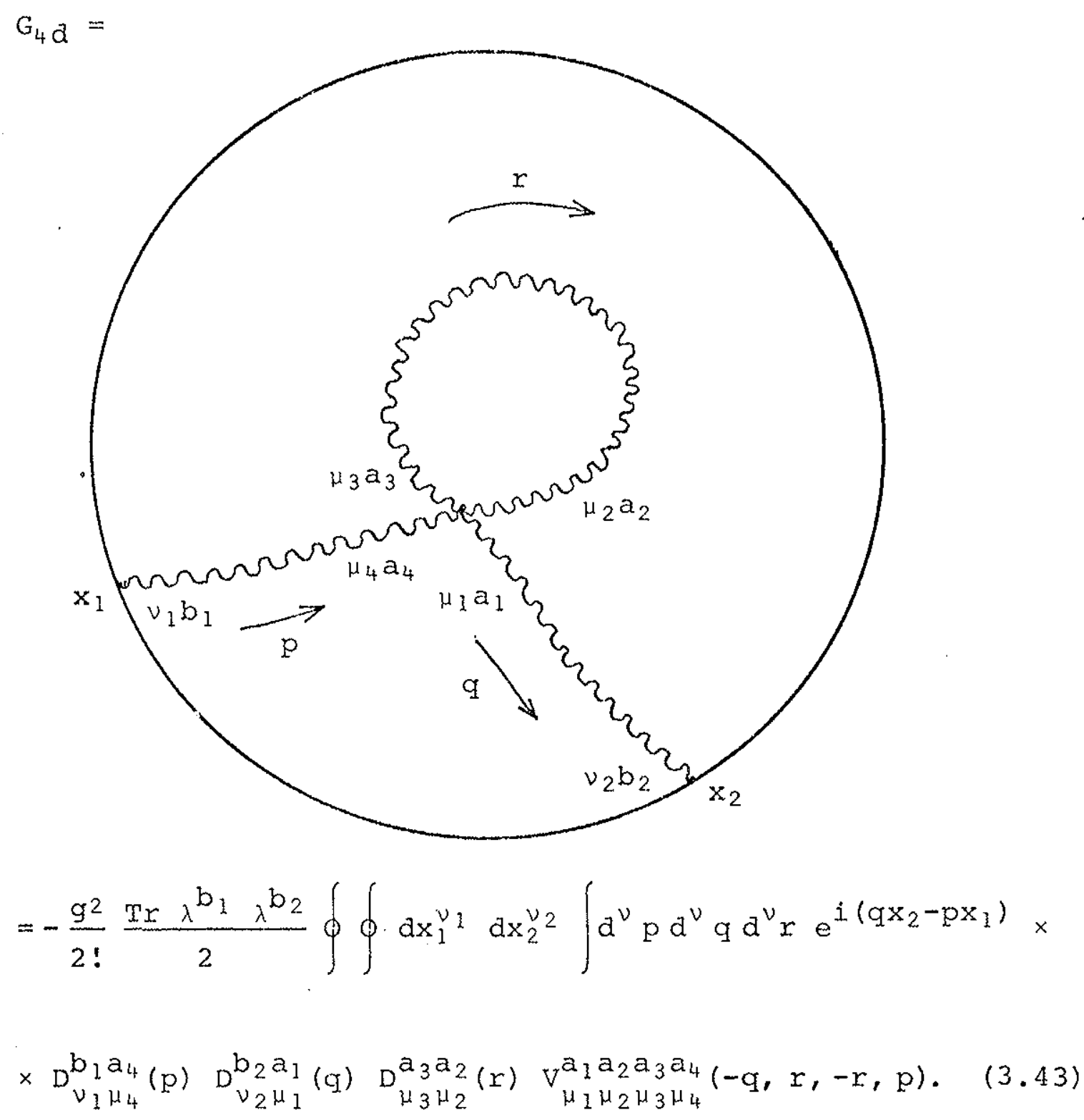


O gráfico do girino com uma perna

$\mathrm{G}_{4 \mathrm{e}}=$

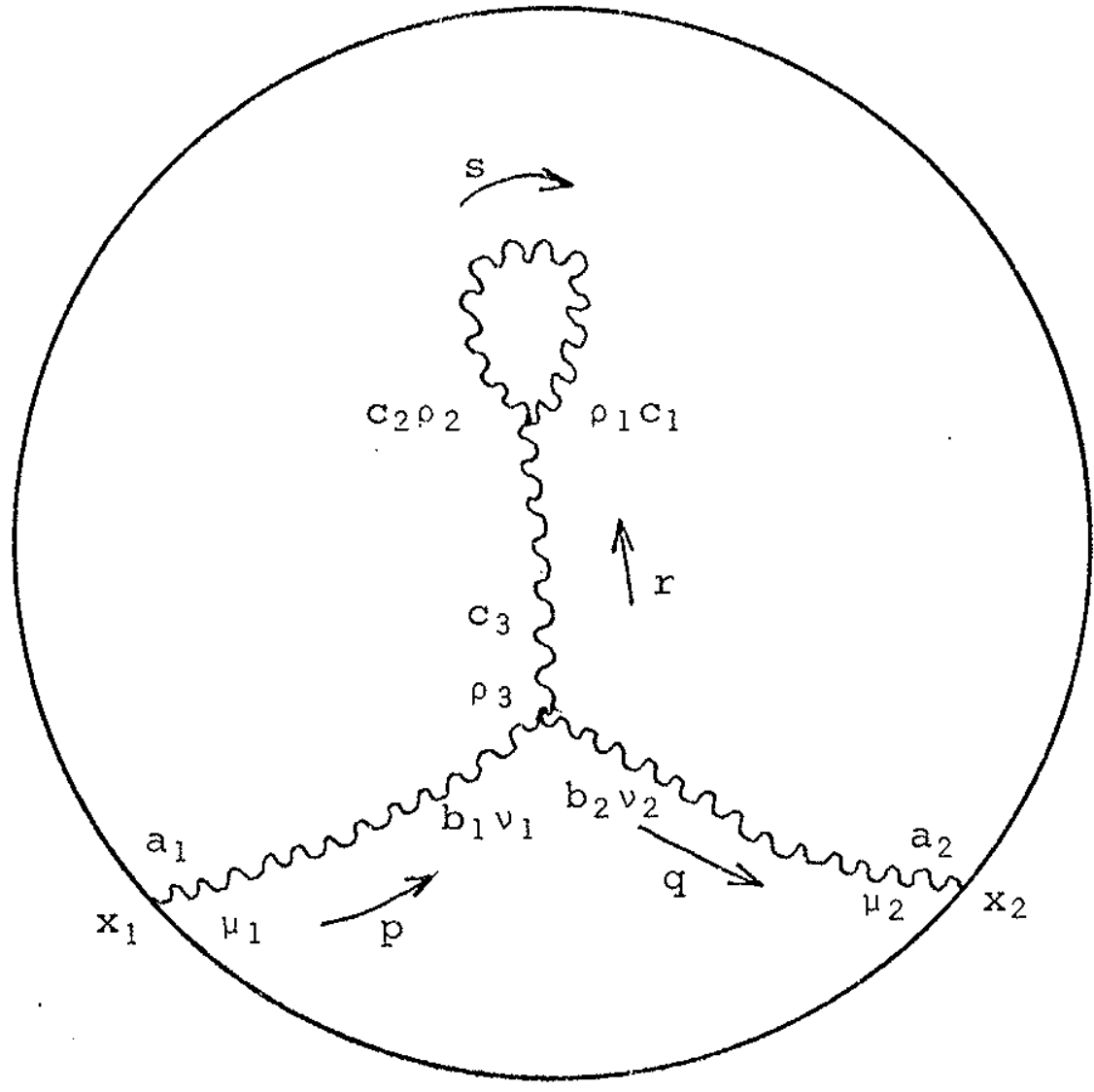

$=-\frac{3}{8} \operatorname{Tr} \lambda^{a_{1}} \lambda^{a_{2}} \oint \oint d x_{1}^{\mu_{1}} d x_{2}^{\mu_{2}} \int d^{\nu} p a^{\nu} q d^{\nu} r d^{\nu} s e^{i\left(q x_{2}-p x_{1}\right)} x$

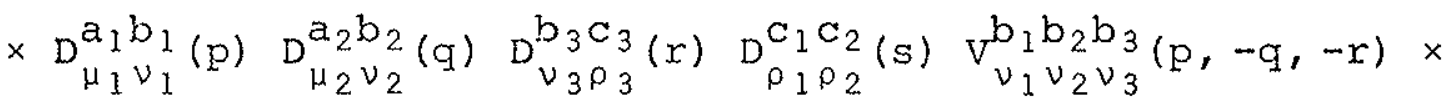

$\times \mathrm{V}_{\rho_{1} \rho_{2} \rho_{3}}^{\mathrm{C}_{1} \mathrm{C}_{2} \mathrm{C}_{3}}(-\mathrm{s}, \mathrm{s}, \mathrm{r})$ 
O grāfico com dois glúons não interagentes.

$$
\mathrm{G}_{4 \mathrm{E}}=
$$

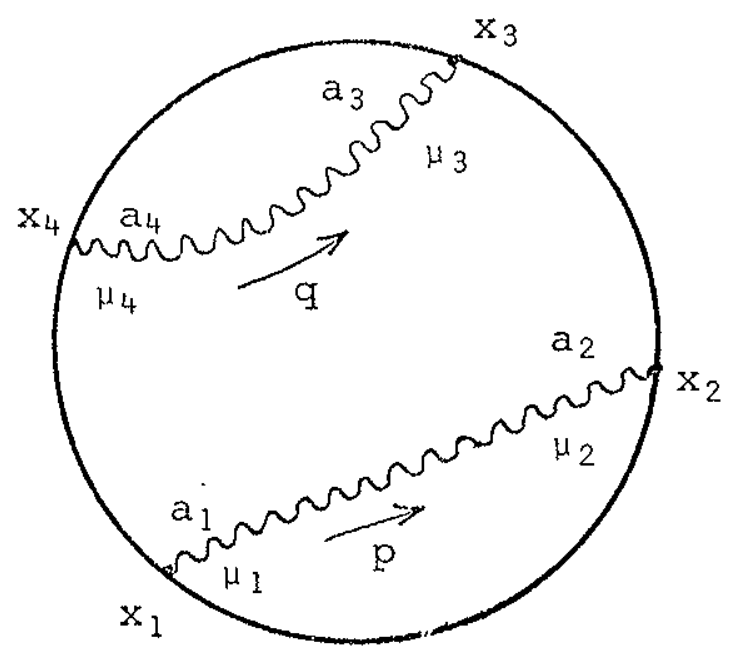

$=\frac{3 g^{4}}{4 !} \mathrm{P} \mathrm{I}^{\mathrm{M}} \mathrm{x} \lambda^{\mathrm{a}_{1}} \lambda^{\mathrm{a}_{2}} \lambda^{\mathrm{a}_{3}} \lambda^{\mathrm{a}_{4}} \oint \oint \oint \oint d x_{1}^{\mu_{1}} d \mathrm{x}_{2}^{\mu_{2}} d x_{3}^{\mu_{3}} d x_{4}^{\mu_{4}} \times$

$$
\times e^{i p\left(x_{2}-x_{1}\right)} \quad e^{i q\left(x_{3}-x_{4}\right)} D_{\mu_{1} \mu_{2}}^{a_{1} a_{2}}(p) D_{\mu_{3} \mu_{4}}^{a_{3} a_{4}}(q)
$$


o gräfico com 2 glüons não interagentes cruza dos.

$$
\mathrm{G}_{49}=
$$

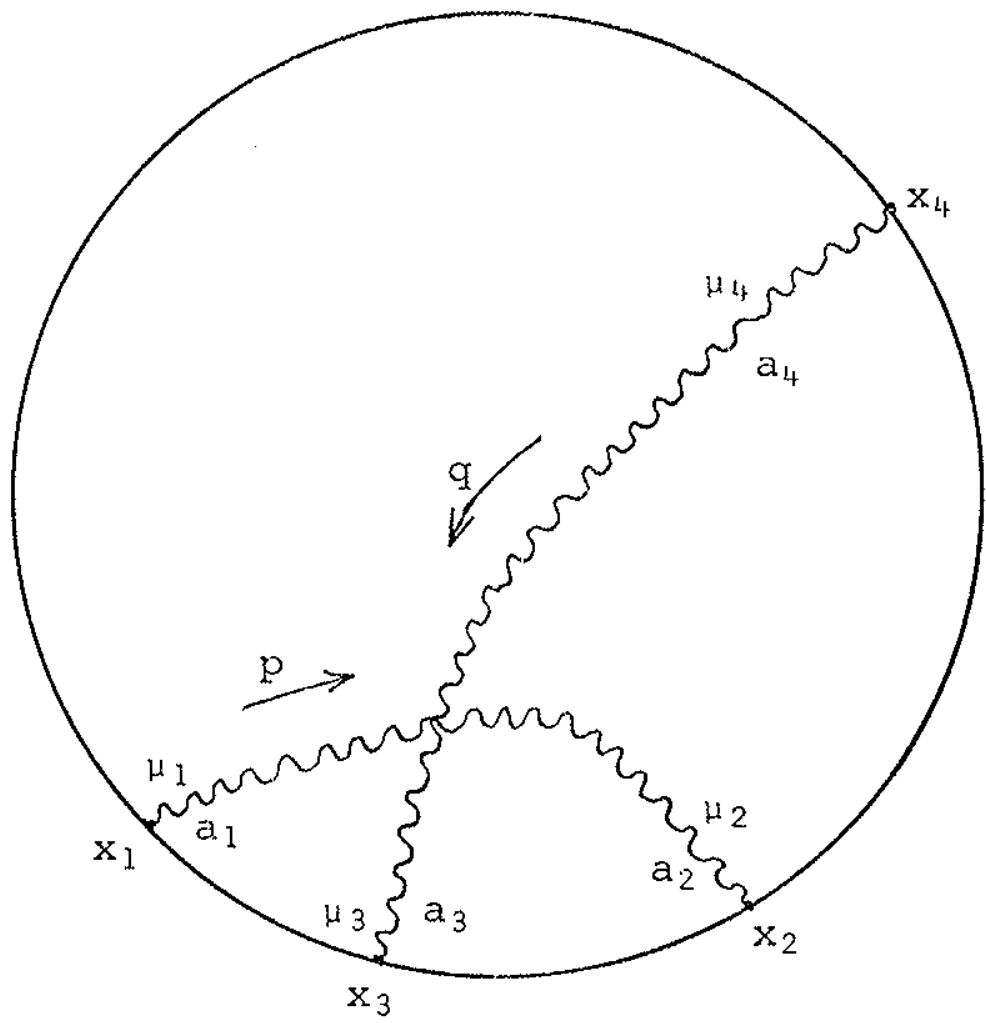

$=\frac{3}{4 !} g^{L_{4}} \operatorname{PTr} \lambda^{a_{1}} \lambda^{a_{3}} \lambda^{a_{2}} \lambda^{a_{4}} \oint \oint \oint \oint d x_{1}^{\mu_{1}} d x_{2}^{\mu_{2}} d x_{3}^{\mu_{3}} d x_{4}^{\mu_{4}} x$

$$
\times e^{i p\left(x_{2}-x_{1}\right)} e^{i q\left(x_{3}-x_{4}\right)} D_{\mu_{1} \mu_{2}}^{a_{1} a_{2}}(p) D_{\mu_{3} \mu_{4}}^{a_{3} a_{4}}(q) .
$$




\section{CALCULOS DOS DIAGRAMAS}

Neste capitulo calculamos as expressões cor respondentes aos diagramas de segunda e quarta ordens. Al guns cälculos, devido a sua extensão, tiveram que ser fei tas com o auxílio do programa schoonschIP. Esses cälculos se encontram nos apêndices $B$ e C. Finalizamos mostrando a expressão da carga renormalizada da cromodinâmica quântica em quarta ordem.

\section{1 - ANALISE DAS DIVERGENCIAS}

Nesta seção apresentamos algumas dificulda des inerentes ao cálculo perturbativo do loop de Wilson. No cálculo do valor esperado no vācuo do fator de fase, ou seja, o loop de Wilson, envolvem integrações no contorno que são divergentes. Esse problema pode ser sanado, como fc. mostrado por Dotsenko e Vergelles [4.1] e Brandt, Neri, sato [4.2]. Aqueles para curvas lisas e estes para curvas que se interceptam as quais podem ter pontas. Essas diver gências que aparecem são removidas com a renormalização.

Quando os vértices no contorno coalescem, co mo está na fig. 4.1, surgem divergências lineares.

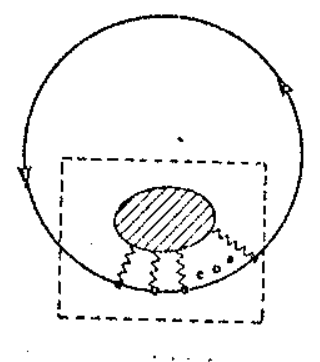

fig. 4.1

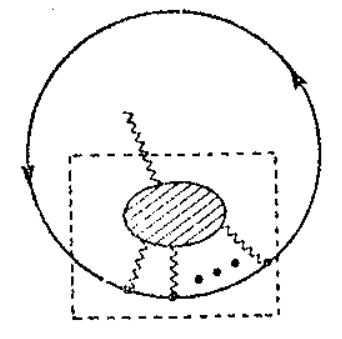

fj.g. 4.2

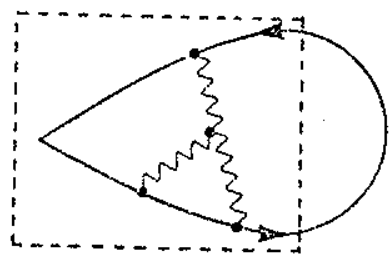

fig. 4.3 
Tajs divergências aparecem em todas as ordens de perturba ção, mas elas não são dificeis de se lidar. Giambiagi, Bollini e Abud [4.3] mostraram, explicitamente, que se usar mos a regularização dimensional, as divergências lineares são automaticamente removiäas. Neste trabalho tambëm não aparecem essas divergências por esse motivo.

Outras divergências aparecem quando os vérti ces, exceto um, coalescem fig. (4.2). Essas divergências são logaritmicas e são xemovidas com a renoxmalização da constante de acoplamento. Neste capítulo, fizemos essa re noxmalização até a quarta ordem na constante de acoplamen to usando a regularização dimensional. Há um outro tipo de divergências que são devidas a existência de possiveis pon tas e interseções na curva C (fig. 4.3). Esse tipo de di vergência continua sendo multiplicativamente renormaliza vel, isto é, existe um $\mathrm{z}(\gamma)$, onde gama é o ângulo formado pelas curvas que dão origem à ponta no ponto de interces são, tal que, $w_{R}\left(C_{\gamma}\right)=z(\gamma) w(C)$. Onde $W_{R}\left(C_{\gamma}\right)$ e o loop de Wilson renormalizado e $Z(\gamma)$ não depende de nenhuma outra característica da curva $C$ que não seja o ângulo $\gamma$.

o câlculo perturbativo do loop de wilson em segunda ordem foj. feito por Giambiagi, Bollini e Abud, que obtiveram resultados finitos em quatro dimensões para cur vas lisas. Em quarta ordem, no entanto, aparecem divergên cias, como já foi dito. A seguir renormalizamos o loop de wilson em quarta ordem usando a regularização dimensional.

\section{2 - CALCULO DOS DIAGRAMAS}

No capitulo anterior deduzimos as regras de Feynman e obtivemos as expressões dos diagramas da teoria até a quarta ordem. 


\section{$4.2 .1-$ DIAGRAMA DE SEGUNDA ORDEM}

Nessa ordem temos a expressão (3.39):

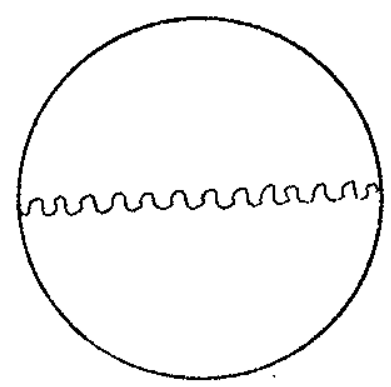

$G_{2}=-\frac{g^{2}}{2 !} \operatorname{Tr}\left(\lambda^{a_{1}} \lambda^{a_{2}}\right) \int d^{\nu} k \oint \oint d x_{1}^{\mu_{1}} d x_{2}^{\mu_{2}} e^{i k\left(x_{1}-x_{2}\right)} D_{\mu_{1} \mu_{2}}^{a_{1} a_{2}(k)}$,

onde $\quad D_{\mu_{1} \mu_{2}}^{a_{1} a_{2}}(k)=\delta^{a_{1} a_{2}} D_{\mu_{1} \mu_{2}}(k)$.

Substituindo (4.2) em (4.1) e usando a propriedade $\operatorname{Tr} \lambda^{\mathrm{a}} \lambda^{\mathrm{b}}=$ $=2 \delta^{a b}$, temos

$$
\begin{aligned}
G_{2} & =-g^{2} n \oint \oint d x_{1}^{\mu_{i}} d x_{2}^{\mu_{2}} \int d^{\nu} k e^{i k\left(x_{1}-x_{2}\right)} D_{\mu_{1} \mu_{2}}(k) \\
& =-g^{2} n \oint \oint d x_{1}^{\mu_{1}} d x_{2}^{\mu_{2}} D_{\mu_{1} \mu_{2}}\left(x_{1}-x_{2}\right),
\end{aligned}
$$

onde

$$
\begin{aligned}
D_{\mu_{1} \mu_{2}}\left(x_{1}-x_{2}\right) & =\int d^{\nu_{k}} e^{i k\left(x_{1}-x_{2}\right)} D_{\mu_{1} \mu_{2}}(k)= \\
& =\int d^{\nu_{k}}\left\{\frac{\delta_{\mu_{1} \mu_{2}}}{k^{2}}-\frac{A k_{\mu_{1}} k_{\mu_{2}}}{k^{4}}\right\} e^{i k\left(x_{1}-x_{2}\right)}
\end{aligned}
$$


ou

$$
\begin{aligned}
D_{\mu_{1} \mu_{2}}\left(x_{1}-x_{2}\right) & =\frac{I^{\prime}\left(\frac{v-2}{2}\right)}{4 \pi \nu / 2}\left\{\delta_{\mu_{1} \mu_{2}}\left|x_{1}-x_{2}\right|^{2-\nu}-\right. \\
& \left.-\frac{A \partial_{\mu_{1}} \partial_{\mu_{2}}^{x_{2}}}{2(\nu-4)}\left|x_{1}-x_{2}\right|^{4-v}\right\}
\end{aligned}
$$

\section{2 .2 - GTRINO COM DUAS PERNAS EM QUARTA ORDEM}

Mostramos, em seguida, que os diagramas dos girinos nesta ordem são nulos. A expressão para o grăfico do gixino com duas pernas $G_{4}$ eq. (3.43), tem a forma

$$
\begin{aligned}
& \text { (andisus } \\
& G_{4} d=-\frac{g^{2}}{2 !} \frac{\operatorname{Tr}\left(\lambda^{b_{1}} \lambda^{b_{2}}\right)}{2} \oint \oint d x_{1}^{\nu_{1}} d x_{2}^{\nu_{2}} \int d^{\nu} p d^{\nu} q d^{\nu} r x \\
& \times e^{i\left(q x_{2}-p x_{1}\right)} D_{v_{1} \mu_{4}}^{b_{1} a_{l_{4}}(p)} D_{\nu_{2} \mu_{1}}^{b_{2} a_{1}}(q) D_{\mu_{3} \mu_{2}}^{a_{3} a_{2}}(x) \times \\
& \times V_{\mu_{1} \mu_{2} \mu_{3} \mu_{4}}^{a_{1} a_{2} a_{3} a_{4_{4}}}(-q, r,-r, p)
\end{aligned}
$$

Da expressão anterior podemos observar que a ünica depen dência em $r$ está no propagador $D_{\mu_{3} \mu_{2}}^{a_{3} a_{2}}(r)$, pois a dependên cia de $x$ que deveria existir na função delta do vértice de quatro glúons desaparece porque o momentum $r$ sai de uma e entra em outra perna dele. Então a dependência em $x$ da in tegração fica 


$$
\int a^{\nu} x\left\{\frac{\delta_{\mu_{3} \mu_{2}}}{k^{2}}-\frac{A}{v} \frac{\delta_{\mu_{3} \mu_{2}}}{k^{2}}\right\}=0
$$

Usamos o fato de que a integral sem massa é nula, vide se ६̧ão 2.5 e as relações (2.14). Portanto $\mathrm{G}_{4} \mathrm{~d}=0$.

\section{2 .3 - CALCULO DO DIAGRAMA DO GIRINO COM UMA PERNA}

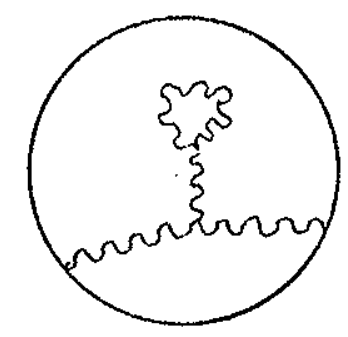

De 3.44 temos

$$
\begin{aligned}
& G_{l_{3}}=-\frac{3 g^{2}}{8} \operatorname{Ir}\left(\lambda^{a_{1}} \lambda^{a_{2}}\right) \oint \oint d x_{1}^{\mu_{1}} d x_{2}^{\mu_{2}} \int d^{\nu} p d^{\nu} q d^{\nu} r d^{\nu} s \\
& \times e^{i\left(q x_{2}-p x_{1}\right)} D_{\mu_{1} \nu_{1}}^{a_{1} b_{1}(p)} D_{\mu_{2} \nu_{2}}^{a_{2} b_{2}}(q) D_{v_{3} \rho_{3}}^{b_{3} c_{3}(x)} D_{\rho_{1} \rho_{2}}^{c_{1} c_{2}}(s) \times \\
& \times v_{v_{1} v_{2} v_{3}}^{b_{1} b_{2} b_{3}}(p,-q,-r) \quad v_{\rho_{1} \rho_{2} \rho_{3}}^{c_{1} c_{2} c_{3}}(-s, s, r)
\end{aligned}
$$

Nesse caso a dependência em $s$ é responsävel pela anulação da integral. O vërtice $\mathrm{V}$ depende linearmente de $s$, portan to temos integrais do tipo

$$
\int a^{\nu} s \frac{s_{\rho_{i}}}{s^{2}} e \int a^{\nu} s \frac{s_{\rho_{i}} s_{\rho_{j}} s_{\rho_{k}}}{s^{4}} .
$$

Ambas as integrais se anulam por simetria, portanto

$$
G_{4 e}=0
$$




\subsection{4 - CAECULO DO DIAGRAMA COM FANTASMAS}

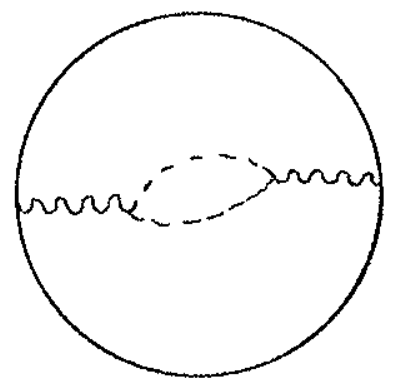

Este é o diagrama $G_{4 C}$ (expressão $(3.42)$ ):

$$
\begin{aligned}
G_{4 c} & =\frac{g^{2}}{2} \operatorname{Tr}\left(\lambda^{a_{1}} \lambda^{a_{2}}\right) \oint \oint d x_{1}^{\lambda_{1}} d x_{2}^{\lambda_{2}} \int d^{\nu} p d^{\nu} q d^{\nu} r d^{\nu} \times \\
& \times e^{\left(q x_{2}-p x_{1}\right)} D_{\lambda_{1} \mu_{1}}^{d_{1} a_{1}}(p) D_{\mu_{2} \lambda_{2}}^{a_{2} d_{2}}(q) v_{\mu_{1}}^{C_{1} a_{1} b_{1}(-r, p, s) \times} \\
& \times v_{\mu_{2}}^{b_{2} a_{2} c_{2}}(-s,-q, r) G^{c_{1} c_{2}}(r) G^{b_{1} b_{2}(s) .}
\end{aligned}
$$

Substituindo os propagadores e os vértices por suas expres sões dadas no Capítulo 3 e utilizando as relações (4.21) te mos

$$
\begin{aligned}
& G_{4 \mathrm{C}}=n g C_{2} \oint \oint d x_{1}^{\lambda_{1}} d x_{2}^{\lambda_{2}} \int d^{\nu} p d^{\nu} q d^{\nu} x d^{\nu} s e^{i\left(q x_{2}-p x_{1}\right)} \times \\
& \times \frac{I}{(2 \pi)^{\nu}}\left\{\frac{\delta \lambda_{1} \mu_{1}}{p^{2}}-\frac{A p_{\lambda_{1}} p_{\mu_{1}}}{p^{4}}\right\} \frac{1}{(2 \pi)^{v}}\left\{\frac{\delta_{\mu_{2} \mu_{2}}}{q^{2}}-\frac{A q_{\mu_{2}} q_{\lambda_{2}}}{q^{4}}\right\} \times \\
& \times(-1) g(2 \pi)^{\nu} r_{\mu_{1}} \delta(-x+p+s)(-1) g(2 \pi)^{\nu} s_{\mu_{2}} \delta(-s-q+r) \times \\
& \times \frac{(-i)}{(2 \pi)^{\nu} r^{2}} \frac{(-i)}{(2 \pi)^{\nu} s^{2}}
\end{aligned}
$$


Muitipjicando os termos e eliminando aqueles que contêm $p_{\lambda_{1}}$ ou $q_{\lambda_{2}}$, una vez que

$$
\oint d x_{1}^{\lambda_{1}} p_{\lambda_{1}} e^{-i p x_{1}}=0 e \oint d x_{2} q_{\lambda_{2}} e^{j q x_{2}}=0
$$

Com isso obtemos,

$$
\begin{aligned}
G_{4 c} & =\frac{g^{4} n C_{2}}{(2 \pi)^{2 v}} \oint \oint d x_{1}^{\lambda_{1}} d x_{2}^{\lambda_{2}} \int \frac{d^{\nu} p d^{\nu} q d^{\nu} r d^{\nu} s}{p^{2} q^{2} r^{2} s^{2}} \times \\
& \times \delta(p+s-x) \delta(x-s-q) r_{\lambda_{1}} s_{\lambda_{2}} e^{-i p x_{1}+i q x_{2}} .
\end{aligned}
$$

Integrando em $x$ temos

$$
\begin{aligned}
& G_{4 C}=\frac{g^{4_{4}} \mathrm{nC}_{2}}{(2 \pi)^{2 \nu}} \oint \oint d x_{1}^{\lambda_{1}} d \mathrm{dx}_{2}^{\lambda_{2}} \times \\
& \times \int \frac{a^{\nu} p a^{\nu} q a^{\nu} s s_{\lambda_{2}}(p+s)_{\lambda_{1}} e^{-i p x_{1}+i q x_{2}} \delta(p-q)}{p^{2} q^{2} s^{2}(p+s)^{2}} .
\end{aligned}
$$

Integrando em $q$ e em s, usando a förmula 3 do apêndice A re sulta

$$
\begin{aligned}
G_{4 c} & =-\frac{n g^{4} C_{2}}{(2 \pi)^{2 \nu}} \oint \oint d x_{1}^{\lambda_{1}} d x_{2}^{\lambda_{2}} \int d^{\nu} p \frac{e^{i p\left(x_{2}-x_{1}\right)}}{p^{4}} \times \\
& \times \frac{\pi^{\nu / 2} \delta_{\lambda_{1} \lambda_{2}}\left(p^{2}\right)^{\frac{\nu}{2}-1} \Gamma\left(2-\frac{v}{2}\right) \Gamma\left(\frac{\nu}{2}-1\right) \Gamma\left(\frac{\nu}{2}-1\right)}{4 \Gamma(\nu-2)(\nu-1)} .
\end{aligned}
$$


Finalmente, integrando em $p$, usando a integral 8 do apêndi. ce $A$, segue

$$
\begin{aligned}
G_{4 C} & =\frac{n g^{4} C_{2} \Gamma\left(2-\frac{\nu}{2}\right) \Gamma\left(\frac{\nu}{2}-1\right) \Gamma\left(\frac{\nu}{2}-1\right) \Gamma(\nu-3)}{2^{8}{ }^{\nu} \nu(\nu-1) \Gamma\left(\frac{6-\nu}{2}\right) \Gamma(\nu-2)} \times \\
& \times \oint \oint d x_{1}^{\lambda_{1}} \mathrm{dx}_{2}^{\lambda_{2}} \frac{\delta_{\lambda_{1} \lambda_{2}}}{\left|\mathrm{x}_{2}-\mathrm{x}_{1}\right|^{2 \nu-6}} .
\end{aligned}
$$

\section{2 .5 - CAICULO DOS DIAGRAMAS DE DOIS GLUONS NÃO INTERAGEN TES}

Esses são os diagramas $G_{4 f} e G_{t_{g}}$ cujas ex pressöes säo respectivamente $(3.45)$ e (3.46), reescritas a seguix:
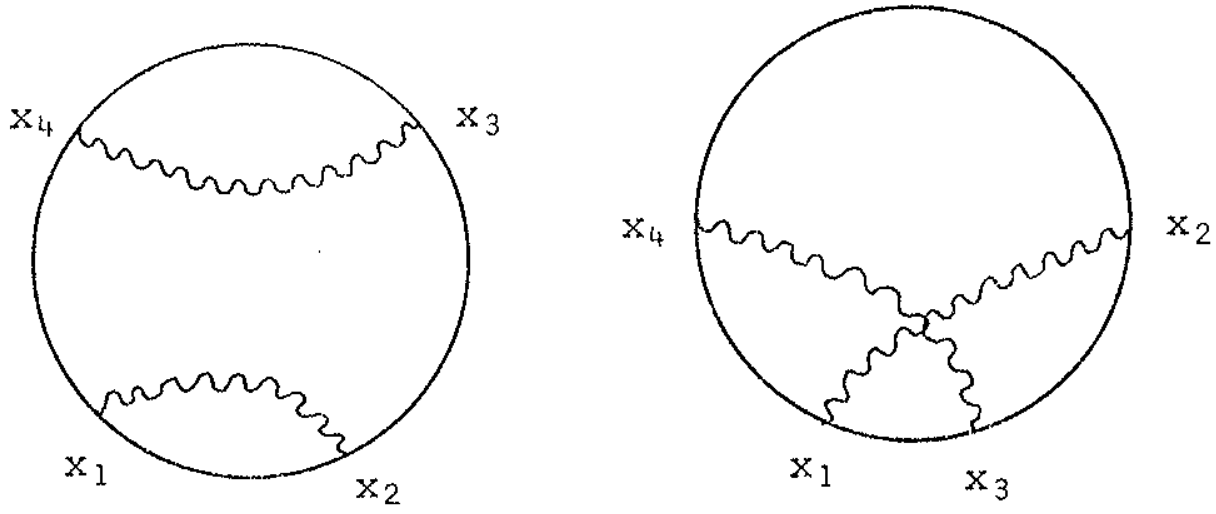

ou

$$
\begin{aligned}
G_{4 f}+G_{4 g} & =\frac{3}{4 !} g^{4}\left(\operatorname{Tr}\left(\lambda^{a_{1}} \lambda^{a_{2}} \lambda^{a_{3}} \lambda^{a_{4}}\right)+\right. \\
& \left.+\operatorname{Tr}\left(\lambda^{a_{1}} \lambda^{a_{3}} \lambda^{a_{2}} \lambda^{a_{4}}\right)\right) \delta^{a_{1} a_{2}} \delta^{a_{3} a_{4}} I,
\end{aligned}
$$


onde

$$
\begin{aligned}
I & =p \oint \oint \phi \oint d x_{1}^{\mu_{1}} d x_{2}^{\mu_{2}} d x_{3}^{\mu_{3}} d x_{4_{4}}^{\mu_{4}} D_{\mu_{1} \mu_{2}}\left(x_{1}-x_{2}\right) \times \\
& \times D_{\mu_{3} \mu_{4}}\left(x_{3}-x_{4}\right) .
\end{aligned}
$$

Seja C o coeficiente de I da expressão (4.17). Contraindo os indices internos temos

$\left.C=\frac{3}{4 !} g^{4_{4}}\left(\operatorname{Ir} \lambda^{a_{1}} \lambda^{a_{1}} \lambda^{a_{3}} \lambda^{a_{3}}\right)+\operatorname{Tr}\left(\lambda^{a_{1}} \lambda^{a_{3}} \lambda^{a_{1}} \lambda^{a_{3}}\right)\right)$.

Subtraindo e somando $\operatorname{Tr}\left(\lambda^{a_{1}} \lambda^{a_{1}} \lambda^{a_{3}} \lambda^{a_{3}}\right)$ entre os parênte ses da equação (4.19), podemos escrever

$C=\frac{3}{4 !} g^{4}\left(2 \operatorname{Tr}\left(\lambda^{a_{1}} \lambda^{a_{1}} \lambda^{a_{3}} \lambda^{a_{3}}\right)+\operatorname{Tr}\left(\lambda^{a_{1}}\left[\lambda^{a_{3}}, \lambda^{a_{1}}\right] \lambda^{a_{3}}\right)\right)$.

Com as relações,

$$
\begin{aligned}
& \operatorname{Tr}\left(\lambda^{a} \lambda^{b}\right)=2 \delta^{a b}, \\
& t^{a b c} \operatorname{Tr}\left(\lambda^{a} \lambda^{b} \lambda^{c}\right)=2 i n c_{2}, \\
& {\left[\lambda^{a}, \lambda^{b}\right]=i t^{a b c} \lambda^{c},} \\
& \left\{\lambda^{a}, \lambda^{b}\right\}=i d^{a b c} \lambda^{c}, \\
& t^{a b c} t^{a b a}=c_{2} \delta^{c d},
\end{aligned}
$$

obtemos 


$$
C=\frac{3}{4 !} 9^{4}\left(2 \times 4 n-4 n C_{2}\right)
$$

onde $\mathrm{C}_{2} \ddot{\mathrm{e}}$ a constante de casimir quadxatica. Substituindo $C$ em $(4.17)$, obtemos

$$
\begin{aligned}
G_{4 f}+G_{4 g} & =n\left[-g^{2} \oint \oint d x_{1}^{\mu} d x_{2}^{\nu} D_{\mu \nu}\left(x_{1}-x_{2}\right)\right]^{2}- \\
& -\frac{n g^{4} C_{2}}{2} \oint \oint d x_{1}^{\mu} d x_{2}^{\mu_{2}} D_{\mu_{1} \mu_{2}}\left(x_{1}-x_{2}\right) x \\
& \times \int_{x_{1}}^{x_{2}} d x_{3}^{\mu} \int_{x_{2}}^{x_{1}} d x_{4}^{\mu_{4}} D_{\mu_{3} \mu_{4}}\left(x_{3}-x_{4}\right) .
\end{aligned}
$$

Neste ponto devemos obsexvar que o primeiro termo do lado direito da expressão (4.23a) é a parte abeliana da teoria. Somando todos esses termos abejianos que apare cem em todas as oxdens temos

$$
n e^{-g^{2}} \oint d x_{1}^{\mu} 1 \oint d x_{2}^{\mu_{2}} D_{\mu_{1} \mu_{2}}\left(x_{1}-x_{2}\right)
$$

que ë a expressão exata para a média do Joop de Wilson nas teorias abelianas.

A expressão (4.23b) apresenta apenas divergên cias Iineares, no caso de curvas lisas, que são eliminadas pela regularização dimensional, portanto esse termo não $e$ levado em conta na renormalização da carga que tem origem nas divergências logaxitmicas. 
Em ordens superiores tambēm aparecem termos que são produtos das expressões dos diagramas irredutĩveis e conexos de ordem inferior, (a conexão é ao longo da cux va). De um modo geral [4.1], temos

$$
<\mathrm{Tx} W(\mathrm{C})>=\mathrm{e}^{\sum(\mathrm{C})} \text {. }
$$

onde $\Sigma(C)$ e a soma sobre todos os diagramas conexos. Por tanto para a renormalização só nos interessa os diagramas conexos.

Para renormalizarmos a carga em quarta ordem, que é a finalidade deste capitulo, temos que escrever os diagramas dessa ordem em termos do de segunda. Devemos, en tão, integrax o segundo termo do lado direito da equação (4.23a) en $x_{3}$ e $x_{4}$. De um modo geral não podemos integrax em $x_{3}$ e $x_{4}$ por causa da dependência dessa integral com a curva. Como serä provado no capitulo 6 , a parte divergente dessa integral não depende nem do arco nem da curva em qua tro dimensões, sendo isso o sufjciente para a renormaliza §ão. Em seguida calculamos a integral

$$
\begin{aligned}
G_{4 g}= & -\frac{n C^{2} g^{4}}{2} \oint d x_{1}^{\mu_{1}} \oint d x_{2}^{\mu_{2}} D_{\mu_{1} \mu_{2}}\left(x_{1}-x_{2}\right) \times \\
& \times \int_{x_{1}}^{x_{2}} d x_{3}^{\mu_{3}} \int_{x_{2}}^{x_{2}} d x_{4}^{\mu_{4}} D_{\mu_{3} \mu_{4}}\left(x_{3}-x_{4}\right) .
\end{aligned}
$$

Para tanto necessitaremos do propagador no espaço de coor denadas que ë dado por 


$$
D_{\mu \nu}(x-y)=\frac{\Gamma\left(\frac{\nu-2}{2}\right)}{4 \pi / 2}\left\{\delta_{\mu \nu}|x-y|^{2-\nu}-\frac{A \partial x \partial y|x-y|^{4}-\nu}{2(\nu-4)}\right\} .
$$

Substituindo essa expressão na integral

$$
I=\int_{x_{1}}^{x_{2}} \mathrm{dx}_{3}^{\mu_{3}} \int_{x_{2}}^{x_{1}} \mathrm{dx}_{4}^{\mu_{4}} \mathrm{D}_{\mu_{3} \mu_{4}}\left(\mathrm{x}_{3}-\mathrm{x}_{4}\right)
$$

temos

$$
\begin{aligned}
I & =\int_{x_{1}}^{x_{2}} d x_{3}^{\mu_{3}} \int_{x_{2}}^{x_{1}} d x_{4}^{\mu_{4}} \frac{I^{\prime}\left(\frac{\nu-2}{2}\right)}{4 \pi^{\nu / 2}}\left\{\delta_{\mu_{3} \mu_{4}}\left|x_{3}-x_{4}\right|^{2-\nu}-\right. \\
& \left.-\frac{A \partial_{\mu_{3}}^{x_{3}} \partial_{\mu_{4}} x_{4}\left|x_{3}-x_{4}\right|^{4-\nu}}{2(\nu-4)}\right\} .
\end{aligned}
$$

o termo que contém $A$ é uma diferencial exata pois é do ti po

$$
\oint d x^{\alpha} \partial_{\alpha} f(x)
$$

Fazendo a parametrização

$$
\begin{array}{ll}
x^{\alpha}=x^{\alpha}(\sigma), & 0 \leqslant 0 \leqslant 2 \pi \\
d x^{\alpha}=\frac{d x^{\alpha}(\sigma)}{d \sigma} d \sigma, &
\end{array}
$$


obtemos

$$
\begin{aligned}
\oint d x^{\alpha} \partial_{\alpha} f(x) & =\int_{0}^{2 \pi} d \sigma \frac{d f(x)}{d x^{\alpha}} \frac{d x^{\alpha}}{d \sigma}=\int_{0}^{2 \pi} d \sigma \frac{d f(x)}{d \sigma}= \\
& =f(x(2 \pi))-f(x(0))
\end{aligned}
$$

Portanto podemos escrever

$$
\begin{gathered}
\frac{\Gamma\left(\nu-\frac{2}{2}\right)}{\frac{\Gamma(v / 2}{v / 2} \int_{x_{1}}^{x_{2}} d x_{3}^{\mu_{3}}} \int_{x_{2}}^{x_{1}} d x_{4_{4}}^{\mu_{4}} \frac{A}{2(\nu-4)} \partial_{\mu_{3}}^{x_{3}} \partial_{\mu_{4}}^{x_{4}}\left|x_{3}-x_{4}\right|^{l_{4}-\nu}= \\
=-\frac{A \Gamma\left(\frac{v-2}{2}\right)\left|x_{2}-x_{1}\right|^{4-\nu}}{4 \pi / 2(\nu-4)} .
\end{gathered}
$$

O outro termo da integral I é

$$
\text { I. }=\frac{1\left(\frac{\nu-2}{2}\right)}{4 \pi \nu / 2} \delta_{\mu_{3} \mu_{4}} \int_{x_{1}}^{x_{2}} d x_{3}^{\mu_{3}} \int_{x_{2}}^{x_{1}} d x_{4}^{\mu_{4}}\left|x_{3}-x_{4}\right|^{2-\nu} .
$$

Como será mostrado no capitulo 5 a parte di vergente dessa integral não depende nem do arco nem da cur var, por esse motivo, portanto, podemos integrar sobre uma ciruferencia sem perda de generalidade. Fazendo a mudan ça de variäveis

$$
\begin{aligned}
& x_{3}^{1}=\operatorname{Rcos}\left(\psi_{1}\right), \\
& x_{3}^{2}=\operatorname{Rsen}\left(\psi_{1}\right), \\
& x_{4}^{1}=\operatorname{Rcos}\left(\psi_{2}\right), \\
& x_{4_{4}}^{2}=\operatorname{Rsen}\left(\psi_{2}\right),
\end{aligned}
$$


onde $\mathrm{R}$ é o raio da circunferência. Os ângulos $\psi_{1}$ e $\psi_{2}$ são medidos a partir do eixo (1), vide fig. 4.4, para os pon tos $x_{3} e x_{4}$ respectivamente.

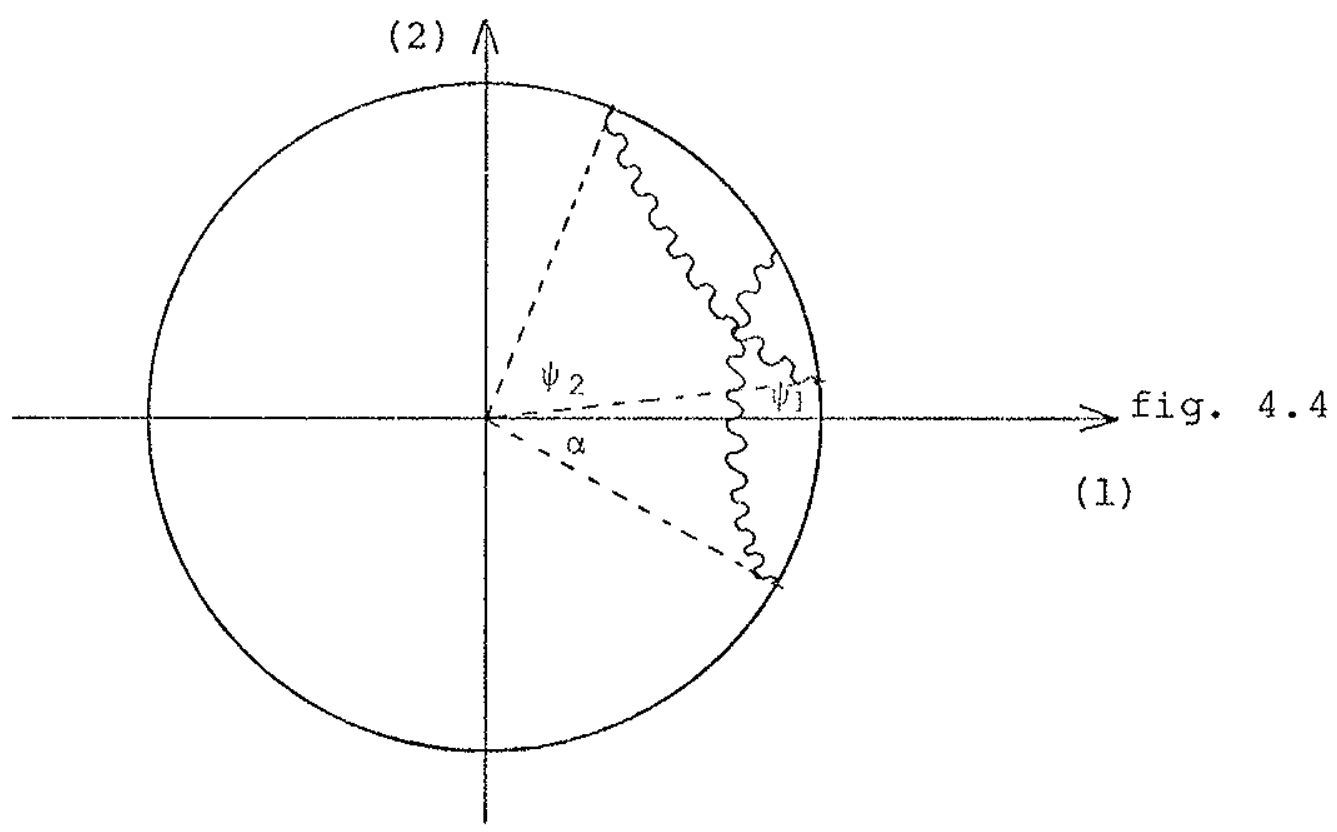

Substituindo as relações (4.35) na equação (4.34), temos

$I^{\prime}=-\frac{\Gamma\left(\frac{\nu-2}{2}\right) 2^{2-\nu} R^{4-\nu}}{8 \pi^{\nu / 2}}\left\{\int_{\alpha}^{2 \pi-\alpha} d \psi_{2} \times\right.$

$\left.\times\left[\int_{-\alpha}^{\alpha} d \psi_{1}\left[\operatorname{sen}^{2}\left(\frac{\psi_{1}-\psi_{2}}{2}\right)\right]^{\frac{2-\nu}{2}}-2 \int_{-\alpha}^{\alpha} d \psi_{1}\left[\operatorname{sen}^{2}\left(\frac{\psi_{1}-\psi_{2}}{2}\right)\right]^{\frac{4-\nu}{2}}\right]\right\}$.

$(4.36)$

onde a é a metade do ângulo subentendido pelo arco entre $\mathrm{x}_{1}$ e $\mathrm{x}_{2}$. 0 eixo (1) pode sempre ser colocado simetricamen te com relação a $x_{1} e x_{2}$, com a finalidade de facilitax os cálculos. No capítulo seguinte mostramos que o termo da equação (4.36) que gera um polo em $v=4$ è aquele cujo expoente $\vec{e} \frac{\nu-2}{2}$. Seja I" a j.ntegral (4.36) sem o segundo termo do 
lado direito. Este temo nos dá um resultado finito, sendo portanto desnecessáxio considerá-lo. Então, temos

$X^{\prime \prime}=-\frac{\Gamma\left(\frac{\nu-2}{2}\right) 2^{2-\nu} R^{4-\nu}}{8 \pi^{\nu / 2}} \int_{\alpha}^{2 \pi-\alpha} d \psi_{1}\left[\operatorname{sen}^{2}\left(\frac{\psi_{1}-\psi_{2}}{2}\right)\right]^{\frac{2-\nu}{2}}$.

ESetuando a mudança de variävel

$x=\operatorname{sen}^{2}\left(\frac{\psi_{1}-\psi_{2}}{2}\right) \cdot d x=\{ \pm\} \sqrt{x^{\prime}} \sqrt{1-x} d \psi_{1}\left\{\begin{array}{l}\psi_{1}>\psi_{2} \\ \psi_{1}<\psi_{2}\end{array}\right\}$.

Integrando em $\psi_{1}$ temos

$$
\begin{aligned}
I^{\prime \prime} & =\frac{\Gamma\left(\frac{\nu-2}{2}\right) 2^{2-\nu} R^{4-\nu}}{8 \pi^{\nu / 2}} \int_{\alpha}^{2 \pi-\nu} d \psi_{2}\left\{\int_{0}^{\operatorname{sen}^{2}\left(\frac{\alpha-\psi_{2}}{2}\right)} d x \frac{\frac{1-\nu}{2}}{\sqrt{1-x}}+\right. \\
& \left.+\int_{0}^{\cdot \operatorname{sen}^{2}\left(\frac{\alpha-\psi_{2}}{2}\right)} d x \frac{\frac{x^{\frac{1-\nu}{2}}}{\sqrt{I-x}}}{}\right\} .
\end{aligned}
$$

Integrando em $x$ e em seguida mudando a variāvel

$$
\begin{aligned}
& y=\operatorname{sen}^{2}\left(\frac{\psi_{2}-\alpha}{2}\right), \\
& d y=\{ \pm\} \sqrt{y} \sqrt{1-y} \text { d } 2\left\{\begin{array}{l}
\psi_{2}-\alpha<\pi \\
\psi_{2}-\alpha>\pi
\end{array}\right\}
\end{aligned}
$$

en uma delas e 


$$
\begin{aligned}
& y=\operatorname{sen}^{2}\left(\frac{\psi 2+\alpha}{2}\right) \\
& d y=\{ \pm\} \sqrt{\mathrm{y}} \sqrt{1-\mathrm{Y}}\left\{\begin{array}{r}
\psi_{2}+\alpha>\pi \\
2 \pi>\psi_{2}+\alpha>\pi
\end{array}\right\}
\end{aligned}
$$

na outra. Temos

$$
\begin{aligned}
& I^{\prime \prime}=-\frac{\Gamma\left(\frac{\nu-2}{2}\right) 2^{2-\nu} R^{4-\nu} 4}{8 \pi \nu / 2}\left[\frac{2}{3-\nu} \int_{0}^{1} d y \frac{\frac{2-\nu}{2}}{(1-y)^{1 / 2}}{ }_{2} F_{1}\left(\frac{1}{2}, \frac{3-v}{2} ; \frac{5-v}{2} ; y\right)\right. \\
& \left.\therefore \frac{1}{3-v} \int_{0}^{\operatorname{sen}^{2}(\alpha)} d y \frac{\frac{2-v}{2}}{(1-y)^{1 / 2}} F_{1}\left(\frac{1}{2}, \frac{3-v}{2} ; \frac{5-v}{2} ; y\right)\right]
\end{aligned}
$$

Substituinäo a expressão

$$
{ }_{2}^{F^{\prime}}{ }_{1}(a, b ; c ; y)=\frac{\Gamma(c)}{\Gamma^{\prime}(a) \Gamma(b)} \sum_{n=0}^{\infty} \frac{\Gamma^{\prime}(a+n) \Gamma(b+n)}{\Gamma^{\prime}(c+n)} \frac{y^{n}}{n !},
$$

em (4.39) e integrando em y, obtemos

$$
\begin{aligned}
& I^{\prime \prime}=-\frac{\Gamma\left(\frac{\nu-2}{2}\right) 2^{2-\nu} R^{4-\nu} 4}{8 \pi^{\nu / 2}(3-\nu)} \sum_{n=0}^{\infty} x
\end{aligned}
$$

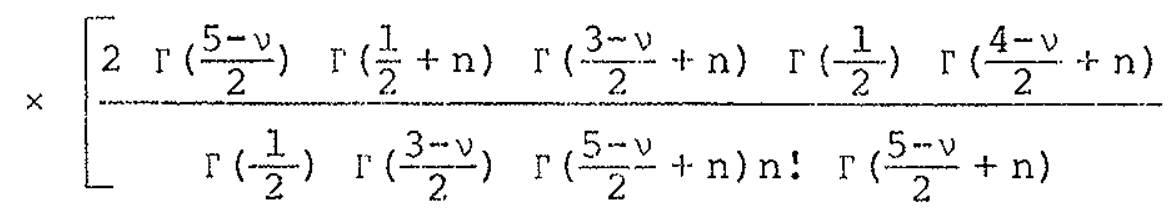

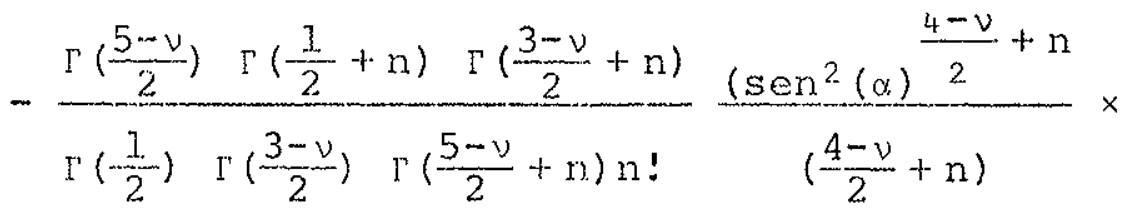

$$
\begin{aligned}
& \left.{ }_{2} F_{1}\left(\frac{1}{2}, \frac{a-v}{2}+n ; \frac{6-v}{2}+n ; \operatorname{sen}^{2}(a)\right)\right] \text {. }
\end{aligned}
$$


Observando a expressão anterior, notamos que para $n \neq 0$ e $v \leqslant 4$, não há singularidades. Então o que nos interessa è o termo da série com $n=0$, ou seja

$I^{\prime \prime \prime}=-\frac{\Gamma\left(\frac{\nu-2}{2}\right) 2^{4-\nu} R^{4-\nu}}{8 \pi \nu / 2(3-\nu)}\left[\frac{2 \Gamma\left(\frac{1}{2}\right) \Gamma\left(\frac{4-\nu}{2}\right)}{\Gamma\left(\frac{5-\nu}{2}\right)}-\frac{\left(\operatorname{sen}^{2}(\alpha)\right)^{\frac{4-\nu}{2}}}{\frac{4-\nu}{2}}\right]$.

Quando $v \rightarrow 4$ o resíduo independe de $\alpha$, como pode ser visto em (4.42), portanto podemos escolher $\alpha=\pi / 2$ e obter

$$
I^{\prime \prime \prime}=\frac{\Gamma\left(\frac{v-4}{2}\right) 2}{8 \pi^{2}(3-v)} .
$$

Finalmente, temos para a parte divergente do diagrama $G_{49}^{\prime}$

$$
\begin{aligned}
G_{4 g}^{\prime} & =\frac{n C_{2} g^{4}}{16 \pi^{2}} \Gamma\left(\frac{\nu-4}{2}\right)\left\{A-\frac{2}{3-\nu}\right\} \oint \oint d x_{1}^{\mu_{1}} d x_{2}^{\mu} 2 \times \\
& \times D_{\mu_{1} \mu_{2}}\left(x_{1}-x_{2}\right)\left|x_{1}-x_{2}\right|^{4-\nu},
\end{aligned}
$$

onde usamos o fato de que para $\alpha=\pi / 2$ o raio da circunfe rência é dado por $R=\frac{1}{2}\left|x_{1}-x_{2}\right|$.

\subsection{6 - CALCULO DO DIAGRAMA COM UM VERTICE DE TRES GLUONS}

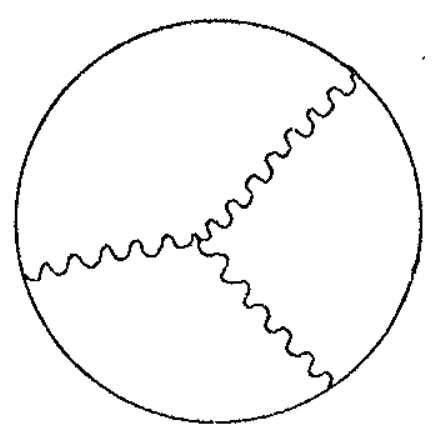


A expressão paxa esse đj.agrama é a (3.40), que está repeti da abajxo.

$$
\begin{aligned}
& G_{4 a}=-i p \frac{9^{3}}{3 !} \operatorname{Tr}\left(\lambda^{a} \lambda^{b} \lambda^{c}\right) \oint \oint \oint d x_{1}^{\mu} d x_{2}^{\nu} d x_{3}^{p} \times \\
& \times \int d^{\nu} p d^{\nu} q d^{\nu} r e^{-i\left(p x_{1}+q x_{2}+r x_{3}\right)} v_{\mu^{\prime} \nu^{\prime} p^{\prime}}^{\prime}(p, q, r) \times \\
& \times D_{\mu \mu^{\prime}}^{a a^{\prime}}(p) D_{\nu \nu^{\prime}}^{b b^{\prime}(q)} D_{\rho p^{\prime}}^{C C^{\prime}}(r) .
\end{aligned}
$$

Usando as relações $(3.20) \in(3.28)$, que são as expressões para o vërtice, propagadores e o traço das constantes de estrutura, obtemos

$$
\begin{aligned}
& G_{4 a}=-\frac{g^{4}}{3 !} P t^{a b c} \operatorname{Tr}\left(\lambda^{a} \lambda^{b} \lambda^{c}\right) \oint \oint \oint d x_{1}^{u} d x_{2}^{\nu} d x_{3}^{\rho} \times \\
& \times \int d^{\nu} p d^{\nu} q d^{\nu} r e^{-i\left(p x_{1}+q x_{2}+r x_{3}\right)} \frac{1}{(2 \pi)^{\nu}}\left\{\frac{\delta \mu^{\prime}}{p^{2}}-\right. \\
& \left.-\frac{A p_{\mu} p_{\mu}^{\prime}}{p^{4}}\right\} \frac{1}{(2 \pi)^{\nu}}\left\{\frac{\delta \nu^{\prime}}{q^{2}}-\frac{A q_{\nu} q_{\nu^{\prime}}}{q^{4}}\right\} \frac{1}{(2 \pi)}\left\{\frac{\delta p^{\prime}}{x^{2}}-\right. \\
& \left.-\frac{A r_{\rho^{\prime}} r_{\rho^{\prime}}}{r^{4}}\right\}\left\{(p-x)_{\nu^{\prime}} \delta_{\mu^{\prime} \rho^{\prime}}+(q-p)_{\rho^{\prime}} \delta_{\mu^{\prime} \nu^{\prime}+}+\right. \\
& \left.+(r-q)_{\mu}, \delta_{\nu^{\prime} \rho^{\prime}}\right\} \delta(p+q+r)
\end{aligned}
$$


o căllcujo da expressão anterior ê muito trabalhoso e exten so, devido a isso nós o efetuamos com o auxílio do progra ma SCHOONSCHIP, que foi escrito por Veltman com a finalida de calculax diagramas de Feynman. Este programa está im plantado no CDC do IEAv/CTA.

Por comodijade de programação, fizemos as se guintes alteraçôes na expressão (4.29): trocamos os momenta pelos seus simétricos e os indices $\mu, \nu, \rho$, respectivamente por $H^{\prime}, \nu^{\prime}, \rho^{\prime}$ e vice-versa, ficando

$$
\begin{aligned}
& a_{a}=\frac{9}{3 !} \mathrm{p} t^{\mathrm{abc}} \operatorname{Tr}\left(\lambda^{\mathrm{a}} \lambda^{\mathrm{b}} \lambda^{c}\right) \oint \oint \oint d x_{1}^{\mu^{\prime}} d \mathrm{x}_{2}^{\nu^{\prime}} \mathrm{d} \mathrm{x}_{3}^{\rho^{\prime}} \mathrm{x} \\
& \times \int d^{\nu} p d^{\nu} q d^{\nu} r e^{i\left(p x_{1}+q x_{2}+r x_{3}\right)}\left\{\frac{\delta \mu^{\prime}}{p^{2}}-\frac{A p_{\mu^{2}} \mu^{\prime}}{p^{4}}\right\} \times \\
& \times\left\{\frac{\delta \mu \mu^{\prime}}{q^{2}}-\frac{A q_{\nu} q_{\nu^{\prime}}}{q^{4}}\right\}\left\{\frac{\delta \rho^{\prime}}{r^{2}}-\frac{A r_{\rho^{r}} \rho^{\prime}}{r^{4}}\right\}\left\{(p-q)_{\rho} \delta_{\mu \nu}+\right. \\
& \left.+(q-r)_{\mu} \delta_{\rho \nu}+(x-p)_{\nu} \delta_{\rho \mu}\right\} \text {. }
\end{aligned}
$$

Como ê de conhecimento geral não é comum, pelo menos atual mente, existir simbolos gregos em computador, portanto fi zemos as mudanças $\mu^{\prime}, \nu^{\prime}, \rho^{\prime}, \mu, \nu, \rho$ por respectivamente UL, VL, RL, U, V, RO; $\circ \vee$ da dimensão foi substituído por T. No programa, a componente $\mu$ do vetor p é escrita na forma $P(U)$, o produto escalar entre $p$ e $q$ é PDQ e a delta de Kronecker tem a forma $D(U, V)=\delta_{\mu \nu}$.

o programa que calcula (4.30) está na integra no apêndice $B$ e contém comentários elucidativos. O símbolo CIM do programa vale 


$$
\operatorname{CrM}=\frac{q^{l_{4}}}{3 !} \delta(p+q+r) p t^{a b c} \operatorname{Ir}^{a}\left(\lambda^{a} \lambda^{b} \lambda^{c}\right)
$$

A constante de estrutura $t^{a b c}$ e gerador $\lambda^{a}$ são represen tados por $\mathrm{T}(A, B, C)$ e IA, respectivamente. Com esse progra ma obtivemos

$$
\begin{aligned}
\mathrm{G}_{4 \mathrm{a}}= & \frac{\mathrm{g}^{4} \mathrm{nC} \mathrm{C}_{2} \Gamma(\nu-3) \Gamma\left(2-\frac{v}{2}\right) \Gamma\left(\frac{v}{2}-1\right) \Gamma\left(\frac{\nu}{2}-1\right) \mathrm{A}}{2^{6}{ }_{\pi}^{\nu} \Gamma\left(\frac{6-\nu}{2}\right) \Gamma(\nu-2)} \times \\
& \times\left\{2-\frac{13}{2}-\frac{1}{4} \mathrm{~A} \nu+\mathrm{A}\right\} \times \\
& \times \oint \oint \mathrm{dx} \mathrm{dx}_{2}^{\mu} \delta_{\mu \nu}\left|\mathrm{x}_{2}-\mathrm{x}_{1}\right|^{6-2 \nu} .
\end{aligned}
$$

\subsection{7 - CALCULO DO DIAGIRAMA DE DOTS VERTICES COM TRES GLIJONS}

A expressão para esse diagrama é

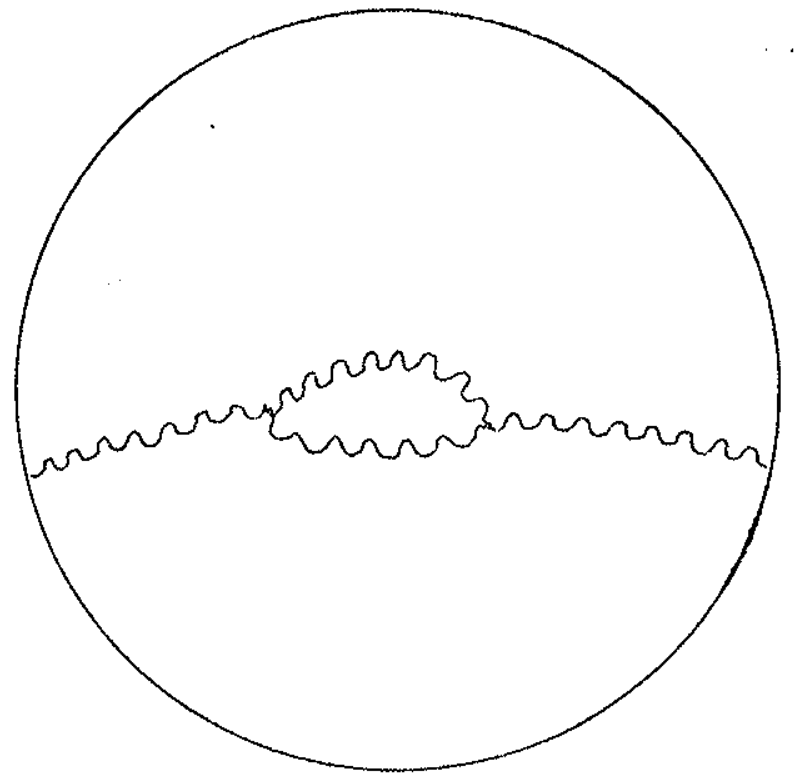


ou

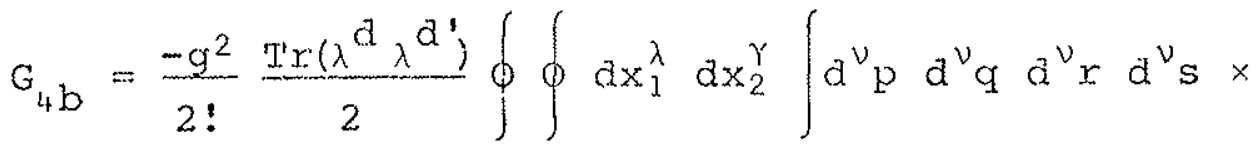

$$
\begin{aligned}
& \times e^{i\left(s x_{1}-r x_{2}\right)} v_{\mu_{1} \rho v_{1}}^{a_{1} c_{1} b_{1}}(q,-s,-p) v_{\mu_{2} v_{2} \rho_{2}}^{a_{2} b_{2} c_{2}}(-q, p, r) \times \\
& \times D_{\lambda p_{1}}^{d c_{1}}(s) \quad D_{v_{1} v_{2}}^{b_{1} b_{2}(p)} D_{\mu_{1} \mu_{2}}^{a_{1} a_{2}}(q) D_{\rho_{2} \gamma}^{C_{2} d^{\prime}}(r)
\end{aligned}
$$

Substituindo as expressões $(3.20),(3.28)$ e $(4.21)$ na ex pressão anterior temos

$$
\begin{aligned}
& G_{4 b}=-\frac{g^{4} n C_{2}}{2} \oint \oint d x_{1}^{\lambda} d x_{2}^{\gamma} \int d^{\nu} p d^{\nu} q d^{\nu} r a^{\nu} s e^{i\left(s x_{1}-r x_{2}\right)} x \\
& \times(2 \pi)^{2 \nu} \delta(q \cdots s-p) \delta(p+x-q)\left\{(q+p)_{\rho_{1}} \delta_{\mu_{1} \nu_{1}}+\right. \\
& \left.+(-s-q)_{\nu_{1}} \delta_{\rho_{1} \mu_{1}}+(-p+s)_{\mu_{1}} \delta_{\rho_{1} \nu_{1}}\right\}\left\{(-q-r)_{\nu_{2}} \delta_{\mu_{2} \rho_{2}}+\right. \\
& \left.+(p+q)_{\rho_{2}} \delta_{\mu_{2} \nu_{2}}+(x-p)_{\mu_{2}} \delta_{\nu_{2} \rho_{2}}\right\} \frac{1}{(2 \pi)^{4 \nu}} \times \\
& \times\left\{\frac{\delta \rho_{\rho_{1}}}{s^{2}}-\frac{A s_{\lambda} s_{\rho}}{s^{4}}\right\}\left\{\frac{\delta_{\nu_{1} \nu_{2}}}{p^{2}}-\frac{A p_{\nu_{1}} P_{\nu_{2}}}{p^{4}}\right\} \\
& \times\left\{\frac{\delta_{\mu_{1} \mu_{2}}}{q^{2}}-\frac{A q_{\mu_{1}} q_{\mu_{2}}}{q^{4}}\right\}\left\{\frac{\delta_{\rho_{2} \gamma}}{r^{2}}-\frac{A r_{2} r_{\gamma}}{r^{4}}\right\}
\end{aligned}
$$


E conveniente, neste ponto, continuar o cälculo com a aju da do SCHOONSCHIP, como foi feito na seção anterior. o cál. culo se encontra no apendice $C$. No programa fizemos as se guintes trocas: $\lambda_{1}, \mu_{1}, \mu_{2}, \nu_{1}, \nu_{2}, \rho_{1}, \rho_{2}, \gamma, \nu$, respecti vamente por, I, U1, U2, V1, V2, R1, R2, GA, T. Representa mos por CI'M todas as constantes globais e algumas varia veis não usadas em uma determinada etapa. Inicialmente te mos

$$
\operatorname{CTM}=-\frac{q^{4} n C_{2}}{2(2 \pi)^{2 T}} \delta(q-s-p) \delta(p+r-q)
$$

Feito os cálculos temos

$$
\begin{aligned}
G_{4 b} & =-\frac{g^{4} n C_{2} \Gamma(\nu-3) \Gamma\left(2-\frac{v}{2}\right) \Gamma\left(\frac{v}{2}-1\right) \Gamma\left(\frac{v}{2}-1\right)}{128 \pi^{\nu} \Gamma\left(3-\frac{v}{2}\right) \Gamma(\nu-2)} \times \\
& \times \frac{6 v-5}{2(\nu-1)}+A(2 \nu-7)-A\left(1-\frac{v}{4}\right) \times \\
& \times \oint \oint d x_{1}^{\mu} d x_{2}^{v}\left|x_{2}-x_{1}\right|^{6-2 \nu} \delta_{\mu \nu} .
\end{aligned}
$$

\section{2 .8 - RENORMALIZAÇÃO DA CARGA}

Vamos reescrever os resultados dos diagramas não abelianos calculados nas seções anteriores.

$G_{49}=$

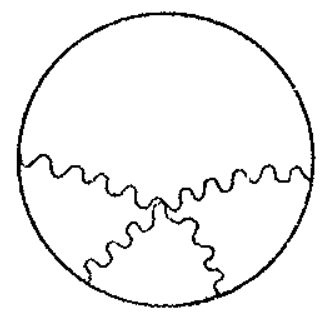

$$
\begin{aligned}
& =\frac{\mathrm{nC}_{2} \mathrm{~g}^{4}}{16 \pi / 2} \Gamma\left(\frac{v-4}{2}\right)\left\{A-\frac{2}{3-v}\right\} \times \\
& \times \oint \phi d x^{\sigma} d y^{\rho} D_{\sigma \rho}(x-y)|x-y|^{4-\nu},
\end{aligned}
$$



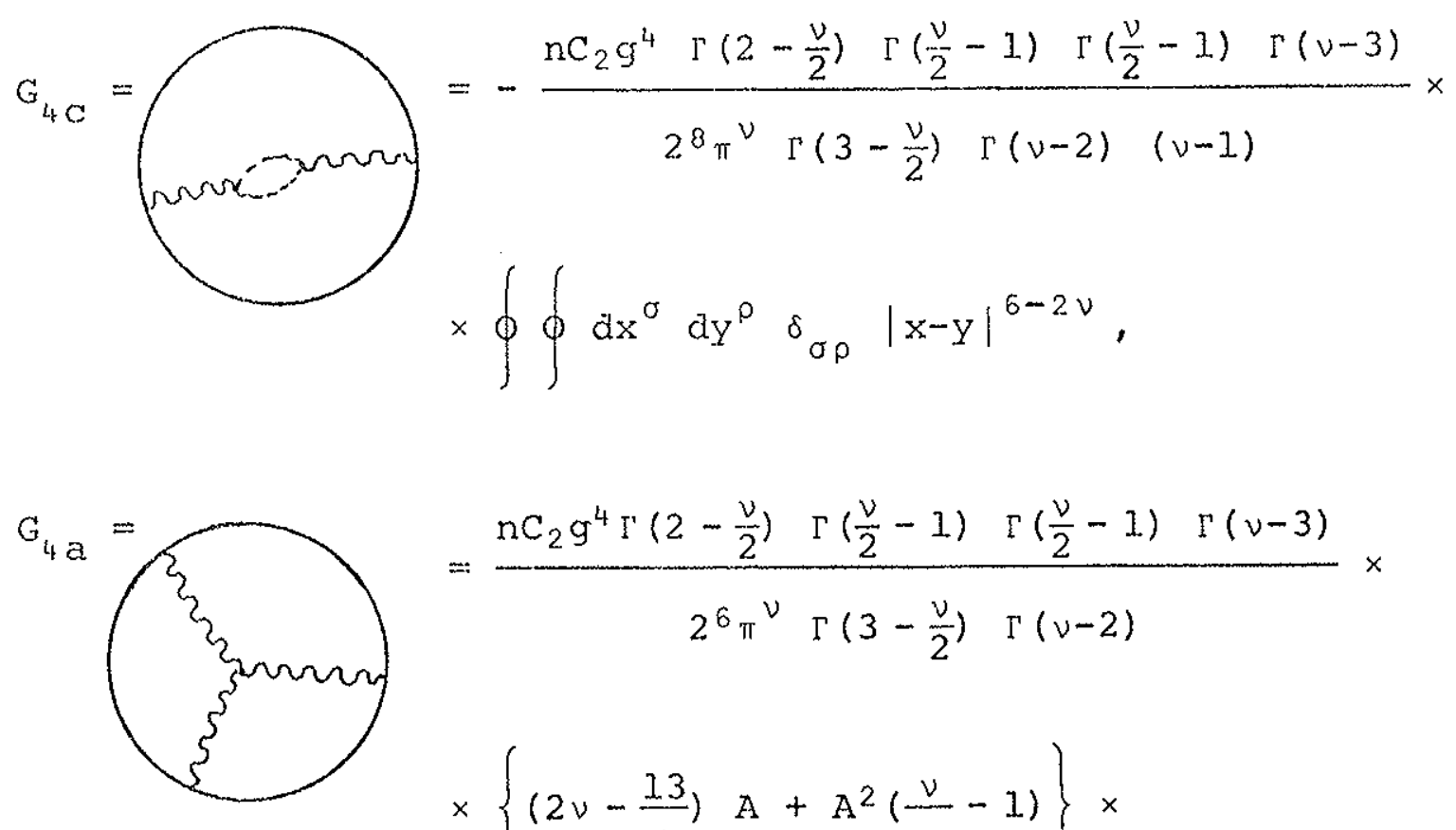

$$
\begin{aligned}
& =\frac{n C_{2} g^{4} \Gamma^{1}\left(2-\frac{\nu}{2}\right) \Gamma\left(\frac{\nu}{2}-1\right) \Gamma\left(\frac{\nu}{2}-1\right) \Gamma(\nu-3)}{2^{6} \pi^{\nu} \Gamma\left(3-\frac{v}{2}\right) \Gamma(\nu-2)} \times \\
& \times\left\{\left(2 \nu-\frac{13}{2}\right) A+A^{2}\left(\frac{\nu}{4}-1\right)\right\} \times
\end{aligned}
$$$$
x \oint \oint d x^{\sigma} d y^{\rho} \delta_{\sigma \rho}|x-y|^{6-2 \nu},
$$

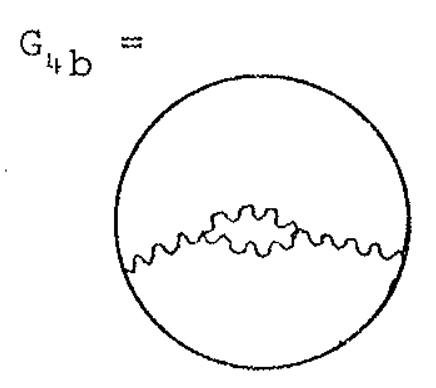

$$
\begin{aligned}
& =\frac{\mathrm{nC}_{2} \mathrm{~g}^{4} \Gamma^{\prime}\left(2-\frac{\nu}{2}\right) \Gamma\left(\frac{\nu}{2}-1\right) \Gamma\left(\frac{\nu}{2}-1\right) \Gamma(\nu-3)}{2^{7} \pi^{\nu} \Gamma\left(3-\frac{v}{2}\right) \Gamma(\nu-2)} \times \\
& \times\left\{\frac{6 v-5}{2(\nu-1)}+(2 \nu-7) \mathrm{A}-\left(1-\frac{\nu}{4}\right) \mathrm{A}^{2}\right\} \times \\
& \times \oint_{\mathrm{d} \mathrm{x}^{\sigma} \mathrm{d} \mathrm{x}^{\rho} \delta_{\sigma \rho}|\mathrm{x}-\mathrm{y}|^{6-2 \nu} .}
\end{aligned}
$$

onde $\mathrm{x}$ e $\mathrm{y}$ estão no lugar de $\mathrm{x}_{1} \in \mathrm{x}_{2}$ e $\rho$ e $\sigma$ são Indices de Lorentz. Para dimensões diferentes de quatro não pode mos exprimir os resultados dos diagramas $G_{4 a}, G_{4_{4} b}, G_{4} \mathrm{C}$ em termos do propagador, desta forma concluimos que me rece maior atenção tratar a renormalização em di 
mensões espaço-temporais diferentes de 4 . Fazendo o limi. te $v \rightarrow 4$ em (4.55), abtemos

$$
\begin{aligned}
& G_{4 a}+G_{4 b}+G_{4 c}+G_{4 g}=\frac{n C_{2} g^{4}}{\pi^{2}(4-v)}\left\{-\frac{19}{2^{5} \times 3}-\frac{A}{2^{4}}+\frac{3 A}{2^{4}}-\frac{1}{3 \times 2^{5}}-\right. \\
& \left.-\frac{A}{8}-\frac{J}{4}\right\} \oint \oint d x^{\sigma} d y^{\rho} D_{\sigma \rho}(x-y)=-\frac{I 1}{24} \frac{n C_{2} g^{4}}{\pi^{2}(4-\nu)} \times \\
& \quad \times \oint \phi d x^{\sigma} d y^{\rho} D_{\sigma \rho}(x-y) .
\end{aligned}
$$

Somando esse resultado com o de segunda ordem (4.3), temos

$$
\begin{aligned}
& G_{2}+G_{4 a}+G_{4 b}+G_{4 C}+G_{4 g}=-n g^{2}\left(1+\frac{11 C_{2} g^{2}}{24 \pi^{2}(4-v)}\right) \times \\
& \quad \times \oint \oint d x^{\sigma} d y^{\rho} D_{\sigma \rho}(x-y)
\end{aligned}
$$

Até o momento omitimos nas expressões uma constante dimen sional. $\mu$. Isto é necessário pois devemos fazer cálculos perturbativos em parâmetros adimensionais. Portanto, nes ses cálculos $g$ deve ser entendido como sendo gu $\frac{v-4}{2}$. Na ex pressäo a seguir $g_{0}$ é a carga nua da teoria e g é a carga renormalizada. Finalmente temos

$$
g^{2}=\mu^{\nu-4} g_{0}^{2}\left\{1+\frac{J 1 C_{2} g_{0}^{2} \mu^{\nu-4}}{24 \pi^{2}(4-\nu)}\right\},
$$

que è a expressão que dá a carga renormalizada da cromodi nâmica quântica em quarta ordem. 


\section{CAPTTUTO 5}

PROVA DA INDEPENDENCIA DO RESTDUO EM RELACCÃO AO ARCO E A CURVA E CALCULO DA HIPERBOLE EM SEGUNDA ORDEM

Neste capitulo mostramos que em quatro dimen sões o resĩuo que se obtem da integração do fator de fase quántico sobre uma curva aberta e lisa, não depende do ax. co nem da curva.

Em seguida calculamos este fator de fase pa ra a hipérbole no gauge de Feynman.

\section{1 - INDEPENDENCIA DO RESIDUO EM RELAC̆ÃO A CURVA E AO ARCO}

A divergência resultante da integração do fa tor de fase quântico, sobre a curva, se origina quando os pontos $x$ e $y$ estão próximos. Este Eato pode ser observado no primeiro termo do propagador (eq. 5.1), que no limite $\nu \rightarrow 4$, tem a forma $1 /|x-y|^{2}$. Por esse motivo sö precisamos nos preocupar com pequenos arcos da curva. Neste caso pode mos tratä-m los como se fossem arcos de circunferência. A se gujr calculamos em segunäa ordem a integração mencionada anteriormente. O propagador no espaço de coordenadas tem a forma,

$$
D_{\mu \nu}(x-y)=\frac{\Gamma\left(\frac{\nu-2}{2}\right)}{4 \pi^{\nu / 2}}\left\{\delta_{\mu \nu}|x-y|^{2-\nu}-\frac{A \partial_{\mu}^{x} \partial}{2(\nu-4)}|x-y|^{4-\nu}\right\} .
$$

Para obter esse propagador no gauge de feynman fazemos $\mathrm{A}=0$. o nosso objetivo, a seguir, è calcular a integral 


$$
V(C)=\frac{\Gamma\left(\frac{\nu-2}{2}\right)}{4 \pi^{\nu / 2}} \int_{C} d x^{\mu} d y^{\nu} \delta_{\mu \nu}|x-y|^{2-\nu}
$$

onde $\mathrm{C}$ è o arco de circunferência. Para esclarecer melhor, mostramos na fig. 5.1 o arco C simétrico com relação ao es xo (1)

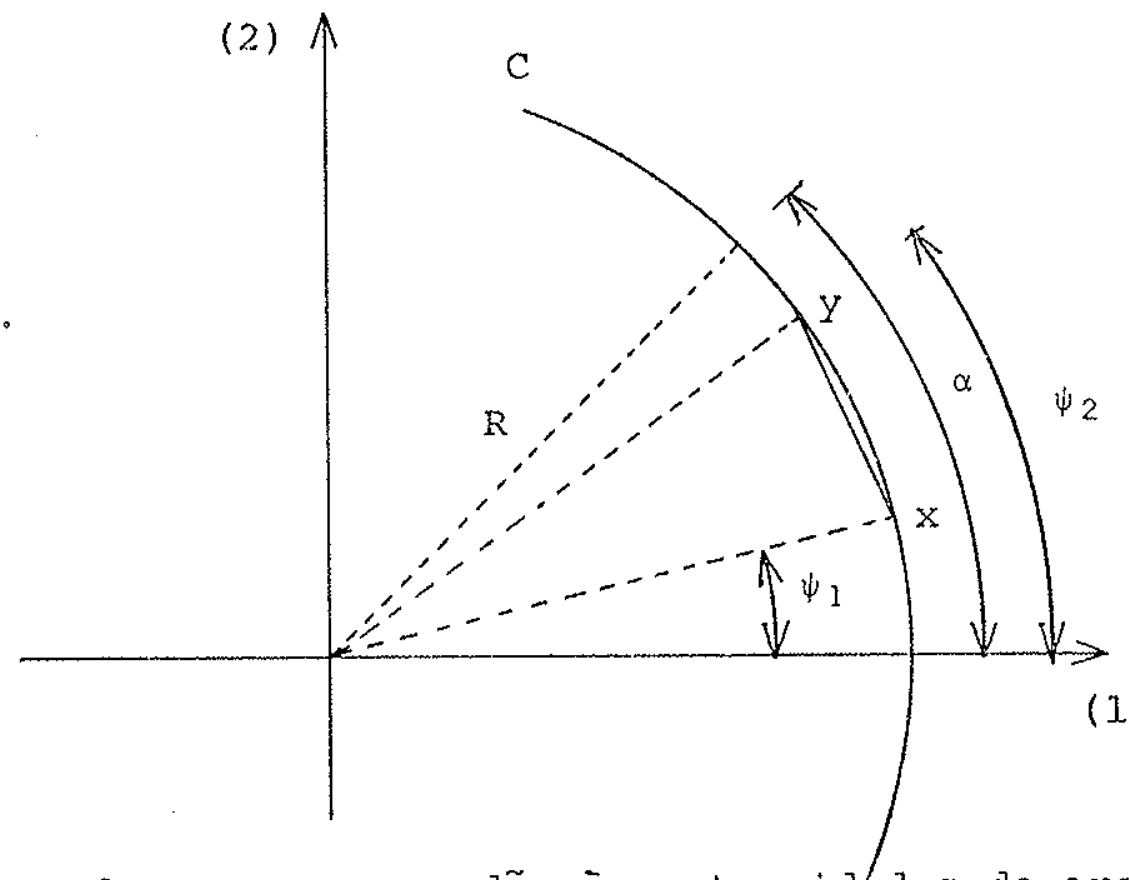

fig. 5.1

Os angulos $\alpha$ e $-\alpha$ nos dão às extremidades do arco C. Os pon tos $x$ e $y$ ficam completamente determjnados por $R$ (raio do axco) e respectivamente pelos ângulos $\psi_{1}$ e $\psi_{2}$, que são as novas variáveis. Então as componentes de $x$ e $y$ nas dire ções (1) e (2) que são $x_{1}, x_{2}, y_{1}, y_{2}$ são dadas por

$$
\begin{aligned}
& x_{1}=\operatorname{RCos}\left(\psi_{1}\right), \\
& x_{2}=\operatorname{Rsen}\left(\psi_{1}\right), \\
& y_{1}=\operatorname{Rcos}\left(\psi_{2}\right), \\
& y_{2}=\operatorname{Rsen}\left(\psi_{2}\right),
\end{aligned}
$$

e suas diferenciais são 


$$
\begin{aligned}
& d x_{1}=-\operatorname{Rsen}\left(\psi_{1}\right) d \psi_{1}, \\
& d x_{2}=\operatorname{Rcos}\left(\psi_{1}\right) d \psi_{1}, \\
& d y_{1}=-\operatorname{Rsen}\left(\psi_{2}\right) d \psi_{2}, \\
& d y_{2}=\operatorname{Rcos}\left(\psi_{2}\right) d \psi_{2},
\end{aligned}
$$

substituindo as equações (5.3) e (5.4) na equação (5.2) e sabendo que $|x-y|^{2}=4 R^{2} \operatorname{sen}^{2}\left(\left(\psi_{1}-\psi_{2}\right) / 2\right)$, temos

$$
\begin{aligned}
V(C) & =\frac{\Gamma\left(\frac{\nu-2}{2}\right)}{4 \pi^{\nu / 2}} 2^{2-\nu} R^{4-\nu} \int_{-\alpha}^{\alpha} d \psi_{1} \int_{-\alpha}^{\alpha} d \psi_{2} x \\
& \times\left(\operatorname{sen}^{2}\left(\frac{\psi_{1}-\psi_{2}}{2}\right)^{\frac{2-\nu}{2}} \cos \left(\psi_{1}-\psi_{2}\right) .\right.
\end{aligned}
$$

Substituindo

$$
a=\frac{\Gamma\left(\frac{\nu-2}{2}\right) 2^{2-v} R^{4-v}}{4 \pi^{\nu / 2}},
$$

e

$$
\cos \left(\psi_{1}-\psi_{2}\right)=1-2 \operatorname{sen}^{2}\left(\frac{\psi_{1}-\psi_{2}}{2}\right)
$$

em (5.5) seque que

$$
\begin{aligned}
V(C) & =a \int_{-\alpha}^{\alpha} d \psi_{1} \int_{-\alpha}^{\alpha} d \psi_{2}\left(\operatorname{sen}^{2}\left(\frac{\psi_{1}-\psi_{2}}{2}\right)\right)^{\frac{2-v}{2}}- \\
& -2 a \int_{-\alpha}^{\alpha} d \psi_{1} \int_{-\alpha}^{\alpha} d \psi_{2}\left(\operatorname{sen}^{2}\left(\frac{\psi_{1}-\psi_{2}}{2}\right)\right)^{\frac{4-\nu}{2}} .
\end{aligned}
$$


Fazendo as substitujções

$$
\begin{aligned}
& \operatorname{sen}\left(\frac{\psi_{1}-\psi_{2}}{2}\right) \frac{2 \tan \left(\frac{\psi_{1}-\psi_{2}}{2}\right)}{1+\tan ^{2}\left(\frac{\psi_{1}-\psi_{2}}{4}\right)}, \\
& t=\tan \left(\frac{\psi_{1}-\psi_{2}}{2}\right) \\
& d t=\left(1+t^{2}\right) \frac{d \psi_{1}}{4},
\end{aligned}
$$

resulta em

$$
\begin{aligned}
V(C)=a & \int_{-\alpha}^{\alpha} d \psi_{2}\left\{\int_{-\tan \left(\frac{\alpha+\psi_{2}}{4}\right)}^{\tan \left(\frac{\alpha-\psi_{2}}{4}\right)} d t \frac{4}{1+t^{2}}\left[\left(\frac{2 t}{1+t^{2}}\right)^{2}\right]^{\frac{2-\nu}{2}}-\right. \\
& \left.-2 \int_{-\tan \left(\frac{\alpha+\psi_{2}}{4}\right)}^{\tan \left(\frac{\alpha-\psi_{2}}{4}\right)} \frac{4}{1+t^{2}}\left[\left(\frac{2 t}{1+t^{2}}\right)^{2}\right]^{\frac{4-\nu}{2}}\right\} .
\end{aligned}
$$

Fazendo a mudança de variavel $y=t^{2}$ na expressão anterior e reescrevendo as integrais em uma forma mais conveniente, obtemos

$$
V(C)=\frac{2^{4-\nu} a}{2} \int_{-\alpha}^{\alpha} d \psi_{2}\left\{\int_{0}^{\tan ^{2}\left(\frac{\alpha-\psi_{2}}{4}\right)} d y \frac{y^{2}}{(1+y)^{3-\nu}}+\right.
$$




$$
\begin{aligned}
& +\int_{0}^{\tan ^{2}\left(\frac{\alpha-\psi_{2}}{4}\right)} d y \frac{\frac{1-v}{2}}{(1+y)^{3-v}-} \\
& \left.-8 \int_{0}^{\tan ^{2}\left(\frac{\alpha-\psi_{2}}{4}\right)} d y \frac{\frac{y^{\frac{3-v}{2}}}{(1+y)^{5-v}}-}{-8} \int_{0}^{\tan ^{2}\left(\frac{\alpha-\psi_{2}}{4}\right)} d y \frac{y^{2}}{(1+y)^{3-v}}\right\} .
\end{aligned}
$$

A expressão anterior è par em $\psi_{2}$. Usando este fato e inte grando em y temos

$$
\begin{aligned}
& V(C)=2^{4-v} a \int_{0}^{\alpha} d \psi_{2}\left\{\frac{\left(\tan ^{2}\left(\frac{\alpha-\psi_{2}}{4}\right)\right)^{\frac{3-\nu}{2}}}{\frac{3-v}{2}}{ }^{\frac{3-\nu}{v}} F_{1}\left(3-v, \frac{3-v}{2} ; \frac{5-v}{2} ;-\tan ^{2}\left(\frac{\alpha-\psi_{2}}{4}\right)\right)+\right. \\
& +\frac{\left(\tan ^{2}\left(\frac{\alpha+\psi_{2}}{4}\right)\right)^{\frac{3-\nu}{2}}}{\frac{3-v}{2}} 2^{F_{1}}\left(3-v, \frac{3-v}{2} ; \frac{5-v}{2} ;-\tan ^{2}\left(\frac{\alpha+\psi 2}{4}\right)\right)- \\
& -8 \frac{\left(\tan ^{2}\left(\frac{\alpha-\psi_{2}}{4}\right)\right)^{\frac{5-\nu}{2}}}{\frac{5-v}{2}}{ }_{2} F_{1}\left(5-v, \frac{5-v}{2} ; \frac{7-v}{2} ;-\tan ^{2}\left(\frac{\alpha-\psi_{2}}{4}\right)\right)- \\
& \left.-8 \frac{\left(\tan ^{2}\left(\frac{\alpha+\psi_{2}}{4}\right)\right)^{\frac{5-v}{2}}}{\frac{5-v}{2}} 2 F_{1}\left(5-v, \frac{5-v}{2} ; \frac{7-v}{2} ;-\tan ^{2}\left(\frac{\alpha+\psi_{2}}{4}\right)\right)\right\} .
\end{aligned}
$$


Fazendo as mudanças de variáveis

$x=\tan \left(\frac{\alpha-\psi_{2}}{4}\right), \quad d x=-\left(1+\tan ^{2}\left(\frac{\alpha-\psi_{2}}{4}\right)\right) \frac{d \psi_{2}}{4}$,

no primeiro e no terceiro termos da expressão (5.14) e

$x=\tan \left(\frac{\alpha+\psi_{2}}{4}\right), \quad d x=\left(1+\tan ^{2}\left(\frac{\alpha+\psi_{2}}{4}\right)\right) \frac{d \psi_{2}}{4}$,

no segundo e no quarto termos, temos

$$
\begin{aligned}
v(C)=22^{7-v} a & \left\{\int_{0}^{\tan \left(\frac{\alpha}{2}\right)} d x \frac{x^{3-v}}{(3-v)\left(1+x^{2}\right)}{ }^{2} F_{1}\left(3-v, \frac{3-v}{2} ; \frac{5-v}{2} ;-x^{2}\right)-\right. \\
& \left.-8 \int_{0}^{\tan \left(\frac{\alpha}{2}\right)} d x \frac{x^{5-v}}{(5-v)\left(1+x^{2}\right)} 2^{F_{1}}\left(5-v, \frac{5-v}{2} ; \frac{7-v}{2} ;-x^{2}\right)\right\} .
\end{aligned}
$$

Como $\alpha$ é um ângulo pequeno, temos $x<1$, e portanto podemos substituir a hipergeométrica pela sua expansão em série:

$$
{ }_{2} F_{1}(a, b ; c ; z)=\sum_{n=0}^{\infty} \frac{(a)_{n}(b)_{n}}{(c)_{n}} \frac{z^{n}}{n !} .
$$

Resultando em

$$
V(C)=2^{7-v} a\left\{\int _ { 0 } ^ { \operatorname { t a n } ( \frac { \alpha } { 2 } ) } d x \frac { ( x ^ { 2 } ) ^ { \frac { 3 - \nu } { 2 } } } { ( 3 - ) ( 1 + x ^ { 2 } ) } \left[1+\frac{(3-v)\left(\frac{3-v}{2}\right)}{\frac{5-v}{2}}\left(-x^{2}\right)+\right.\right.
$$




$$
\begin{aligned}
& \left.+\frac{(3-v)(4-v)\left(\frac{3-v}{2}\right)\left(\frac{5-v}{2}\right)\left(-x^{2}\right)^{2}}{2 !\left(\frac{5-v}{2}\right)\left(\frac{7-v}{2}\right)}\right]- \\
& -8 \int_{0}^{\tan \left(\frac{x}{2}\right)} d x \frac{\left(x^{2}\right)^{\frac{5-v}{2}}}{(5-v)\left(1+x^{2}\right)}\left[1+\frac{(5-v)\left(\frac{5-v}{2}\right)}{\left(\frac{7-v}{2}\right)}\left(-x^{2}\right)+\right. \\
& \left.\left.+\frac{(5-v)(6-v)\left(\frac{5-v}{2}\right)\left(\frac{7-v}{2}\right)\left(-x^{2}\right)^{2}}{2 !\left(\frac{7-v}{2}\right)\left(\frac{9-v}{2}\right)}+\ldots\right]\right\}
\end{aligned}
$$

Integrando em $x$ e levando em conta que a é dado por $(5.6)$ temos

$$
\begin{aligned}
& V(C)=\frac{2^{8-2 \nu} \Gamma\left(\frac{v-2}{2}\right) R^{4-v}}{8 \pi v / 2}\left\{\frac{\left(\tan ^{2}\left(\frac{\alpha}{2}\right)^{\frac{4-v}{2}}\right.}{(3-v)\left(\frac{4-v}{2}\right)} \times\right. \\
& \times 2 \mathrm{~F}_{1}\left(1, \frac{4-v}{2} ; \frac{6-v}{2} ;-\tan ^{2}\left(\frac{\alpha}{2}\right)\right)+ \\
& +\frac{-\frac{3-\nu}{2}\left(\tan ^{2}\left(\frac{\alpha}{2}\right)\right)^{\frac{6-\nu}{2}}}{\left(\frac{5-\nu}{2}\right)\left(\frac{6-v}{2}\right)}{ }_{2} F_{1}\left(1, \frac{6-\nu}{2} ; \frac{8-\nu}{2} ;-\tan ^{2}\left(\frac{\alpha}{2}\right)+\ldots\right. \\
& -\frac{8\left(\tan ^{2}\left(\frac{\alpha}{2}\right)\right)^{\frac{6-v}{2}}}{(5-v)\left(\frac{6-v}{2}\right)} 2 F_{1}\left(1, \frac{6-v}{2} ; \frac{8-v}{2} ;-\tan ^{2}\left(\frac{\alpha}{2}\right)+\ldots\right\} .
\end{aligned}
$$


Nessa expressão somente o primeiro termo é divergente quan do a dimensão $(v)$ tende para quatro. Portanto é o termo que nos interessa. Nesse limite a hipergeométrica tem o valor

$$
\lim _{v \rightarrow 4} F_{1}\left(1, \frac{4-v}{2} ; \frac{6-v}{2} ;-\tan 2 \cdot\left(\frac{\alpha}{2}\right)\right)=1
$$

o resíduo de $V(C)$ no pol.o $v=4$ é dado por

$\operatorname{Res} V(C)=\lim _{v \rightarrow 4} \frac{(v-4) 2^{8-2 v} \cdot I^{\prime}\left(\frac{v-2}{2}\right) R^{4-v}\left(\tan ^{2}\left(\frac{\alpha}{2}\right)\right)^{\frac{4-v}{2}}}{4 \pi^{v / 2}(3-v) \frac{4-v}{2}}=\frac{1}{2 \pi^{2}}$,

Portanto $V(C)$ näo depende de $\alpha$ para $\nu=4$. Ficando deste mo do mostrado o que queriamos.

\section{2 - CALCUISO DE V(C) PARA A HIPERBOLE}

Nesta seçio calculamos a parte finita e o re siduo para a hipérbole da figura abaixo.

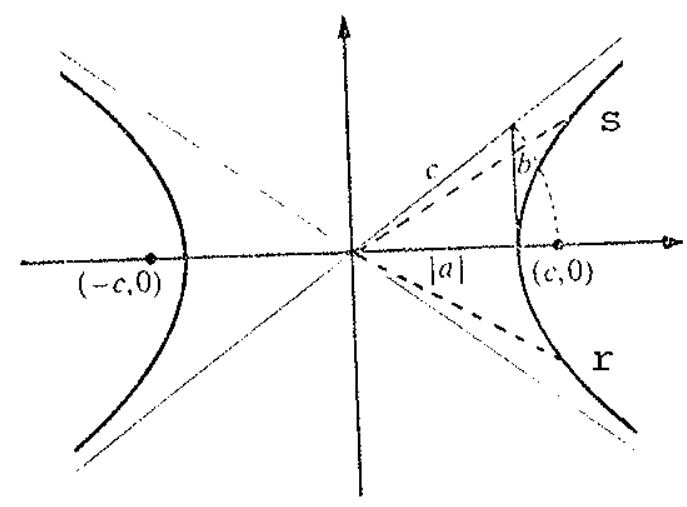

Eig. 5.2 
onde $r$ es são pontos da hipérbole cuja equação é dada por

$$
\frac{x_{x}^{2}}{a^{2}}-\frac{x_{y}^{2}}{b^{2}}=1 \text { ou } \frac{s_{x}^{2}}{a^{2}}-\frac{s_{y}^{2}}{b^{2}}=1,
$$

sendo $r_{x}$ e $r_{y}, s_{x}$ e $s_{y}$ são as componentes de $r$ e $s$ nas dire ções $x$ e y. A equação da hipérbole pode ser parametrizada da seguinte maneira:

$$
\begin{aligned}
& x_{\mathrm{x}}=\operatorname{acosh}(\alpha) \quad \mathrm{s}_{\mathrm{x}}=\operatorname{acosh}(\beta) \\
& r_{y}=b \operatorname{senh}(\alpha) \quad s_{y}=b \operatorname{senh}(\beta) \\
& \text { O nosso intuito é calcular } V(C) \text { ou seja } \\
& V(C)=\iint d x^{\mu} d s^{\nu} D_{\mu \nu}(s-x) .
\end{aligned}
$$

A expressão para o propagador é dada pela eq. (5.1). No gau ge de Feynman, temos

$$
V(C)=\frac{\Gamma\left(\frac{v-2}{2}\right)}{4 \pi^{\nu / 2}} \iint d r^{\mu} d s^{\nu} \delta_{\mu \nu}|r-s|^{2-\nu} .
$$

Substituindo as equações $(5.23)$ em $(5.25)$, temos

$$
\begin{aligned}
V(C) & =\frac{\Gamma\left(\frac{v-2}{2}\right)}{4 \pi^{v / 2}} \int_{-\infty}^{\infty} \int_{-\infty}^{\infty} d a d \beta\left(a^{2} \operatorname{senh}(\alpha) \operatorname{senh}(\beta)+\right. \\
& \left.+b^{2} \cosh (\alpha) \cosh (\beta)\right)\left[a^{2}(\cosh (\beta)-\cosh (\alpha))^{2}+\right. \\
& \left.+b^{2}(\operatorname{senh}(\beta)-\operatorname{senh}(\alpha))^{2}\right]^{\frac{2-v}{2}} .
\end{aligned}
$$


Mantendo constante a relação b/a, temos una ramilia de hi. pérboles com as mesmas assintotás. Substituindo as relações

$$
\cosh (\beta)-\cosh (\alpha)=-2 \operatorname{senh}\left(\frac{\alpha+\beta}{2}\right) \operatorname{senh}\left(\frac{\alpha-\beta}{2}\right)
$$

e

$$
\operatorname{senh}(\beta)-\operatorname{senh}(\alpha)=-2 \operatorname{senh}\left(\frac{\alpha-\beta}{2}\right) \operatorname{senh}\left(\frac{\alpha+\beta}{2}\right)
$$

no fator entre colchetes da equação (5.26) e substituindo

$$
\cos (\alpha) \cosh (\beta)=\frac{1}{2}[\cosh (\alpha+\beta)+\cosh (\alpha-\beta)]
$$

e

$$
\operatorname{sen}(\alpha) \operatorname{senh}(\beta)=\frac{1}{2}[\cosh (\alpha+\beta)-\cosh (\alpha-\beta)]
$$

no primeiro fator temos

$$
\begin{aligned}
V(C) & =\frac{\Gamma\left(\frac{\nu-2}{2}\right)}{4 \pi^{\nu / 2}}\left(a^{2}\right)^{\frac{4-\nu}{2}} \int_{-\infty}^{\infty} \int_{-\infty}^{\infty} \frac{d \alpha d \beta}{2}(\cos (\nu+\beta)-\cosh (\alpha-\beta)+ \\
& +\tan ^{2}(x)(\cosh (\alpha+\beta)+\cosh (\alpha-\beta))\left(4 \operatorname{sen}^{2}\left(\frac{\alpha-\beta}{2}\right)\right)^{\frac{2-\nu}{2}} \times \\
& \times\left[\operatorname{senh}^{2}\left(\frac{\alpha+\beta}{2}\right)+\tan ^{2}(x) \cosh ^{2}\left(\frac{\alpha+\beta}{2}\right)\right)^{\frac{2-\nu}{2}},
\end{aligned}
$$

onde 


$$
\tan (x)=\frac{b}{a}
$$

Fazendo a mudança de variäveis

$$
u=\frac{\alpha+\beta}{2} \in v=\frac{\alpha-\beta}{2},
$$

obtemos

$$
\begin{aligned}
& V(C)=\frac{4^{\frac{2-\nu}{2}} \Gamma \cdot\left(\frac{\nu-2}{2}\right)\left(a^{2}\right)^{\frac{4-\nu}{2}}\left(\sec ^{2}(x)^{\frac{2-\nu}{2}}\right.}{4 \pi^{v / 2}}\left\{\int_{-\infty}^{\infty} \int_{-\infty}^{\infty} d u d v x\right. \\
& x \sec ^{2}(x) \cosh (2 u)\left(\operatorname{senh}^{2}(2 v)\right)^{\frac{2-v}{2}}\left(\cosh ^{2}(u)-\cosh ^{2}(x)^{\frac{2-v}{2}}+\right. \\
& +\int_{-\infty}^{\infty} \int_{-\infty}^{\infty} d u d v\left(\tan ^{2}(x)-1\right) \cosh (2 v)\left(\operatorname{senh}^{2}(v)\right)^{\frac{2-\nu}{2}} x \\
& \left.\times\left(\cosh ^{2}(u)-\cos ^{2}(x)\right)^{\frac{2-v}{2}}\right\}
\end{aligned}
$$

ou

$V(C)=\frac{4^{\frac{4-\nu}{2}} \Gamma\left(\frac{\nu-2}{2}\right)\left(a^{2}\right)^{\frac{4-\nu}{2}}\left(\sec ^{2}(x)\right)^{\frac{2-\nu}{2}}}{4 \pi^{\nu / 2}} 2 \tan ^{2}(x) \int_{0}^{\infty} \int_{0}^{\infty} d u d v x$

$$
x\left(\operatorname{senh}^{2}(v)\right)^{\frac{2-v}{2}}\left(\cos ^{2}(u)-\cos ^{2}(x)\right)^{\frac{2-v}{2}}+
$$




$$
\begin{aligned}
& +2 \sec ^{2}(x) \int_{0}^{\infty} \int_{0}^{\infty} d u d v \operatorname{sen}^{2}(u)\left(\operatorname{senh}^{2}(v)\right)^{2} x \\
& \times\left(\cosh ^{2}(u)-\cos ^{2}(x)\right)^{\frac{2-v}{2}}+2\left(\tan ^{2}(x)^{-1}\right) \int_{0}^{\infty} \int_{0}^{\infty} d u d v x \\
& \times\left(\operatorname{senh}^{2}(v)\right)^{\frac{2-v}{2}}\left(\cosh ^{2}(u)-\cos ^{2}(x)\right)^{\frac{2-v}{2}}
\end{aligned}
$$

Denominando o primeiro, o segundo e o terceiro termos res pectivamente por $A, B$ e $C$ e chamando de a o fator multipli cativo podemos escrever

$$
V(C)=a(A+B+C)
$$

Para calcularmos cada uma das integrais, usamos a expressão $[5.1]:$

$\int_{0}^{\infty} d x \frac{\operatorname{senh}^{\mu-1}(x) \cosh ^{\nu-1}(x)}{\left(\cosh ^{2}(x)-\beta\right)^{\rho}}=\frac{{ }^{F_{1}}\left(\rho, 1+\rho-\frac{\mu+\nu}{2} ; 1+\rho-\frac{\nu}{2} ; \beta\right)}{2 B\left(\frac{\mu}{2}, 1+\rho-\frac{\mu+\nu}{2}\right)}$

2. $\operatorname{Re}(1+0)>\operatorname{Re} v$

$2 \operatorname{Re}(1+\rho)>\operatorname{Re}(\mu+\nu)$.

Com isso obtemos

$$
A=\frac{\tan ^{2}(x) B\left(\frac{3-v}{2}, \frac{v-2}{2}\right){ }_{2} F_{1}\left(\frac{v-2}{2}, \frac{v-2}{2} ; \frac{v-1}{2} ; \cos ^{2}(x)\right)}{2 B\left(\frac{3}{2}, \frac{v-2}{2}\right)},
$$


$B=\frac{\sec ^{2}(x) B\left(\frac{3-v}{2}, \frac{v-2}{2}\right)}{2 B\left(\frac{3}{2}, \frac{v-4}{2}\right)}{ }_{2} F_{3}\left(\frac{v-2}{2}, \frac{v-4}{2} ; \frac{v-1}{2} ; \cos ^{2}(x)\right)$

e

$C=\frac{\left(\tan ^{2}(x)-1\right) B\left(\frac{5-\nu}{2}, \frac{\nu-4}{2}\right)}{2 B\left(\frac{1}{2}, \frac{v-2}{2}\right)} 2_{2} F_{1}\left(\frac{\nu-2}{2}, \frac{\nu-2}{2} ; \frac{\nu-1}{2} ; \cos ^{2}(x)\right)$

O residuo de $V(C)$ no polo $\nu=4$ vem da expressão $C$, isto é

$\underset{V=\Lambda}{\operatorname{Res}(V(C))}=\frac{1}{4 \pi}\left[\frac{\left(\tan ^{2}(x)-1\right)}{\left(2 \sec ^{2}(x)\right)} 2 \mathrm{~F}_{1}\left(1,1 ; \frac{3}{2} ; \cos ^{2}(x)\right)\right]$

como

${ }_{2} F_{1}\left(1,1, \frac{3}{2} ; \cos ^{2}(x)\right)=\frac{\frac{\pi}{2}-x}{\operatorname{sen}(x) \cos (x)}$,

temos

$$
\begin{aligned}
\operatorname{Res}(V(C)) & =\frac{1}{8 \pi^{2}} \cot (x)\left(\tan ^{2}(x)-1\right)\left(\frac{\pi}{2}-x\right)= \\
& =\frac{(\pi-\alpha)}{16 \pi^{2}}\left(\tan \left(\frac{\alpha}{2}\right) \cot \left(\frac{\alpha}{2}\right)\right)=\frac{(\alpha-\pi) \cot (\alpha)}{8 \pi^{2}},
\end{aligned}
$$

onde $\alpha=2 x$.

No jimite de a tendendo para 0, a hipérbole tende para as suas assíntotas. Como o resultado acima independente de a, concluímos que ele é vāilido tambëm para a interseção de suas semiretas, no gauge de Feynman. o mesmo acontecendo com a parte finita. 
A expressão a seguir ê para se obter a par te finjta de $V(C)$. Nos termos que não têm polo a parte fini ta ë obtida fazendo $v$ tender para 4 . No termo que tem o po lo que é o caso de $C$ devemos multiplicā-lo por $v-4$, derivar com relação a $v$ e finalmente fazer $v$ tender a 4 , como segue

$$
\begin{aligned}
& \text { PF }[V(C)]=\frac{1}{4 \pi^{2} \sec ^{2}(x)}\left[\frac{\operatorname{sen}(x)}{2 \cos ^{3}(x)}\left(\frac{\pi}{2}-x\right)\right]+ \\
& +\frac{d}{d \nu}\left(\frac{\left.(\nu-4) 4^{\frac{4-v}{2}} \Gamma\left(\frac{\nu-2}{2}\right)\left(a^{2}\right)^{\frac{4-v}{2}}\left(\sec ^{2}(x)\right)^{\frac{2-\nu}{2}\left(\tan ^{2}(x)-1\right) B\left(\frac{5-\nu}{2}, \frac{\nu-4}{2}\right)}\right) x}{8 \pi^{\nu / 2} B\left(\frac{1}{2}, \frac{v-2}{2}\right)}\right. \\
& \quad \times{ }_{2} F_{1}\left(\frac{v-2}{2}, \frac{\nu-2}{2} ; \frac{v-1}{2} ; \cos ^{2}(x)\right) .
\end{aligned}
$$




\section{CAPITULO 6}

\section{CATLCULO DAS PARTES FINITAS PARA A CIRCUNEERENCTA}

A finalidade deste capitulo è apresentax as partes finitas do loop de wilson quântico, em quarta ordem, para a circunferência em quatro dimensões.

\section{1. - INTEGRAL NECESSÄRTA PARA O CALCULO DOS DIAGRAMAS G Ga, $\mathrm{G}_{4}, \mathrm{G}_{4} \mathrm{C}$ DO CAPTTULO QUATRO}

No capitulo quatro obtivemos os resultados dos cálculos do loop de wilson, em quarta ordem, em função de integrações em um certo contorno. Se escolhermos um con torno simples como a circunferência podemos resolve-las.

Os resultados dos diagramas $G_{4}$ a $G_{4 b}, G_{4 c}$ mos trado nas expressões (4.55), são dependentes da integral

$$
\oint \oint d x^{\sigma} d y^{\rho} \delta_{\sigma \rho}|x-y|^{\sigma-2 v} .
$$

Para integramos na circunferência de raio $R$, $e$ convenien te escrever a posição dos pontos $x$ e $y$ da curva em coorde nadas polares, como segue

$$
\begin{array}{ll}
x^{1}=\operatorname{Rcos}(u), & d x^{1}=-\operatorname{Rsen}(u) d u, \\
x^{2}=\operatorname{Rsen}(u), & d x^{2}=\operatorname{Rcos}(u) d u, \\
y^{l}=\operatorname{Rcos}(v), & d y^{2}=-\operatorname{Rsen}(v) d v, \\
y^{2}=\operatorname{Rsen}(v), & d y^{2}=\operatorname{Rcos}(v) d v,
\end{array}
$$


onde $u$ e $v$ são ângulos que estão mostrados na figura 6.1 .

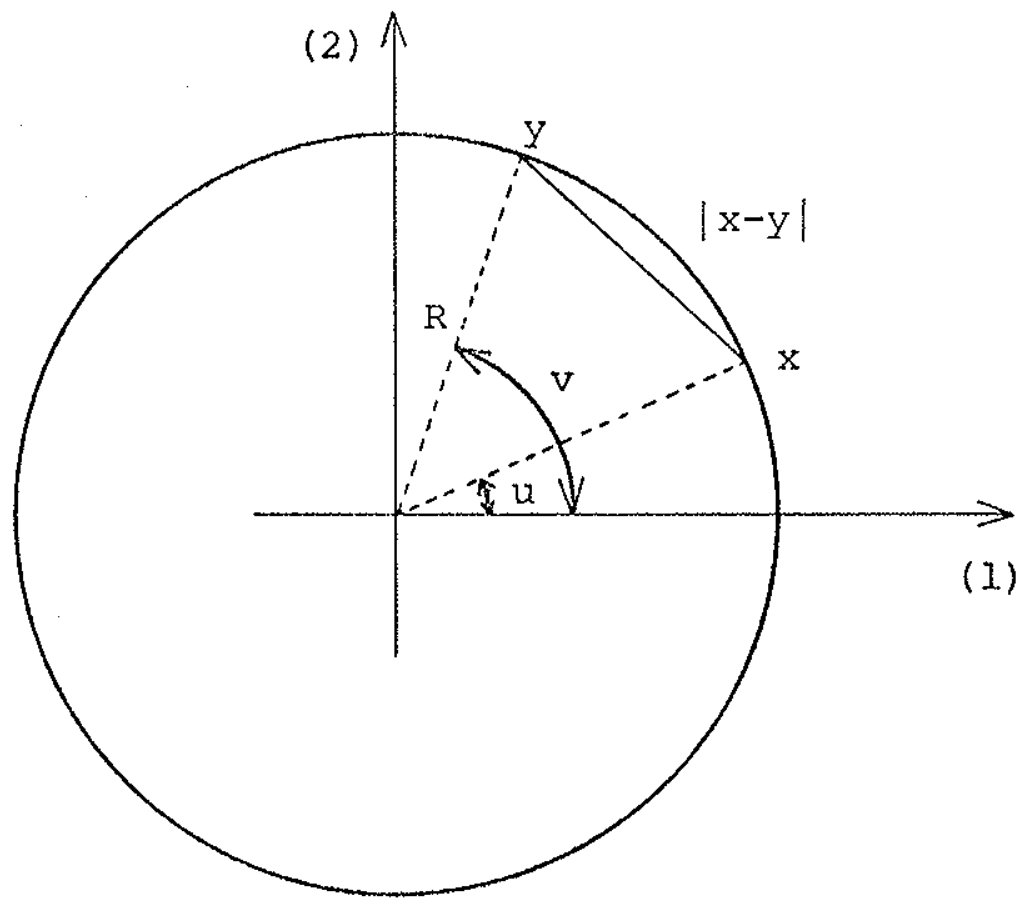

fig. 6.1

Fazendo a contração dos índices, substituindo a igualdade

$$
|x-y|^{2}=4 R^{2} \operatorname{sen}\left(\frac{v-u}{2}\right)
$$

e as relações (6.2), na expressão (6.1) temos

$$
\begin{aligned}
I & =\oint \oint d x^{\circ} d y^{\rho} \delta_{\sigma \rho}|x-y|^{6-2 \nu}=4^{3-\nu}\left(R^{2}\right)^{4-\nu} x \\
& \times \int_{0}^{2 \pi} \int_{0}^{2 \pi} d u d v\left[1-2 \operatorname{sen}\left(\frac{u-v}{2}\right)\right]\left[\operatorname{sen}^{2}\left(\frac{u-v}{2}\right)\right]^{3-\nu} .
\end{aligned}
$$

Fazendo a mudança de variáveis 


$$
\alpha=\frac{u-v}{2}
$$

$e$

$$
\beta=\frac{u+v}{2}
$$

obtemos para o elemento de area

$$
d \alpha d \beta=\frac{3}{2} d u d v
$$

Com essa mudança de vaxiāveis a nova região de integração passa a ser a que está indicala na fig. 6.2

fig. 6.2
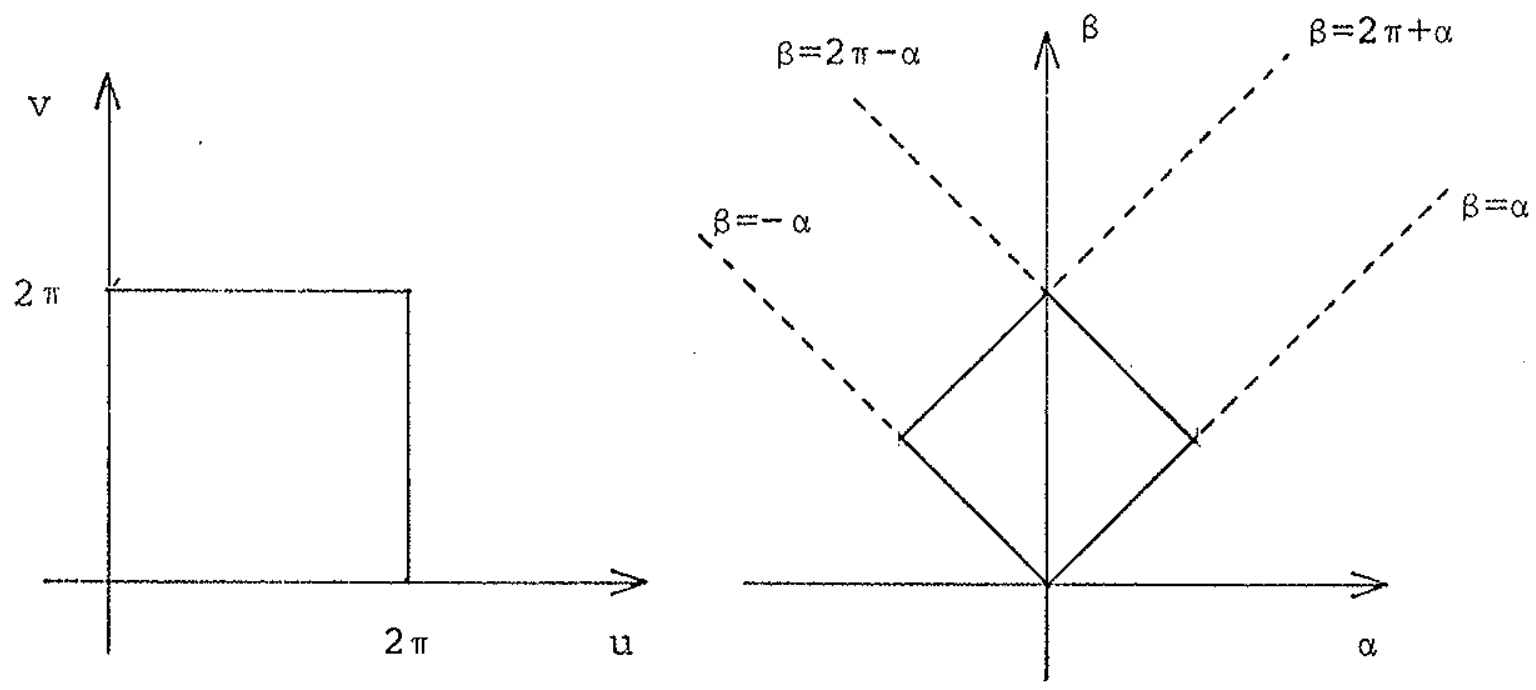

Desta forma a integral fica

$$
I=24^{3-\nu}\left(R^{2}\right)^{4-\nu}\left\{\int_{-\pi}^{0} d \alpha \int_{-\alpha}^{2 \pi+\alpha} d \beta\left[\left(\operatorname{sen}^{2}(\alpha)\right)^{3-\nu}-2\left(\operatorname{sen}^{2}(\alpha)\right)^{4-\nu}\right]+\right.
$$




$$
\left.+\int_{0}^{\pi} d \alpha \int_{\alpha}^{2 \pi-\alpha} d \beta\left[\left(\operatorname{sen}^{2}(\alpha)\right)^{3-\nu}-2\left(\operatorname{sen}^{2}(\alpha)\right)^{l_{1}-\nu}\right]\right\}
$$

ou

$$
I=4^{4-\nu}\left(R^{2}\right)^{4-\nu} \int_{0}^{\pi} d \alpha(2 \pi-2 \alpha)\left(\left(\operatorname{sen}^{2}(\alpha)\right)^{3-\nu}-2\left(\operatorname{sen}^{2}(\alpha)\right)^{1_{5}-\nu}\right) .
$$

Integrando em a utilizando as igualdades [6.1]

$$
\int_{0}^{\pi} d x \operatorname{sen}^{\mu}(x)=\pi^{1 / 2} \frac{\Gamma^{\prime}\left(\frac{\mu+1}{2}\right)}{\Gamma^{r}\left(\frac{\mu+2}{2}\right)}
$$

e

$$
\int_{0}^{\pi} \mathrm{dx} x \operatorname{sen}^{\mu}(\mathrm{x})=\frac{r^{2}}{2^{\mu+1}} \frac{\Gamma(\mu+1)}{\Gamma^{2}\left(\frac{\mu}{2}+1\right)}
$$

temos

$$
\begin{aligned}
I & =4^{4-v}\left(R^{2}\right)^{4-\nu} 2 \pi \pi^{1 / 2}\left[\frac{\Gamma\left(\frac{7-2 \nu}{2}\right)}{\Gamma(4-v)}-2 \frac{\Gamma\left(\frac{9-2 \nu}{2}\right)}{\Gamma(5-v)}\right] \\
& -4^{4-v}\left(R^{2}\right)^{4-v} 2 \pi^{2}\left[\frac{\Gamma(7-2 \nu)}{2^{7-2 \nu} \Gamma^{2}(4-\nu)}-\frac{2 \Gamma^{(9-2 \nu)}}{2^{9-2 \nu} \Gamma^{2}(5-v)}\right] .
\end{aligned}
$$


Utilizando a expressão [6.2]

$$
\Gamma(2 z)=(2 \pi)^{-(1 / 2)} 2^{2 z-(1 / 2)} \Gamma(z) \Gamma\left(z+\frac{1}{2}\right),
$$

temos finalmente

$$
I=\frac{4^{4-v}\left(R^{2}\right)^{4-v} \pi^{3 / 2} \Gamma\left(\frac{7-2 v}{2}\right)(v-3)}{\Gamma(5-v)} .
$$

\section{2 - RESOLUÇÃO DA INTEGRAL DO DIAGRAMA $G_{l^{+}}$SOBRE A CIRCUN \\ - FERENCIA}

Na expressão apresentada nas equações (4.55) para o diagrama $\mathrm{G}_{\mathrm{l}_{\mathrm{g}}} \mathrm{g}$ só contēm a parte divergente; sendo a nossa intenção apresentar uma expressão para a parte fini ta, não podemos partix dessa. O nosso ponto de partida é a expressão (4.25), repetida abaixo,

$$
\begin{aligned}
G_{4}^{\prime} g & =-\frac{n C_{2} g^{4}}{2} \oint d x_{1}^{\mu_{1}} \oint d x_{2}^{\mu_{2}} D_{\mu_{1} \mu_{2}}\left(x_{1}-x_{2}\right) \times \\
& \times \int_{x_{1}}^{x_{2}} d x_{3}^{\mu_{3}} \int_{x_{2}}^{x_{1}} d x_{4}^{\mu_{4}} D_{\mu_{3} \mu_{4}}\left(x_{3}-x_{4}\right) .
\end{aligned}
$$

Esta expressão é a contribuição não-abeliana do diagrama $\mathrm{G}_{4} \mathrm{~g}$. Substituindo o propagador, no espaço de coordenadas dado por (5.1), na expressão acima resulta

$$
G_{4}^{\prime} g=-\frac{n C_{2} g^{4}}{2} \cdot \oint d x_{1}^{\mu_{1}} \oint d x_{2}^{\mu_{2}} D_{\mu_{1} \mu_{2}}\left(x_{1}-x_{2}\right) \times
$$




$$
\begin{aligned}
& \times \int_{x_{1}}^{x_{2}} d x_{3}^{\mu_{3}} \int_{x_{2}}^{x_{1}} d x_{4}^{\mu_{4}} \frac{\Gamma\left(\frac{\nu-2}{2}\right)}{4 \pi \nu / 2} \delta_{\mu_{3} \mu_{4}}\left|x_{3}-x_{4}\right|^{2-\nu}+ \\
& +\frac{n C_{2} g^{4} A \cdot \Gamma\left(\frac{\nu-4}{2}\right)}{32 \pi \nu / 2} \oint d x_{1}^{\mu_{1}} \oint d x_{2}^{\mu_{2}} D_{\mu_{1} \mu_{2}}\left(x_{1}-x_{2}\right) \times \\
& \times \int_{x_{1}}^{x_{2}} d x_{3}^{\mu_{3}} \int_{x_{2}}^{x_{1}} d x_{4}^{\mu_{4}} \partial_{\mu_{3}}^{x_{3}} \partial_{\mu_{4}}^{x_{4}}\left|x_{3}-x_{4}\right|^{4-\nu} .
\end{aligned}
$$

Vamos chamar o primeiro termo da expressão (6.13b) de J. O segundo termo pode ser integrado em $x_{3}$ e $x_{4}$. Com isso obtemos para a expressão (6.13b)

$G_{4}^{\prime} g=J+\frac{\mathrm{nC}_{2} \mathrm{~g}^{4} \mathrm{~A} \Gamma\left(\frac{\nu-4}{2}\right)}{16 \pi^{\nu / 2}} \oint \oint d x^{\mu} d y^{\nu} D_{\mu \nu}(x-y)|x-y|^{4-\nu}$.

ou

$\mathrm{G}_{4 \mathrm{~g}}^{\prime} \equiv \mathrm{J}+\mathrm{K}$

\section{3 - CÃICULO DAS INTEGRAIS DA EXPRESSÃO K}

Substi.tuindo em $\mathrm{K}$ a expressão do propagador obtemos a seguinte expressão

$$
\begin{aligned}
& K=\frac{\mathrm{nC}_{2} \mathrm{~g}^{4} \mathrm{~A} \Gamma\left(\frac{\nu-4}{2}\right) \Gamma\left(\frac{\nu-2}{2}\right)}{64 \pi^{\nu}} \oint \oint d x^{\mu} d y^{\nu}\left\{\delta_{\mu \nu}|x-y|^{2-\nu}-\right.
\end{aligned}
$$

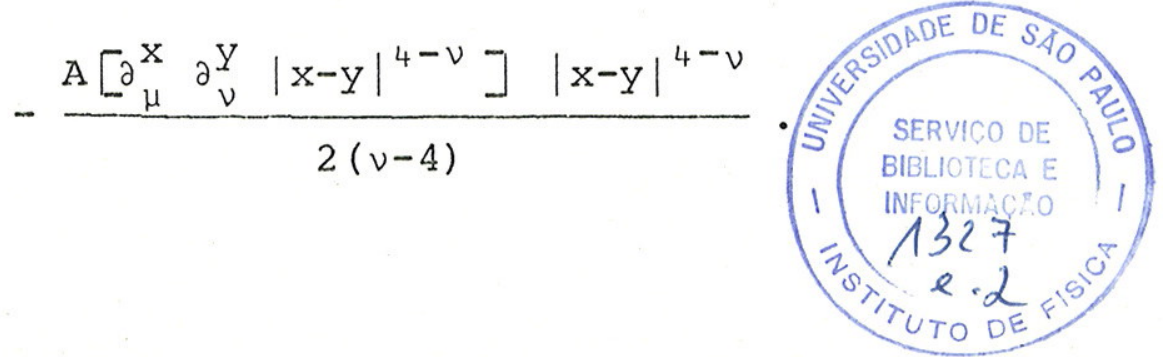


Paxa calcular a integral da expressão $\mathrm{K}$, so bre a circunfexência, é conveniente parametrizar os veto res posição $x$ e $y$. Como podemos escolher o sistema de coor denadas de tal forma que a circunferência esteja centrada na oxigem do plano cartesiano (1) $x$ (2), os vetores posi ção dos pontos na curva podem ser parametrizados como se gue:

$$
x(u)=\operatorname{Rcos}(u) \hat{i}+\operatorname{Rsen}(u) \hat{j}
$$

e

$$
y(v)=\operatorname{RCos}(v) \hat{j}+\operatorname{Rsen}(v) \hat{j}
$$

onde $R$ é o raio da circunferência, $u$ e $v$ são os parâmetros e $\hat{i}, \hat{j}$ são os vetores unitários respectivamente nas dire ções (1) e (2). A figura 6.1 esclaresse melhor o significa do dos símbolos. O primeiro termo da equação (6.16) foi in tegrado na seção 6.1. Substituindo o resultado (6.12) na expressão $(6.16)$ obtemos

$$
\begin{aligned}
& K=\frac{n C_{2} g^{4} \Gamma\left(\frac{v-2}{2}\right) \Gamma\left(\frac{v-4}{2}\right)\left(4 R^{2}\right)^{4-\nu} \pi^{3 / 2} \Gamma\left(\frac{7-2 v}{2}\right)(\nu-3) A}{64 \pi^{v} \Gamma(5-v)}- \\
& -\frac{\mathrm{nC}_{2} g^{4} \Gamma\left(\frac{\nu-2}{2}\right) \Gamma\left(\frac{\nu-4}{2}\right) \AA}{128 \pi^{\nu}(\nu-4)} \oint \oint d x^{\mu} d y^{\nu}\left[\partial_{\mu}^{x} \partial \frac{y}{\nu}|x-y|^{4-\nu}\right] x \\
& x|x-y|^{4-v} .
\end{aligned}
$$

Vamos denominar a integral em (6.18) por K'. Substituindo as relações $(6.17)$ em $\mathrm{K}^{\prime}$, temos

$$
K^{\prime}=\oint \oint d x^{\mu} d y^{\nu}|x-y|^{4-\nu} \partial_{\mu}^{x} \partial_{\nu}^{y}|x-y|^{4-\nu}=
$$




$$
\begin{aligned}
& =\int_{0}^{2 \pi} \int_{0}^{2 \pi} d u d v\left[4 R^{2} \operatorname{sen} 2\left(\frac{u-v}{2}\right)\right]^{\frac{4-v}{2}} \frac{a^{2}}{d u d v} \times \\
& \times\left[4 R^{2} \operatorname{sen}^{2}\left(\frac{u-v}{2}\right)\right]^{\frac{4-v}{2}} .
\end{aligned}
$$

Derivando a expressão acima e reduzindo os termos semelhan tes, obtemos

$$
\begin{aligned}
K^{\prime} & =-\left(4 R^{2}\right)^{4-v} \frac{(v-4)}{4} \int_{0}^{2 \pi} \int_{0}^{2 \pi} d u d v\left[\operatorname{sen}^{6-2 v}\left(\frac{u-v}{2}\right)(v-3)-\right. \\
& \left.-\operatorname{sen}^{8-2 v}\left(\frac{u-v}{2}\right)(v-4)\right] .
\end{aligned}
$$

Fazendo a transformação de variäveis (6.5) e tomando o cui dado de mudar a região de integração como mostra a fijgura 6.2 temos

$$
\begin{aligned}
K^{\prime} & =-(\nu-4)\left(4 R^{2}\right)^{4-\nu} \int_{0}^{\pi} d \alpha \int_{0}^{2 \pi-\alpha} d \beta\left[(\nu-3) \operatorname{sen}^{\varepsilon-2 \nu}(\alpha)-\right. \\
& \left.-(\nu-4) \operatorname{sen}^{8-2 \nu}(\alpha)\right] .
\end{aligned}
$$

Para integrar a expressão anterior, usamos as integrais (6.9) e a fórmula de duplicação das funções gama, com isso obtemos

$$
K^{\prime}=-\frac{(\nu-4) \pi^{3 / 2} \Gamma\left(\frac{7-2 \nu}{2}\right)\left(4 R^{2}\right)^{4-\nu}}{\Gamma(4-\nu)} .
$$


Substituindo esse resultado na expressão $(6.18)$, obtemos finalmente a expressão

$K=\frac{\mathrm{nC}_{2} g^{4} \Gamma\left(\frac{\nu-2}{2}\right) \Gamma\left(\frac{\nu-4}{2}\right) \Gamma\left(\frac{7-2 \nu}{2}\right)\left(4 \mathrm{R}^{2}\right)^{4 \cdots \nu} \mathrm{A}}{64 \pi^{\nu \cdots-\frac{3}{2}} \Gamma(\nu-\nu)}\left(\nu-\frac{A(\nu-4)}{2}\right)$.

6.4 - CALCULO DA EXPERSSÃO J

A expressão J tem a forma

$J=-\frac{n C_{2} g^{4} \Gamma\left(\frac{v-2}{2}\right)}{8 \pi^{\nu / 2}} \oint \oint d x_{1}^{\mu_{1}} d x_{2}^{\mu_{2}} D_{\mu_{1} \mu_{2}}\left(x_{1}-x_{2}\right) \times$

$x \int_{x_{1}}^{x_{2}} d x_{3}^{\mu_{3}} \int_{x_{2}}^{x_{1}} d x_{4}^{\mu_{4}} \delta \mu_{3} \mu_{4}\left|x_{3}-x_{4_{4}}\right|^{2-\nu}$

Analogamente do que fi.cemos na seção anterjor, vamos resol. vex essa integral sobre a circunfexência de raio $R$ centra da na origem do plano cartesiano (1) $x$ (2). Podemos esco Ther a orientação do eixo (1) de tal forma que os pontos $x_{1}$ e $x_{2}$ lhe sejam simétricos. Como está mostrado na fig. 6.3 .

E conveniente fazer uma parametrização analo ga à (6.17),

$$
x_{3}=\operatorname{Rcos}(u) \hat{i}+\operatorname{Rsen}(u) \hat{j}
$$

e

$$
x_{i_{k}}=\operatorname{Rcos}(v) \hat{j}+\operatorname{Rsen}(v) \hat{j}
$$


Para melhor visualização das variäveis mencionadas veja a figura 6.3 .

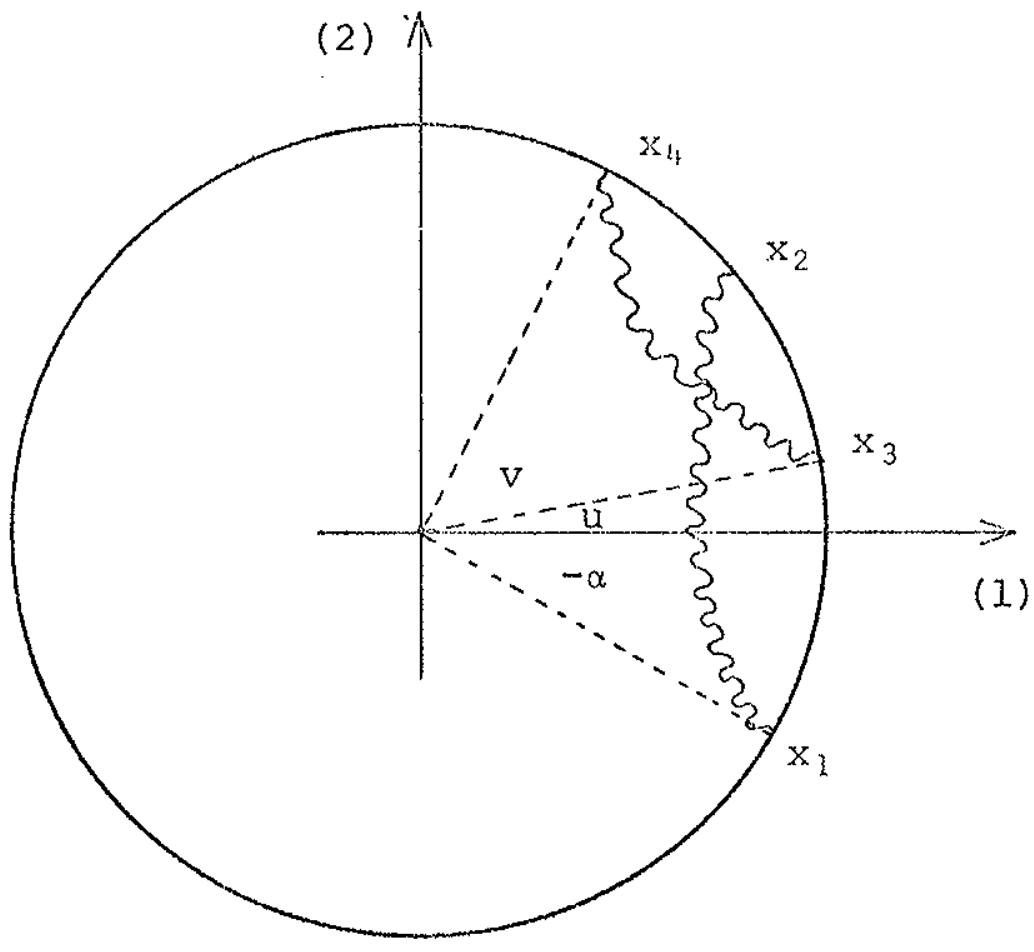

Ej.g. 6.3

Vamos resolver as integrais em $x_{3}$ e $x_{4}$. Seja por definição

$$
J^{\prime} \equiv \int_{x_{1}}^{x_{2}} d x_{3}^{\mu} \int_{x_{2}}^{x_{1}} d x_{4}^{\nu} \delta_{\mu \nu}\left|x_{3}-x_{4}\right|^{2-\nu},
$$

onde o módulo indicado pode ser escrito em função de u e v como segue

$$
\left|x_{3}-x_{4}\right|^{2}=4 R^{2} \operatorname{sen}^{2}\left(\frac{v-u}{2}\right)
$$

A integral $J^{\prime}$ em termos das novas vaxiāveis fica sendo 


$$
\begin{aligned}
J^{\prime}=4^{\frac{2-v}{2}}\left(R^{2}\right)^{\frac{2 . v}{2}} \int_{\alpha}^{2 \pi-\alpha} d v \int_{-\alpha}^{\alpha} d u\left[\operatorname{sen}^{2}\left(\frac{v-u}{2}\right)\right]^{\frac{2-v}{2}} \times \\
\quad \times\left[I-2 \operatorname{sen}\left(\frac{v-u}{2}\right)\right] .
\end{aligned}
$$

Escrevendo os senos da jntegral (6.28) em função da tangen te do arco metade, como segue

$$
\operatorname{sen}\left(\frac{u-v}{2}\right)=\frac{2 \tan \left(\frac{u-v}{2}\right)}{1+\tan ^{2}\left(\frac{u-v}{2}\right)}
$$

e em seguida fazendo

$$
t=\tan \left(\frac{u-v}{2}\right)
$$

e

$$
d u=\frac{4 d t}{3+t^{2}}
$$

temos

$$
\begin{aligned}
J^{\prime}= & 2^{2-v} R^{4-v} \int_{\alpha}^{2 \pi-\alpha} d v\left\{\int_{-\tan \left(\frac{\alpha+v}{4}\right)}^{\tan \left(\frac{\alpha-v}{4}\right)} d u \frac{4 d t}{1+t^{2}}\left[\frac{4 t^{2}}{\left(1+t^{2}\right)^{2}}\right]^{\frac{2-v}{2}}-\right. \\
& \left.-\int_{-\tan \left(\frac{\alpha+v}{4}\right)}^{\tan \left(\frac{\alpha-v}{4}\right)} d u \frac{8 d t}{\left(1+t^{2}\right)^{2}}\left[\frac{4 t^{2}}{\left(1+t^{2}\right)^{2}}\right]^{\frac{4-v}{2}}\right\} .
\end{aligned}
$$

Otl 


$$
\begin{aligned}
J^{\prime} & =6^{6-2 \nu} R^{4-v} \int_{\alpha}^{2 \pi-\alpha} d v\left\{\int_{0}^{\tan \left(\frac{\alpha-v}{4}\right)} d t \frac{\left.\left(t^{2}\right)^{2}\right)^{2-v}}{\left(1+t^{2}\right)^{3-v}+}\right. \\
& +\int_{0}^{\tan \left(\frac{\alpha+v}{4}\right)} d t \frac{\left(t^{2}\right)^{\frac{2-v}{2}}}{\left(1+t^{2}\right)^{3-\nu}}-8 \int_{0}^{\tan \left(\frac{\alpha-v}{4}\right)} d t \frac{\left(t^{2}\right)^{\frac{4-v}{2}}}{\left(1+t^{2}\right)^{5-v}}- \\
& \left.-8 \int_{0}^{\tan \left(\frac{\alpha+v}{4}\right)} \frac{\left(t^{2}\right)^{\frac{4-v}{2}}}{\left(1+t^{2}\right)^{5-v}}\right\} .
\end{aligned}
$$

Resolvendo as integrais acima, temos

$$
\begin{aligned}
& J^{\prime}=2^{5-2 \nu} R^{4-\nu} \int_{\alpha}^{2 \pi-\alpha} d v\left\{\frac{2}{3-\nu}\left[\tan ^{2}\left(\frac{\alpha-v}{4}\right)\right]^{\frac{3-\nu}{2}} \times\right. \\
& \times 2 F_{1}\left(3-v, \frac{3-v}{2} ; \frac{5-v}{2} ;-\tan ^{2}\left(\frac{\alpha-v}{4}\right)\right)+\frac{2}{3-v}\left[\tan ^{2}\left(\frac{\alpha+v}{4}\right)\right]^{\frac{3-v}{2}} \times \\
& \times 2 F_{1}\left(3-v, \frac{3-v}{2} ; \frac{5-v}{2} ;-\tan ^{2}\left(\frac{\alpha+v}{4}\right)\right) \cdots \frac{16}{5-v}\left[\tan ^{2}\left(\frac{\alpha-v}{4}\right)\right]^{\frac{5-v}{2}} \times \\
& \times{ }_{2} \mathrm{~F}_{1}\left(5-v, \frac{5-v}{2} ; \frac{7-v}{2} ;-\tan ^{2}\left(\frac{\alpha-v}{4}\right)\right)-\frac{16}{5-v}\left[\tan ^{2}\left(\frac{\alpha+v}{4}\right)\right]^{\frac{5-v}{2}} \times \\
& \left.\times{ }_{2} F_{1}\left(5-v, \frac{5-\nu}{2} ; \frac{7-v}{2} ;-\tan ^{2}\left(\frac{\alpha+v}{4}\right)\right)\right\}
\end{aligned}
$$

fazendo a mudança de variäveis: 


$$
\begin{aligned}
& t=\tan ^{2}\left(\frac{\alpha-v}{4}\right) \text { em duas delas } e \\
& t=\tan ^{2}\left(\frac{\alpha+v}{4}\right) \text { nas xestantes, }
\end{aligned}
$$

obtemos

$$
\begin{aligned}
& J^{\prime}=2^{7-2 v} \mathbb{R}^{i_{+}-v}\left\{-\frac{1}{3-v} \int_{0}^{\cot ^{2}(\alpha / 2)} \mathrm{dt} \frac{\frac{t^{2}-v}{1+t}}{1+t}\right. \\
& \times{ }_{2} F_{1}\left(3-v, \frac{3-v}{2} ; \frac{5-v}{2} ;-t\right)+\frac{8}{5-v} \int_{0}^{\cot ^{2}(0 / 2)} d t \frac{\frac{4-v}{2}}{1+t} \times \\
& \times{ }_{2} F_{1}\left(5-v, \frac{5-v}{2} ; \frac{7-v}{2} ;-t\right)+\frac{1}{3-v} \int_{\tan ^{2}(\alpha / 2)}^{\infty} d t \frac{t^{\frac{2-v}{2}}}{1+t} \times \\
& \times{ }_{2} \mathrm{~F}_{1}\left(3-v, \frac{3-v}{2} ; \frac{5-v}{2} ;-t\right)-\frac{8}{5-v} \int_{\tan ^{2}(\alpha / 2)}^{\infty} d t \frac{t^{2}}{1+t} \times \\
& \times{ }_{2} \mathrm{~F}_{1}\left(5-v, \frac{5-v}{2} ; \frac{7-v}{2} ;-t\right\}
\end{aligned}
$$

o cîrculo de convergência da sërie hipergeomëtrica

$$
\begin{aligned}
{ }_{2} W_{1}(a, b ; c ; z) & =\sum_{n=0}^{\infty} \frac{(a) n(b) n z^{n}}{(c)_{n}}=\frac{\Gamma(c)}{\Gamma(a) \Gamma(b)} \times \\
& \times \sum_{n=0}^{\infty} \frac{\Gamma(a+n) \Gamma(b+n)}{\Gamma(c+n)} \frac{z^{n}}{n !}
\end{aligned}
$$


é o círculo unitário $|z|=1$. As suas propriedades são:

- divergência quando $R(c-a-b) \leqslant-1$;

- convergência absoluta quanao $R(c-a-b)>0$;

- convergência condicional quando $-1<R(c-a-b) \leqslant 0$, o ponto $z=1$ è excluído.

No caso das integrais (6.36), cujos limites de integr̀ação dependem de $\cot ^{2}(\alpha / 2)$ e $\tan ^{2}(\alpha / 2)$, existem valores de a tal que a integração se extende a regiões nas quais não estão definidas as funções hipergeomëtricas. Para resolver esse problema devemos fazer a continuação analítica da hipergeo métrica para todo o plano complexo. Essa continuação analí. tica é dada por [6.2]

${ }_{2} F_{1}(a, b ; c ; z)=\frac{1}{(1-z)^{a}}{ }_{2} F_{1}\left(a, c-b ; c ; \frac{z}{z-3}\right)$

ou

${ }_{2} F_{1}(a, b ; c ; z)=\frac{1}{(1-z)^{b}}{ }_{2} F_{1}\left(b, c-a ; c ; \frac{z}{z-1}\right)$.

Utilizando ora uma ora outra dessas continuações analiti cas, de tal forma que as hipergeomëtricas resultantes sa tisfaçam a condição de convergência absoluta no limite quan do a dimensão tende para quatro, obtemos

$J^{\prime}=2^{7-2 v} R^{4-v}\left\{-\frac{1}{3-v} \int_{0}^{\cot ^{2}(\alpha / 2)} d t \frac{(t)^{\frac{2-\nu}{2}}}{(1+t)^{4-v}} \times\right.$

$\times{ }_{2} F_{1}\left(3-v, 1 ; \frac{5-v}{2} ; \frac{t}{1+t}\right)+\frac{8}{5-v} \int_{0}^{\cot ^{2}(\alpha / 2)} d t \frac{(t)^{\frac{4-v}{2}}}{(1+t)^{\frac{7-v}{2}}} \times$ 


$$
\begin{aligned}
& \times 2 F_{1}\left(\frac{5-v}{2}, \frac{v-3}{2} ; \frac{7-v}{2} ; \frac{t}{1+t}\right)+\frac{1}{3-v} \int_{\tan ^{2}(\alpha / 2)}^{\infty} d t \frac{(t)^{\frac{2-v}{2}}}{(1+t)^{4-v}} \times \\
& \times{ }_{2} M_{1}\left(3-v, 1 ; \frac{5-v}{2} ; \frac{t}{1+t}\right)-\frac{8}{5-v} \int_{\tan ^{2}(\alpha / 2)}^{\infty} d t \frac{(t) \frac{4-v}{2}}{(1+t) \frac{7-v}{2}} \times \\
& \left.\times{ }_{2} E_{1}\left(\frac{5-v}{2}, \frac{v-3}{2} ; \frac{7-v}{2} ; \frac{t}{1+\tau}\right)\right\}
\end{aligned}
$$

Neste ponto podemos substituir as hipergeomētricas pelos seus desenvolvimentos em sërie, utilizando a expressão $(6.37)$, temos

$$
\begin{aligned}
& J^{\prime}=2^{7-2 \nu} R^{4_{4}-v} \sum_{n=0}^{\infty}\left\{-\frac{\Gamma\left(\frac{5-\nu}{2}\right) \Gamma(3-v+n)}{\Gamma(4-v) \Gamma\left(\frac{5-v}{2}+n\right)} \int_{0}^{\cot ^{2}(\alpha / 2)} d t x\right. \\
& \times \frac{\frac{t^{2+v}}{2}+n}{(1+t)^{4-v+n}}+\frac{\varepsilon}{(5-v) \Gamma\left(\frac{5-v}{2}\right) \Gamma\left(\frac{v-3}{2}\right) \Gamma\left(\frac{7-v}{2}+n\right) \Gamma(n+1)} \times \\
& \times \int_{0}^{\cot ^{2}(\alpha / 2)} d t \frac{t^{\frac{4-v}{2}+n}}{(1+t)^{\frac{7-v}{2}+n}}+\frac{\Gamma\left(\frac{5-v}{2}\right) \Gamma(3-v+n)}{\Gamma(4-v) \Gamma\left(\frac{5-v}{2}+n\right)} \times \\
& \times \int_{\tan ^{2}(\alpha / 2)}^{\infty} d t \frac{\frac{2-v}{2}+n}{(1+t)^{4-v+n}}- \\
& -\frac{8 \Gamma\left(\frac{7-v}{2}\right) \Gamma\left(\frac{5-v}{2}+n\right) \Gamma\left(\frac{v-3}{2}+n\right)}{(5-v) \Gamma\left(\frac{5-v}{2}\right) \Gamma\left(\frac{v-3}{2}\right) \Gamma\left(\frac{7-v}{2}+n\right) \Gamma(n+1)} \times
\end{aligned}
$$




$$
\left.\times \int_{\tan ^{2}(\alpha / 2)}^{\infty} d t \frac{t^{\frac{4-v}{2}+n}}{(1+t)^{\frac{7-v}{2}+n}}\right\} \text {. }
$$

Resolvemos as integrais usando as fórmulas [6.1]

$$
\begin{aligned}
& \int_{0}^{u} d x \frac{x^{\mu-1}}{(1+\beta x)^{\nu}}=\frac{u^{\mu}}{\mu}{ }_{2} F_{1}(\nu, \mu ; 1+\mu ;-\beta u), \\
& \int_{u}^{\infty} d x \frac{x^{\mu-1}}{(1+\beta x)^{\nu}}=\frac{u^{\mu-\nu}}{\beta^{\nu}(\nu-\mu)}{ }_{2} F_{1}\left(\nu, \nu-\mu ; \nu-\mu+1 ;-\frac{1}{\beta u}\right),
\end{aligned}
$$

e obtemos

$$
\begin{aligned}
& J^{\prime}=2^{7-2 \nu} R^{4-\nu} \sum_{n=0}^{\infty}\left\{-\frac{\Gamma\left(\frac{5-\nu}{2}\right) \Gamma(3-\nu+n)}{\Gamma(4-v) \Gamma\left(\frac{5-\nu}{2}+n\right)} \frac{\left(\cot ^{2}(\alpha / 2)\right)^{\frac{4}{2}-v}+n}{\left(\frac{4-\nu}{2}+n\right)} \times\right. \\
& \times{ }_{2} F_{1}\left(4-v+n, \frac{4-v}{2}+n ; \frac{j-v}{2}+n ;-\cot ^{2}(\alpha / 2)\right)+ \\
& +\frac{16 \Gamma\left(\frac{5-\nu}{2}+n\right) \Gamma\left(\frac{\nu-3}{2}+n\right)\left(\cot ^{2}(\alpha / 2)\right)^{\frac{6-\nu}{2}+n}}{\Gamma\left(\frac{v-3}{2}\right) \Gamma\left(\frac{7-v}{2}+n\right) \Gamma(n+1)\left(\frac{6-\nu}{2}+n\right)} \times \\
& \times{ }_{2} \mathrm{~F}_{1}\left(\frac{7-v}{2}+\mathrm{n} ; \frac{6-v}{2}+\mathrm{n} ; \frac{8-v}{2}+\mathrm{n} ;-\cot ^{2}(\alpha / 2)\right)+ \\
& +\frac{\Gamma\left(\frac{5-v}{2}\right) \Gamma(3-v+n)}{\Gamma(4-v) \Gamma\left(\frac{5-v}{2}+n\right)} \frac{\left(\cot ^{2}(\alpha / 2)\right)^{\frac{4-v}{2}}}{\left(\frac{4-v}{2}\right)} \times \\
& \times_{2} \mathrm{~F}_{1}\left(4-v+\mathrm{n}, \frac{4-v}{2} ; \frac{6-v}{2} ;-\cot ^{2}(\alpha / 2)\right)-
\end{aligned}
$$




$$
-\frac{16 \Gamma\left(\frac{5-v}{2}+n\right) \Gamma\left(\frac{v-3}{2}+n\right)}{\Gamma\left(\frac{v-3}{2}\right)} \frac{\left(\cot ^{2}(\alpha / 2)\right) 1 / 2}{\left(\frac{7 \cdots v}{2}+n\right) \Gamma(n+1)}
$$

Tendo integrado $J ' o$ próximo passo ë integrar em $x_{1}$ e $x_{2}$ para determinarmos $J$. A expressão de $J(6.24)$ está repeti da aqui pox comodidade

$$
J=-\cdots \frac{n C_{2} g^{l_{4}} \Gamma\left(\frac{\nu-2}{2}\right)}{8 \pi^{\nu / 2}} \oint \oint d x_{1}^{\mu} d x_{2}^{\nu} D_{\mu \nu}\left(x_{1}-x_{2}\right) J^{\prime}
$$

Convêm salientar que $J^{\prime}$ depende de $x_{1}$ e $x_{2}$ através do ângu lo $\alpha$, pois $\alpha=\frac{v-u}{2}$, onde $u$ e $v$ são respectivamente os parâ metros dos vetores posição $x_{1}$ e $x_{2}$, ou seja

$$
\begin{aligned}
& x_{1}=R \cos (u) \hat{i}+\operatorname{Rsen}(u) \hat{j}, \\
& x_{2}=R \cos (v) \hat{i}+\operatorname{Rsen}(v) \hat{j} .
\end{aligned}
$$

Desnecessário se faz explicar o significado dos símbolos acima, por jā terem sido feitos em outras seções.

Entretanto J em termos desses parämetros re sulta

$$
J=-\frac{\mathrm{nC}_{2} g^{4} \Gamma\left(\frac{\nu-2}{2}\right) \Gamma\left(\frac{\nu-2}{2}\right)}{32 \pi \nu} \int_{0}^{2 \pi} \int_{0}^{2 \pi} d u d v\left\{4^{\frac{2-\nu}{2}}\left(R^{2}\right)^{\frac{4-\nu}{2}} x\right.
$$




$$
\begin{aligned}
& \times\left[1-2 \operatorname{sen}^{2}\left(\frac{u-v}{2}\right)\right]\left[\operatorname{sen}^{2}\left(\frac{u-v}{2}\right)\right]^{\frac{2-v}{2}}-\frac{A}{2(v-4)} \times \\
& \left.\times \frac{d^{2}}{d u d v}\left[4 R^{2} \operatorname{sen}^{2}\left(\frac{u-v}{2}\right)\right]^{\frac{4-v}{2}}\right\} J^{\prime}
\end{aligned}
$$

ou

$$
\begin{aligned}
J & =\frac{n C_{2} g^{4} \Gamma\left(\frac{v-2}{2}\right) \Gamma\left(\frac{v-2}{2}\right)(R)^{4-v} 2^{2-\nu}}{32 \pi^{v}} \int_{0}^{2 \pi} \int_{0}^{2 \pi} d u d v \times \\
& \times\left[1-2 \operatorname{sen}^{2}\left(\frac{u-v}{2}\right)\right]\left[\operatorname{sen}^{2}\left(\frac{u-v}{2}\right)\right]^{\frac{2-v}{2}}+\frac{A}{2}\{(\nu-3) \times \\
& \left.\times\left[\operatorname{sen}^{2}\left(\frac{u-v}{2}\right)\right]^{\frac{2-v}{2}}-(\nu-4)\left[\operatorname{sen}^{2}\left(\frac{u-v}{2}\right)\right]^{\frac{2-v}{2}}\right\} J^{\prime} .
\end{aligned}
$$

Reduzindo os termos semelhantes e escrevendo de uma forma compacta, temos

$$
\begin{aligned}
J & =-\frac{n C_{2} g^{4} \Gamma\left(\frac{v-2}{2}\right) \Gamma\left(\frac{v-2}{2}\right)(R)^{4-v} 2^{2-v}}{32 \pi^{v}}\left[\frac{2+A(v-3)}{2}\right] \times \\
& \times \sum_{a=0}^{1} \int_{0}^{2 \pi} \int_{0}^{2 \pi} d u d v f(A)^{a}\left[\operatorname{sen}^{2}\left(\frac{u-v}{2}\right)\right]^{\frac{2-v}{2}+a} J^{\prime},
\end{aligned}
$$

onde 


$$
E(A)=\frac{4+A(\nu-4)}{2+A(\nu-3)}
$$

Fazendo a muçança de variäveis

$$
\alpha=\frac{\mathrm{v}-\mathrm{u}}{2}
$$

e

$$
\beta=\frac{u+v}{2}
$$

na expressão (6.48), omitindo as passagens por serem anālo gas as feitas anteriormente e integrando em 8 temos

$J=B^{\prime} \sum_{a=0}^{1} \int_{0}^{\pi} d \alpha(\pi-\alpha) f(A)^{a}\left(\operatorname{sen}^{2}(\alpha)\right)^{\frac{2-v}{2}+a} J^{\prime}$,

or. le

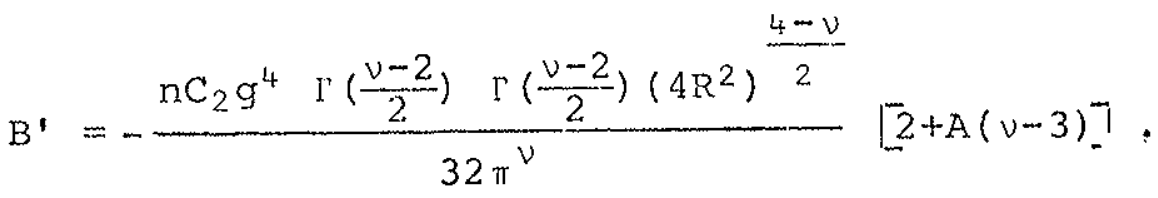

Substituindo J' pela sua expressão resulta

$$
\begin{aligned}
J & =B \sum_{a=0}^{1} \sum_{n=0}^{\infty} \int_{0}^{\pi} d \alpha(\pi-\alpha) f(A){ }^{a}\left[\operatorname{sen}^{2}(\alpha)\right]^{\frac{2-v}{2}+a} \times \\
& \times\left\{\begin{array}{l}
-\frac{\Gamma\left(\frac{5-v}{2}\right) \Gamma(3-v+n)\left[\cot ^{2}(\alpha / 2)\right]^{\frac{4-v}{2}+n}}{\Gamma(4-v) \Gamma\left(\frac{5-v}{2}+n\right)\left(\frac{4-v}{2}+n\right)\left[\cot ^{2}(\alpha / 2)+1\right]^{4-v+n}} \times
\end{array}\right.
\end{aligned}
$$




$$
\begin{aligned}
& \left.x_{2} F_{1}(4-v+n), 1 ; \frac{6-v}{2}+n ; \frac{\cot ^{2}(\alpha / 2)}{1+\cot ^{2}(\alpha / 2)}\right)+ \\
& +\frac{16 \Gamma\left(\frac{5-v}{2}+n\right) \Gamma\left(\frac{v-3}{2}+n\right)\left[\cot ^{2}(\alpha / 2)\right]^{\frac{6-v}{2}+n}}{\Gamma\left(\frac{v-3}{2}\right) \Gamma\left(\frac{7-v}{2}+n\right) \Gamma(n+1)\left(\frac{6-v}{2}+n\right)\left[1+\cot ^{2}(\alpha / 2)\right]^{\frac{6-v}{2}}+n} \times \\
& \times{ }_{2} \mathrm{~F}_{1}\left(\frac{6-\nu}{2}+\mathrm{n}, \frac{1}{2} ; \frac{8-\nu}{2}+\mathrm{n} ; \frac{\cot ^{2}(\alpha / 2)}{1+\cot ^{2}(\alpha / 2)}\right)+ \\
& +\frac{\left.\Gamma\left(\frac{5-v}{2}\right) \Gamma(3-v+n) \cot ^{2}(\alpha / 2)\right|^{\frac{4-v}{2}}}{\Gamma(4-v) \Gamma\left(\frac{5-\nu}{2}+n\right)\left(\frac{4-v}{2}\right)\left[I+\cot ^{2}(\alpha / 2)\right]^{4-\nu+n}} \times \\
& \times{ }_{2} F_{1}\left(4-\nu+n, 1 ; \frac{6-\nu}{2} ; \frac{\cot ^{2}(\alpha / 2)}{1+\cot ^{2}(\alpha / 2)}\right)- \\
& -\frac{16 \Gamma\left(\frac{5-v}{2}+n\right) \Gamma\left(\frac{v-3}{2}+n\right)\left[\cot ^{2}(\alpha / 2)\right]^{1 / 2}}{\Gamma\left(\frac{v-3}{2}\right) \Gamma\left(\frac{7-v}{2}+n\right) \Gamma(n+1)\left(\frac{1}{2}\right)\left[1+\cot ^{2}(\alpha / 2)\right]^{\frac{7-v}{2}}+n} \times \\
& \left.\times{ }_{2} F_{1}\left(\frac{7-v}{2}+n, 1 ; \frac{3}{2} ; \frac{\cot ^{2}(\alpha / 2)}{1+\cot ^{2}(\alpha / 2)}\right)\right\} \text {. }
\end{aligned}
$$

Na expressão acima já foi feito o prolongamento analítico (6.38) ou (6.39) das hipergeométricas da igualdade (6.43). o motivo disso é que a integração em a se extende a regiões onde

$$
\left|-\cot ^{2}(\alpha / 2)\right|>1
$$


nas quais as séxies não estão definidas. A escolha da con tinuação (6.38) ou (6.39) não é arbiträria, pois devemos tomar cuidado para que os parâmetros das hipergeométricas continuadas sejam aqueles que dão convergência à série.

Fazendo a mudança de variáveis,

$$
x=\cot ^{2}(\alpha / 2)
$$

na expressão (6.53) temos

$$
\begin{aligned}
& J=B \sum_{a=0}^{l} \sum_{n=0}^{\infty} \int_{0}^{\infty} d x(\pi-2 \arctan (1 / \sqrt{x})) f(A)^{a} 2^{2-v+2 a} x
\end{aligned}
$$

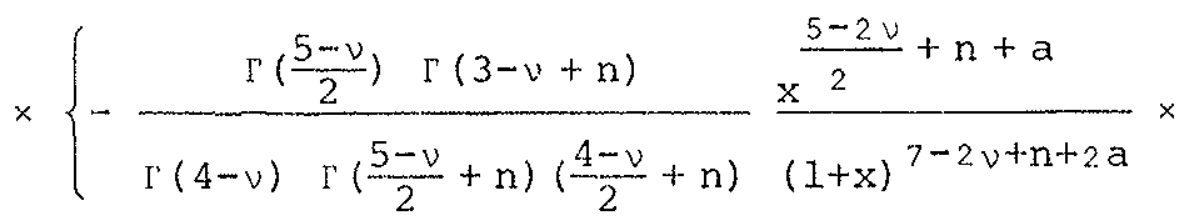

$$
\begin{aligned}
& \times{ }_{2} F_{1}\left(\Delta-v+n, I ; \frac{6-v}{2}+n ; \frac{x}{1+x}\right)+ \\
& +\frac{16 \Gamma\left(\frac{5-v}{2}+n\right) \Gamma\left(\frac{v-3}{2}+n\right)}{\Gamma\left(\frac{v-3}{2}\right) \Gamma\left(\frac{7-v}{2}+n\right) \Gamma(n+1)\left(\frac{6-v}{2}+n\right)} \frac{x^{\frac{7-2 v}{2}+n+a}}{(1+x) \frac{12-3 \nu}{2}+n+2 a} \times \\
& x_{2} F_{1}\left(\frac{5-\nu}{2}+n, \frac{\pi}{2} ; \frac{8-\nu}{2}+n ; \frac{x}{1+x}\right)+ \\
& +\frac{\Gamma\left(\frac{5-v}{2}\right)(3-v+n)}{\Gamma(4-v) \Gamma\left(\frac{5-v}{2}+n\right)\left(\frac{4-v}{2}\right)} \frac{\frac{5-2 v}{2}+a}{(1+x)^{7-2 \nu+n+2 a}} \times \\
& { }_{2} F_{1}\left(4-v+n, 1 ; \frac{6-v}{2} ; \frac{x}{1+x}\right)-
\end{aligned}
$$




$$
\begin{aligned}
& -\frac{16 \Gamma\left(\frac{5-v}{2}+n\right) \Gamma\left(\frac{v-3}{2}+n\right)}{\Gamma\left(\frac{v-3}{2}\right) \Gamma\left(\frac{7-v}{2}+n\right) \Gamma(n+1)\left(\frac{1}{2}\right)} \frac{\frac{2-v}{2}+a}{(1+x) \frac{x^{\frac{3-3 v}{2}}+n+2 a}{2} \times} \times \\
& \left.\times{ }_{2} \mathrm{~F}_{1}\left(\frac{7-v}{2}+n, 1 ; \frac{3}{2} ; \frac{x}{1+x}\right)\right\} .
\end{aligned}
$$

Em seguida daremos o resultado final. omitindo os seguintes passos:

- substituição das hjpergeométricas por suas expansões em sēxie;

- utilizando a identidade paxa o arco cuja tangente é $1 / \sqrt{\mathrm{x}}$

$$
\begin{aligned}
\arctan (x) & =\frac{x}{\sqrt{1+x^{2}}} 2 F_{1}\left(\frac{1}{2}, \frac{1}{2} ; \frac{3}{2} ; \frac{x^{2}}{1+x^{2}}\right)= \\
& =\sum_{n=0}^{\infty} \frac{\Gamma\left(\frac{1}{2}+n\right)}{2 \sqrt{\pi}\left(\frac{1}{2}+n\right) \Gamma(n+1)}\left[\frac{x^{2}}{1+x^{2}}\right]^{n+\frac{1}{2}} ;
\end{aligned}
$$

- em seguida integramos termo a termo utilizando a Eóx mula $[6.1]$

$$
\int_{0}^{\infty} \mathrm{dx} \frac{\mathrm{x}^{\mu-1}}{(1+x)^{\sigma}}=\frac{\Gamma(\mu) \Gamma(\sigma-\mu)}{\Gamma(\sigma)},
$$

obtendo 
$J=B \sum_{a=0}^{l} f(A) 2^{2-v+2 a}\left\{\sum_{n=0}^{\infty} \sum_{m=0}^{\infty} \times\right.$

$\times\left[\frac{\Gamma\left(\frac{5-v}{2}\right) \Gamma(3-v+n) \Gamma\left(\frac{6-v}{2}+n\right) \Gamma(4-v+n+m) \Gamma\left(\frac{7-2 v}{2}+n+m+a\right) \Gamma\left(\frac{7-2 v}{2}+a\right)}{\Gamma(4-v) \Gamma\left(\frac{5-v}{2}+n\right)\left(\frac{4-v}{2}+n\right) \Gamma(4-v+n) \Gamma\left(\frac{6-v}{2}+n+m\right) \Gamma(7-2 v+n+m+2 a)}+\right.$

$+\frac{16}{\sqrt{\pi}} \frac{\Gamma\left(\frac{5-\nu}{2}+n\right) \Gamma\left(\frac{v-3}{2}+n\right) \Gamma\left(\frac{1}{2}+m\right) \Gamma\left(\frac{9-2 v}{2}+n+m+a\right) \Gamma\left(\frac{3-v}{2}+a\right)}{\Gamma\left(\frac{v-3}{2}\right) \Gamma\left(\frac{7-v}{2}+n\right) \Gamma(n+1)\left(\frac{6-v}{2}+n+m\right) \Gamma(m+1) \Gamma\left(\frac{12-3 v}{2}+n+n+2 a\right)}+$

$+\frac{\Gamma\left(\frac{5-v}{2}\right) \Gamma(3-v+n) \Gamma\left(\frac{6-v}{2}+n\right) \Gamma(4-v+n+m) \Gamma\left(\frac{7-2 v}{2}+m+a\right) \Gamma\left(\frac{7-2 v}{2}+m+a\right)}{\Gamma(4-v) \Gamma\left(\frac{5-v}{2}+n\right)\left(\frac{4-v}{2}\right) \Gamma(4-v+n) \Gamma\left(\frac{6-v}{2}+n+m\right) \Gamma(7-2 v+n+m+2 a)}-$

$-16 \sqrt{\pi} \frac{\Gamma\left(\frac{5-v}{2}+n\right) \Gamma\left(\frac{v-3}{2}+n\right) \Gamma\left(\frac{4-v}{2}+m+a\right) \Gamma\left(\frac{9-2 v}{2}+n+a\right) \Gamma\left(\frac{7-v}{2}+n+m\right) \Gamma\left(\frac{9-2 v}{2}+r\right.}{\Gamma\left(\frac{v-3}{2}\right) \Gamma\left(\frac{7-v}{2}+n\right) \Gamma(n+1) \Gamma\left(\frac{7-v}{2}+n\right) \Gamma\left(\frac{13-3 v}{2}+n+m+2 a\right) \Gamma\left(\frac{3}{2}+m\right)}$

$-\sum_{k, n, m=0}^{\infty} \frac{\Gamma\left(k+\frac{1}{2}\right)}{\sqrt{\pi}\left(k+\frac{1}{2}\right) \Gamma(k+1)} \times$

$\times\left[\frac{\Gamma\left(\frac{5-v}{2}\right) \Gamma(3-v+n) \Gamma\left(\frac{6-v}{2}+n\right) \Gamma(4-v+n+m) \Gamma\left(\frac{7-2 v}{2}+n+m+a\right) \Gamma(4-v+a+k)}{\Gamma(4-v) \Gamma\left(\frac{5-v}{2}+n\right)\left(\frac{4-v}{2}+n\right)(4-v+n) \Gamma\left(\frac{6-v}{2}+n+m\right) \Gamma\left(7-2 v+n+m+k+\frac{1}{2}+2 a\right)}+\right.$

$+\frac{1.6 \Gamma\left(\frac{5-v}{2}+n\right) \Gamma\left(\frac{v-3}{2}+n\right) \Gamma\left(\frac{1}{2}+m\right) \Gamma\left(\frac{9-2 v}{2}+n+m+a\right) \Gamma\left(\frac{4-v}{2}+a+k\right)}{\sqrt{\pi} \Gamma\left(\frac{v-3}{2}\right) \Gamma\left(\frac{7-v}{2}+n\right) \Gamma(n+1)\left(\frac{6-v}{2}+n+m\right) \Gamma(m+1) \Gamma\left(\frac{1-3-3 v}{2}+n+m+k+2 a\right)}+$

$+\frac{\Gamma\left(\frac{5-v}{2}\right) \Gamma(3-v+n) \Gamma\left(\frac{6-v}{2}+n\right) \Gamma(4-v+n+m) \Gamma\left(\frac{7-2 v}{2}+m+a\right) \Gamma(4-v+n+k+a)}{\Gamma(4-v) \Gamma\left(\frac{5-v}{2}+n\right)\left(\frac{4-v}{2}\right) \Gamma(4-v+n) \Gamma\left(\frac{6-v}{2}+n+m\right) \Gamma\left(7-2 v+n+m+k+\frac{1}{2}+2 a\right)} \cdots$ 
$\left.\left.-\frac{16 \Gamma\left(\frac{5-v}{2}+n\right) \Gamma\left(\frac{v-3}{2}+n\right) \Gamma\left(\frac{7-v}{2}+n+m\right) \Gamma\left(\frac{4-v}{2}+n+a\right) \Gamma(5-v+n+k+a)}{\Gamma\left(\frac{v-3}{2}\right) \Gamma\left(\frac{7-v}{2}+n\right) \Gamma(n+1) \Gamma\left(\frac{7-v}{2}+n\right) \Gamma\left(\frac{3}{2}+m\right) \Gamma\left(\frac{14-3 v}{2}+n+m+k+2 a\right)}\right]\right\}$.

(6.59)

Na expressäo acjma, observando os limites as sintöticos da razão das funções gama, concluimos que a së rie diverge. O motivo da divergencia pode estar no fato de ter sido fejto vários prolongamentos analiticos, ou que o desenvolvimento em sërie para o cälculo das integrais não seja adequado. Portanto, a intenção original, que era cal cular as partes finitas da circunferencia para qualquer gau ge não foi possivel devido ao diagrama de dois glüons não interagentes. 


\section{CAPTIUIO 7}

\section{CONCLUSÕES}

Nos propusemos a renormalizar a carga da cromo dinâmica quântica até a quarta ordem utilizando o loop de Wil.son quântico com a reqularização dimensional. Grandes simplificações ocorrem para renormalizar a carga com esta tẻcnica. os aiagramas necessários para esse cálculo são so mente os seguintes

$\mathrm{G}_{4 \mathrm{~g}}=$

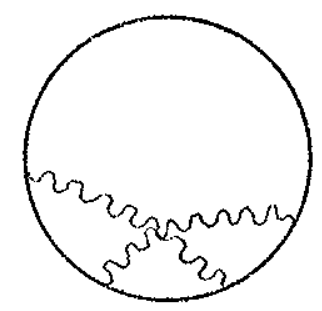

$G_{l C}=$

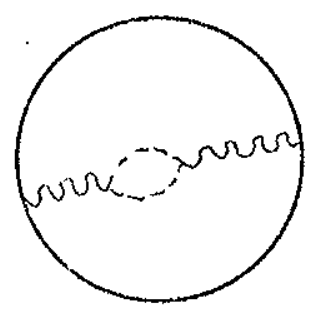

$G_{4 a}=$

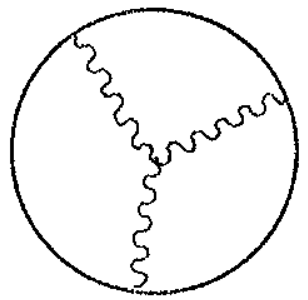

$=\frac{\mathrm{nC}_{2} \mathrm{~g}^{4}}{16 \pi v / 2} \Gamma\left(\frac{\nu-4}{2}\right)\left\{A-\frac{2}{3-\nu}\right\}$

$x \oint \oint d x^{\sigma} d y^{\rho} D_{\sigma \rho}(x-y)|x-y|^{4-\nu}$,

$=\frac{\mathrm{nC}_{2} g^{4} \Gamma^{\prime}\left(2-\frac{\nu}{2}\right) \Gamma^{\prime}\left(\frac{\nu}{2}-1\right) \Gamma\left(\frac{\nu}{2}-1\right) \Gamma(\nu-3)}{2^{8} \pi^{\nu} \Gamma^{\prime}\left(3-\frac{\nu}{2}\right) \Gamma^{\prime}(\nu-2)(\nu-1)} \times$

$x \oint \oint d x^{\sigma} d y^{\rho} \delta_{\sigma \rho}|x-y|^{\sigma-2 \nu}$

$=\frac{n C_{2} g^{4} \Gamma\left(2-\frac{\nu}{2}\right) \Gamma\left(\frac{v}{2}-1\right) \Gamma\left(\frac{v}{2}-1\right) \Gamma(\nu-3)}{2^{6} \pi^{\nu} \Gamma\left(3-\frac{v}{2}\right) \Gamma(\nu-2)} \times$

$\times\left\{\left(2 v-\frac{13}{2}\right) A+A^{2}\left(\frac{v}{4}-1\right)\right\} \times$

$x \phi \phi d x^{\sigma} d y^{\rho} \delta_{\sigma \rho}|x-y|^{\sigma-2 \nu}$, 
$\mathrm{G}_{\mathrm{ib}_{\mathrm{b}}}=$

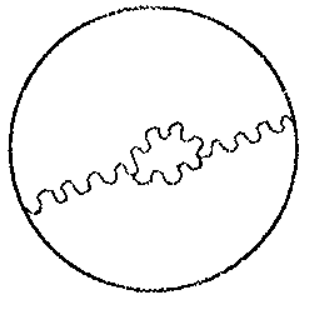

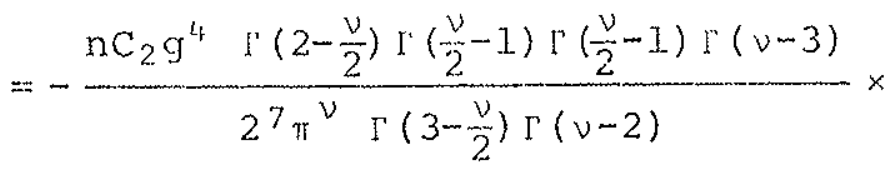

$x\left\{\frac{6 v-5}{2(v-1)}+(2 v-7) A-\left(1-\frac{v}{4}\right) A^{2}\right\} \times$

$x \oint \oint d x^{\sigma} d y^{\rho} \delta_{\sigma \rho}|x-y|^{6-2 \nu}$.

$(4.55)$

ondè $n$ è a dimensão do grupo, $c_{2}$ é a constante de casimir quadrätica, $g$ é a constante de acoplamento, A o fixador do gauge $e D_{o p}(x-y)$ o propagador dos glúons no espaço de coor denadas.

Outra vantagem do método é o de não precisar mos recorrer ao mecanismo de Block e Nordsieck para tratar as àvergências infxavermelhas pois elas não aparecem nesta ordem. Vale ressaltax que o mecanismo de Block e Nordsieck envolve cálculos não triviais. O resultado obtido para a re normalização mencionaà em quatro dimensões é o seguinte

$$
g^{2}=\mu^{v-4} g_{0}^{2}\left\{1+\frac{21 C_{2} g_{0}^{2} \mu^{v-4}}{24 \pi^{2}(4-v)}\right\} .
$$

Este resultado foi obtido por Dotsenko e vergeles [4.I] uti lizando os cálculos de politzer [7.1] para a cromodinâmica quântica, cujas integrais foram regularizadas utilizando-se os parâmetros de corte (cutoff). Dotsenko e vergelles usa ram a prescrição $\frac{1}{4-v}=I_{s} \frac{\Lambda}{\rho}$, onde $\Lambda$ é o parâmetro de corte - o ponto de normalizaçãa no espaço de momenta, para pas sar de una regularização para a outra. Entretanto no traba 
Tho deles não ficou claro se o resultado vale em $v \neq 4$. Pude mos mostrar atraves do nosso trabalho que os resultados dos diagramas ficam dependentes da integral

$$
\oint \oint d x^{\sigma} d y^{\rho} \delta \delta_{\sigma \rho}|x-y|^{6-2 \nu}
$$

Somente em quatro dimensões è que podemos escrever.

$$
\oint \oint d x^{\sigma} d y^{\rho} \delta_{\sigma \rho}|x-y|^{6-2 \nu}=\oint \oint d x^{\sigma} d y^{\rho} D_{\sigma \rho}(x-y) .
$$

Em dimensões diferente de quatro, portanto, a tëcrica do loop de Wilson para a renormalização, merece maior atenção. Em duas dimensōes divergências adicionais ocorrem pois o pró prio propagador tem uma divergência em $\nu=2$, como se pode ob servar abaixo

$$
D_{\sigma \rho}(x-y)=\frac{\Gamma\left(\frac{\nu-2}{2}\right)}{4 \pi^{\nu / 2}}\left\{\delta_{\sigma \rho}|x-y|^{2-\nu}-\frac{A \partial_{\sigma}^{x} \partial_{\rho}^{y}|x-y|^{L^{4}-\nu}}{2(\nu-4)}\right\} \text {. }
$$

Portanto em duas dimensões devemos fazer todos os cálculos usando a parte finita do propagador.

$\mathrm{Na}$ obtenção dos resultados anteriores, em vir tude da complexidade dos cálculos, houve necessidade de nos familiarizarmos com as linguagens simbolicas SCHOONSCHIP e REDUCE2, que nos foram recomendadas em hora oportuna pelo profiessor J.A. Mignaco. 
Outra contribuição nossa foi mostrar que o re siduo obtido do cálculo do fator de fase quântico em quatro dimensöes para uma curva aberta, não depende nem dessa nem do axco como se pode observar na expressão

$$
\begin{aligned}
\operatorname{Res}(V(C)) & =\lim _{\nu \rightarrow 4} \frac{(\nu-4) 2^{8-2 \nu} \Gamma\left(\frac{\nu-2}{2}\right) R^{4-v}\left[\tan ^{2}(\alpha / 2)\right]^{\frac{4}{2}}}{4 \pi^{\nu / 2}(3-v) \frac{4-\nu}{2}}= \\
& =\frac{1}{2 \pi^{2}} .
\end{aligned}
$$

O loop de Wilson é uma técnica nova com a qual pretendemos entendex melhor o fenômeno do confinamento. gual quer callculo que se faça com o loop de wilson na tentativa de nos familiaxizarmos com ele, e justificável. Foi com es ta intensão que nos propusemos tambēm a dax continuidade aos trabalhos de Giambiagi, Bollini e Abud [4.3], nos problemas mencionados a seguix.

Mostramos que dado o ângulo de intersecção de duas semiretas é possivel calcular o residuo e a parte fini ta do fator de fase quântico para esse caso particular, uti lizando uma famjilia de hipérboles, que têm por assintotas essas semiretas. A equação da hipérbole é dada por

$$
\frac{x_{x}^{2}}{a^{2}}-\frac{r^{2}}{b^{2}}=1
$$

se variarmos convenientemente os parâmetros a e b de tal for ma e mantexmos constante a vazão b/a, que é a metade do àn guilo formado pelas semiretas, iremos obtendo a citada fami lita de hipéxboles que vai se transformando no encontro das duas semiretas a medida que fazemos a $\rightarrow 0$. O resultado obtido para o resíduo é 


$$
\operatorname{Res}[V(C)]_{\nu=4}=\frac{(\alpha-\pi) \cot (\alpha)}{8 \pi^{2}}
$$

onde $\alpha$ é o angulo formado pelas semiretas. Este resujtado está consistente com o obtido no trabalho de Giambiagj, Bollini e Abud a menos de diferenças por causa da escolha do gauge, que no nosso caso foi feito no gauge de Feynman e no daqueles no gauge dimensional. A parte finita pode ser obtida utilizando-se a expressão

$$
\begin{aligned}
& P F[V(C)]=\frac{1}{4 \pi^{2} \sec ^{2}(x)}\left[\frac{\operatorname{sen}(x)}{2 \cos ^{3}(x)}\left(\frac{\pi}{2}-x\right]+\right. \\
& +\frac{d}{d v}\left[\frac{(v-4) 4^{\frac{4-v}{2}} \Gamma\left(\frac{v-2}{2}\right)\left(a^{2}\right)^{\frac{4-v}{2}}\left(\sec ^{2}(x)\right)^{\frac{2-\nu}{2}}\left(\tan ^{2}(x)-1\right) B\left(\frac{5-v}{2}, \frac{v-4}{2}\right)}{8 \pi^{v / 2} B\left(\frac{1}{2}, \frac{v-2}{2}\right)} \times\right.
\end{aligned}
$$

$$
\left.\times{ }_{2} F_{1}\left(\frac{\nu-2}{2}, \frac{v-2}{2} ; \frac{\nu-1}{2} ; \cos ^{2}(x)\right)\right]_{v=4}
$$

No último capitulo tivemos a idéia de calcular a parte finita do loop de Wilson para a circunferência, em quarta ordem em quatro dimensöes. Podemos observar nas equa ções (4.55), repetidas nesse capitulo, que as integrais $G_{4 a}, G_{4 b}, G_{4 c^{\prime}}$ (soluções exatas para os diagramas) dependem da integral

$$
I=\oint \oint d x^{\sigma} d y^{\rho} \delta_{\sigma \rho}|x-y|^{6-2 \nu}=\frac{4^{4-\nu}\left(R^{2}\right)^{4-\nu} \pi^{3 / 2} \Gamma\left(\frac{7-2 \nu}{2}\right)(\nu-3)}{\Gamma(5-\nu)},
$$


cujo lado direito foi obtido no capitulo seis. Desta forma é possłvel calcular as partes finitas desses diagramas. Pa ra obtexmos a parte finita do diagrama dos glíons não inte ragentes precisamos recorrex a expressäo (6.13a), repetida a seguix

$$
\begin{aligned}
G_{49}^{\prime} & =-\frac{{n C_{2} g^{4}}_{2}}{2} \oint d x_{1}^{\mu} \int_{1} d x_{2}^{\mu_{2}} D_{\mu_{1} \mu_{2}}\left(x_{1}-x_{2}\right) \int_{x_{1}}^{x_{2}} d x_{3}^{\mu_{3}} \int_{x_{2}}^{x_{1}} d x_{4}^{\mu_{4}} x \\
& \times D_{\mu_{3 \mu_{4}}}\left(x_{3}-x_{4}\right)
\end{aligned}
$$

A expressão $G_{4 g}$ das equações (4.55) não pode ser usada pois ela só contém a parte divergente da integral acima. G' foj. calculada no capítulo seis. Observando a razão das funções gama da expressão (6.59), que no limite de grandes argumen tos ela se comporta como abaixo

$$
\lim _{n \rightarrow \infty} \frac{\Gamma(a+n)}{\Gamma^{*}(b+a)} \sim \frac{1}{n^{b-a}}
$$

onde n é uma variävel e b e a constantes, concluimos que a serie diverge. Desta forma não conseguimos isolar a divex gência como polos no plano complexo da dimensäo. Ficando as sim impossibilitados para calcular a parte finita paraodia grama de dois glúons não interagentes e portanto a circunfe rência em quarta ordem. A sërie deveria sex somada para se obter una função analitica da dimensão coisa que não se con segue devido a complexidade do termo geral, vide expressão $(6.59)$. 


\section{REFERENCIAS BIBIJTOGRAF'ICAS}

[0.1] STRUBBe, H. - Manual for SCHOONSCHIP. Comput. Phys. Commur. V.8:1, 1974 .

[1.l] WETI, H., Ann a. Physjk. V.59:101, 1919.

[1.2] WEYL, H., H., Z.E. Phys. V.56:330, 1929.

[1.3] BolitinNJ, C.G.; GIAMBIAGI, J.J. - Teorias de campos de gauge. Escala de Verão, Universidade de são Car los, 1978.

[L.4] ABERS, S.; LEE, W. - Gauge Theories. Phys. Rep. $90(1), 2973$.

[1.5] FAJDEEV, L.D.; SLAVNOV, A.A. - Gauge fields: Int. to quantum theory. Trad. D.B. Pontecorvo. London, Benjamin/Cummings Publishing, 1980. p. 227.

[1.6] HUANG, Kerson - Quarks leptons \& gauge fields. Singapore, World Scientific Publishing, 1982.

[1.7] YANG, C.N.; MILLS, R.J., Phys. Rev. V.96:191, 1954.

[1.8] AHARONOV, V., BOHM, D., Phys. Rev. V.115:485, 1959.

[1.9] CHAMBERS, R.G., Phys. Rev. Lett. V.5:3, 1960.

[1.10] WIISON, K., PhYS. Rev.V.DI0:2445, 1974.

[1.J1] MAKEENKO, Y.M.; MIGDAL, A.A., Nucl. Phys. B. V.188: $269,1.981$.

[1.12] MIGDAl, A.A., Ann Phys., V.126:279; 290, 1980.

[1.1.3] KOGUT, John B., Rev. Mod. 55(3): 775-836, 1.983.

[2.I] BOLTINI, C.G.; GIAMGIAGT, J.J., Nuova Cimento B $12(1): 20-26,1972$.

[2.2] 't HOOFT, G.; VELTMAN, M., Nucl. Phys. B V.44:1.89, 1.972 .

[2.3] BOLITNI, C.G.; GIAMBIAGI, J.J.; GONZALES, A.D., Nuovo Cimento V.31:550, 1964.

[2.4] CAPPER, D.M.; LEIBBRANDT, G., J. Math. Phys. V.15: $82, \quad 1974$.

CAPPER, D.M.; LEIBBRANDT, G., J. Math. Phys. V.15: $86,1974$.

[2.5] PELBOURGO, R.; AKYCAMPONG, D.A., Nuovo Cimento V. ].9A: 219,1974 . 
[3.1] 't HOOGT, G.; VELTMAN. - Diagrammar. CRRN 73-9, 1973.

[3.2] ROMAN, P. - Introduction to quantum field theory. New York, John Wiley and Sons, 1969.

[3.3] IURIE, D. - Paxticles and fields. New York, Interscience Publj.shers, 1968.

[4.1] DOTSENKO, V.S.; VERGELES, S.N., NuCl. PhyS. B, V.169: 527,1980 .

[4.2] BRANDT, R.A.; NERI, F.; SATO, M., PhYs. ReV. D 24(4): 879,1981 .

[4.3] ABUD, M.; BOLLINT, C.G.; GIAMBIAGI, J.J., Nucl. Phys. B V. 204:109-117, 1982 .

[6.1] GRADSHIEYN, I.S.; RYZHIK, I.M. - Table of integrals, series and products. ed. Alan Jeffrey. 4. ed. aum. New York, Academic Press, 1981. p. 1160.

[6.2] ABRAMOWIIZ, M.; STUGUN, Irene A. ed. - Handbook of mathematical functions. 9. ed. New York, Dover Publications, 1972 . 


\section{APENDICE A}

\section{ALGUMAS INTEGRAIS UTEIS}

Apresentamos aquj algumas integrais que foram usadas no trabalho.

A integral abaixo pode ser calculada usando-se os parâmetros de Feymman

$$
\begin{aligned}
\int \bar{a}^{\nu} p \frac{1}{\left[(p+s)^{2}\right]^{\alpha}\left[p^{2}\right]^{\beta}} & =\frac{\pi^{\nu / 2}\left(s^{2}\right)^{\frac{\nu}{2}-\alpha-\beta} \Gamma\left(\alpha+\beta-\frac{\nu}{2}\right) \Gamma\left(\frac{\nu}{2}-\beta\right) \Gamma\left(\frac{\nu}{2}-\alpha\right)}{\Gamma(\alpha) \Gamma(\beta) \Gamma(\nu-\alpha-\rho)}= \\
& =J(\alpha, \beta, \nu, s)
\end{aligned}
$$

As integrais seguintes podem sex calculadas a partir de A.I. por derivação com relação a s, ou se utilizando dos parâme txos de Feymman

$\int d^{\nu} p \frac{p_{\lambda}}{\left[(p+s)^{2}\right]^{\alpha}\left[p^{2}\right]^{\beta}}=-\left(\frac{\nu}{2}-\beta\right) \frac{s_{\lambda}}{\nu-\alpha-\beta} J(\alpha, \beta, \nu, s)$

$$
\begin{aligned}
& \int d^{\nu} p \frac{p_{\lambda} p_{\rho}}{\left[(p+s)^{2}\right]^{\alpha}\left[p^{2}\right]^{\beta}}=\left(\frac{\nu}{2}-\right) \frac{J(\alpha, \beta, \nu, s)}{(\nu-\alpha-\beta)(\nu-\alpha-\beta+1)} \times
\end{aligned}
$$

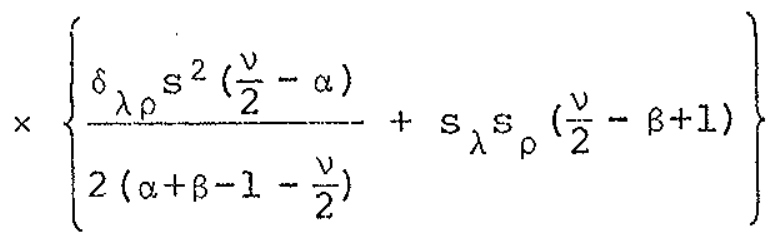




$$
\begin{aligned}
& \int d^{\nu} p \frac{p_{\lambda} p_{\rho} p_{\sigma}}{\left[(p+s)^{2}\right]^{\alpha}\left[p^{2}\right]^{\beta}}=-\frac{\left(\frac{\nu}{2}-\beta+1\right)\left(\frac{\nu}{2}-\beta\right) J(\alpha, \beta, \nu, s)}{(\nu-\alpha-\beta+2)(\nu-\alpha-\beta+1)(\nu-\alpha-\beta)} \times \\
& \times\left\{\frac{s^{2}\left(s_{\lambda} \delta_{\rho \sigma}+s_{\rho} \delta_{\lambda \sigma}+s_{\sigma} \delta \delta_{\rho}\right)\left(\frac{\nu}{2}-\alpha\right)}{2\left(\alpha+\beta-1-\frac{\nu}{2}\right)}+s_{\lambda \rho_{\rho} s_{\sigma}\left(\frac{\nu}{2}-\beta+2\right)}\right\}
\end{aligned}
$$

Para o calculo das integrais anteriores foram usadas as in tegrais

$$
\int d \Omega(v)=\frac{2 \pi^{v / 2}}{I^{\prime}\left(\frac{v}{2}\right)}
$$

onde $\Omega(v)$ e a ärea da superfícje de uma hiperesfera de $v$ di mensões

$$
\int_{0}^{\infty} d p \frac{p^{\nu-1}}{\left(p^{2}+m^{2}\right)^{\alpha}}=\frac{\Gamma\left(\alpha-\frac{v}{2}\right) \Gamma\left(\frac{v}{2}\right)}{2\left[m^{2}\right]^{\alpha-\frac{\nu}{2}} \Gamma^{\prime}(\alpha)}
$$

G.R. pag. 292

$$
\begin{aligned}
& \int a^{\nu} p \frac{1}{\left[\underline{p}^{2}+m^{2}\right]^{\alpha}}=\frac{\pi^{\nu / 2} \Gamma\left(\alpha-\frac{\nu}{2}\right)}{\left[m^{2}\right]^{\alpha-\frac{\nu}{2}} \Gamma(\alpha)} \\
& \int a^{\nu} s\left(s^{2}\right)^{\lambda} e^{i s\left(x_{2}-x_{1}\right)}=\frac{2^{2 \lambda+\nu} \pi^{\nu / 2} \Gamma^{\prime}\left(\lambda+\frac{\nu}{2}\right)}{\Gamma(-\lambda)\left|x_{2}-x_{1}\right|^{2 \lambda+\nu}}
\end{aligned}
$$




\section{CALCULO DO DIAGRAMA DE UM VERTICE COM TRES GLUONS}
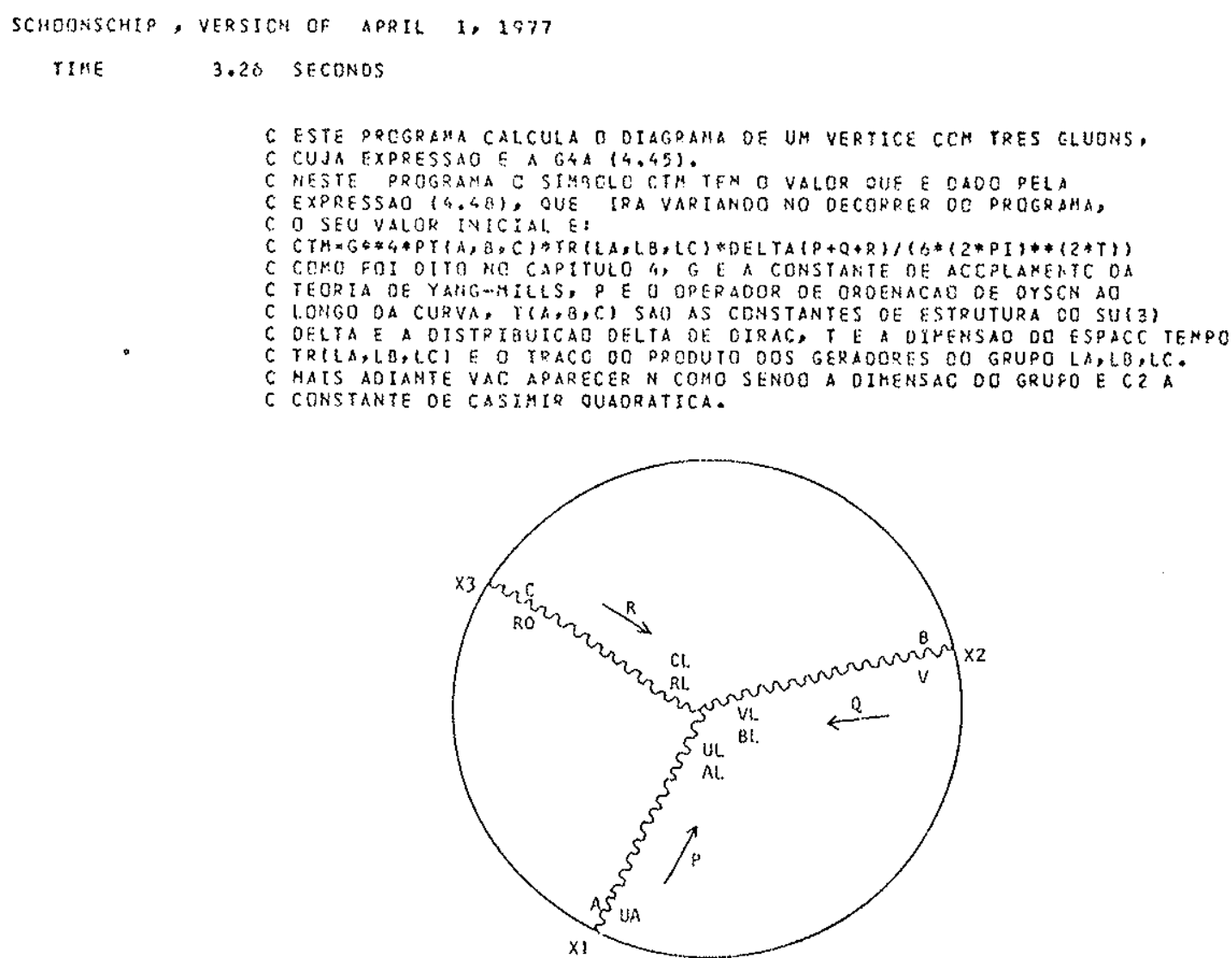

I $U L=T, V L=Y, R L * Y, U * T, V * T, R O=T$

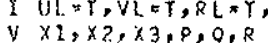

$V \times 2, X 2, \times 3,9,0, R$
$\bar{r} E \times P, G 2,0 G 2, E X P S$

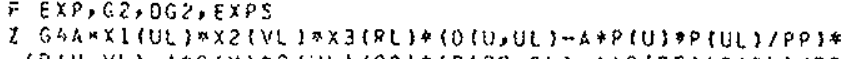

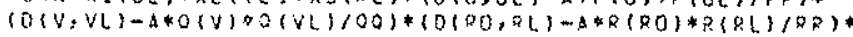

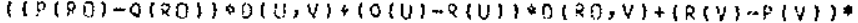

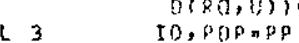

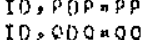

ID, $P 0 R * Q R$

\& C.TH

PEITIT NLIST

PQDit NSTATISTICS

PRIMT CUTPUT

WY $Y$ E:

64A

$+\operatorname{EXP}(0, x 2) * E \times P(0, \times 2) * E \times P(R, \times 3) * C T \times$ 


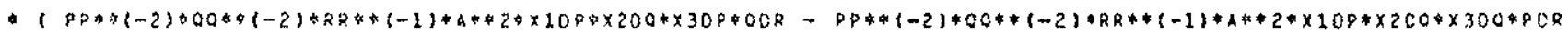

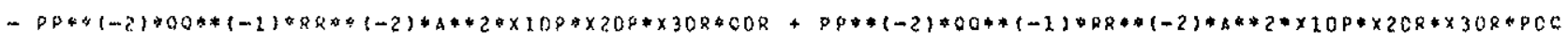

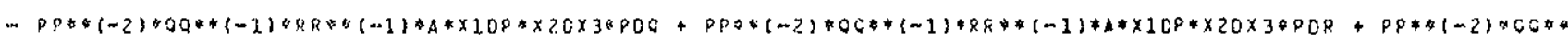

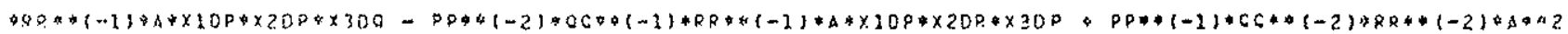

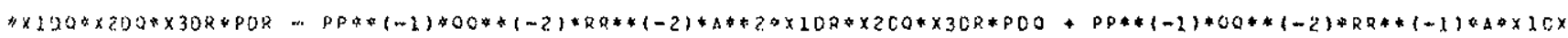

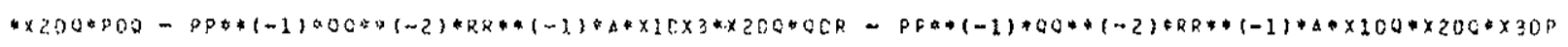

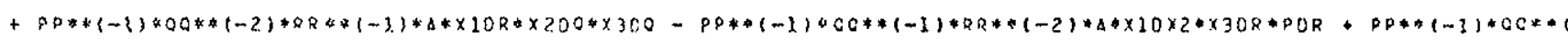

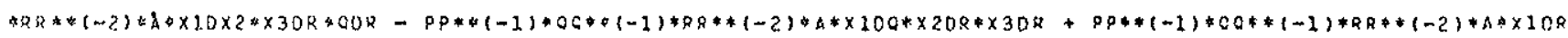

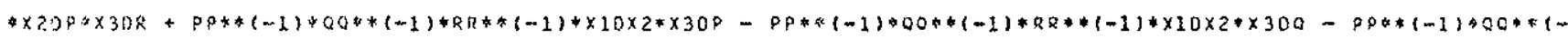
$40 Q * *(-1) * 10 \times 3 * \times 20 \rho+P Q * *(-1) * 00 * *(-1) * R R *(-1) * \times 10 \times 3 * \times 20 R+P Q * *(-1) * 0 C * *(-1) * R R * *(-1) * \times 100 * \times 20 \times 3$ $-P 0 * 4(-1) * 00 *(-1) * 82 *(-1) * 108 * 20 \times 3)+0$.

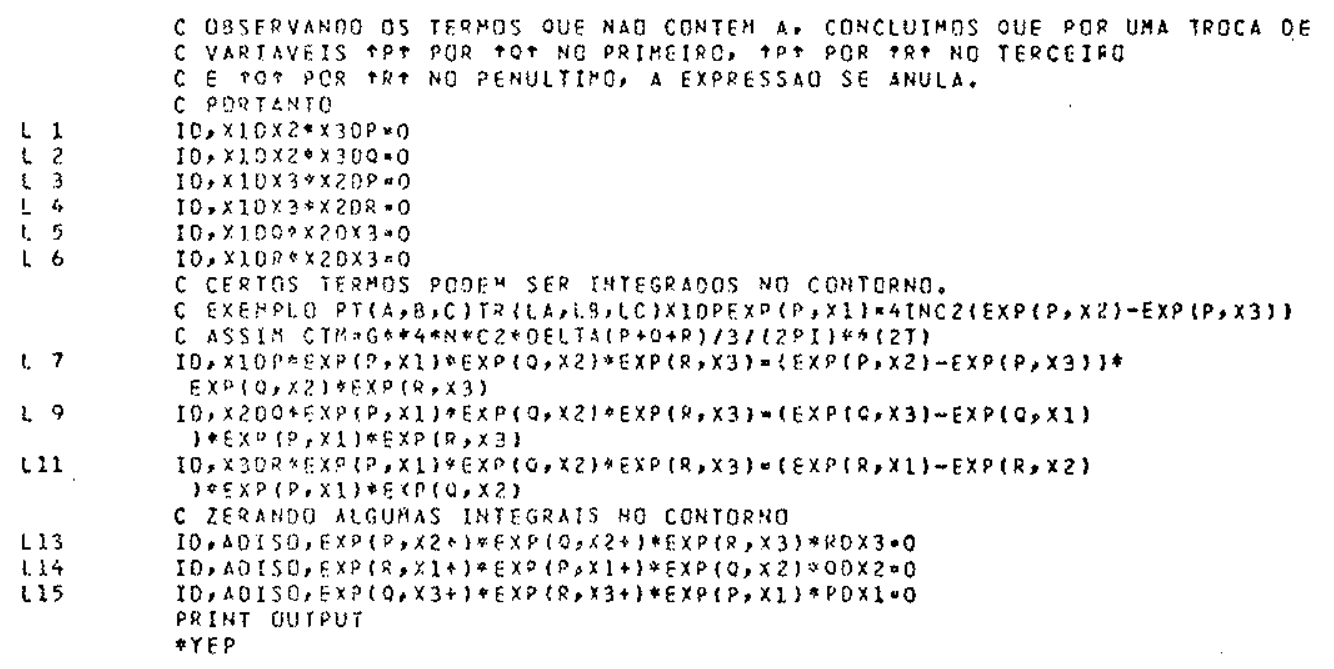

GSA $=$

$+E \times P(P, \times 2) \times E \times P(0, \times 2) * E \times P(R, \times 3) * C T H$

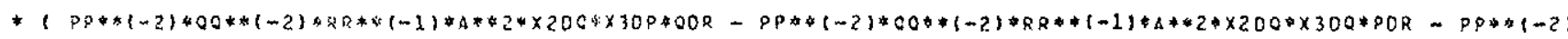

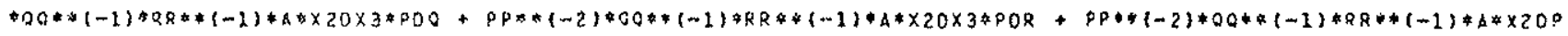

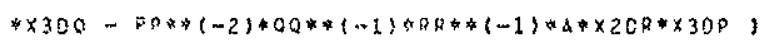

$+E X P(P, \times 3) * E \times P(0, \times 2) * E \times P(R, \times 3) * C T H$ 


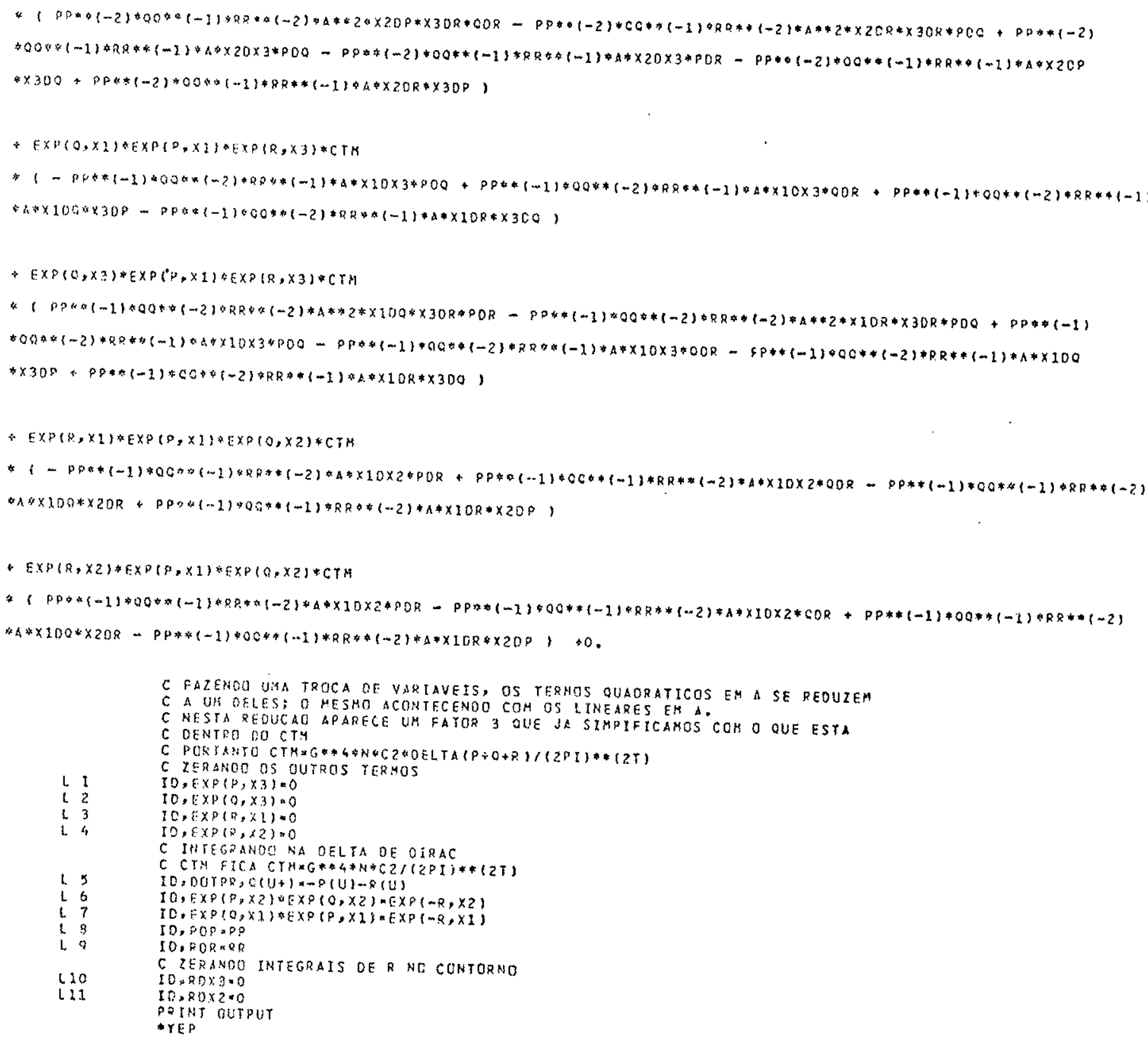




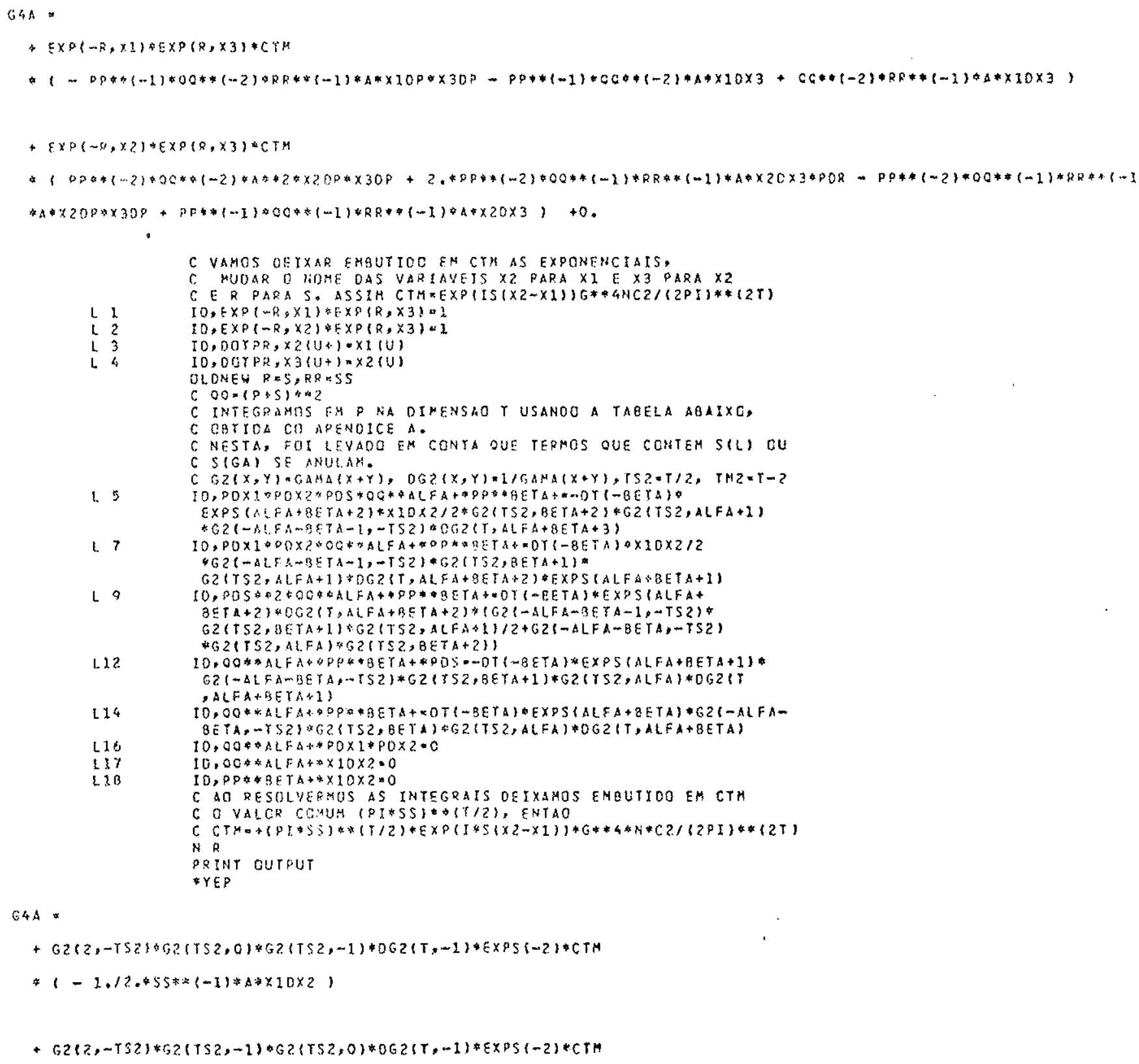




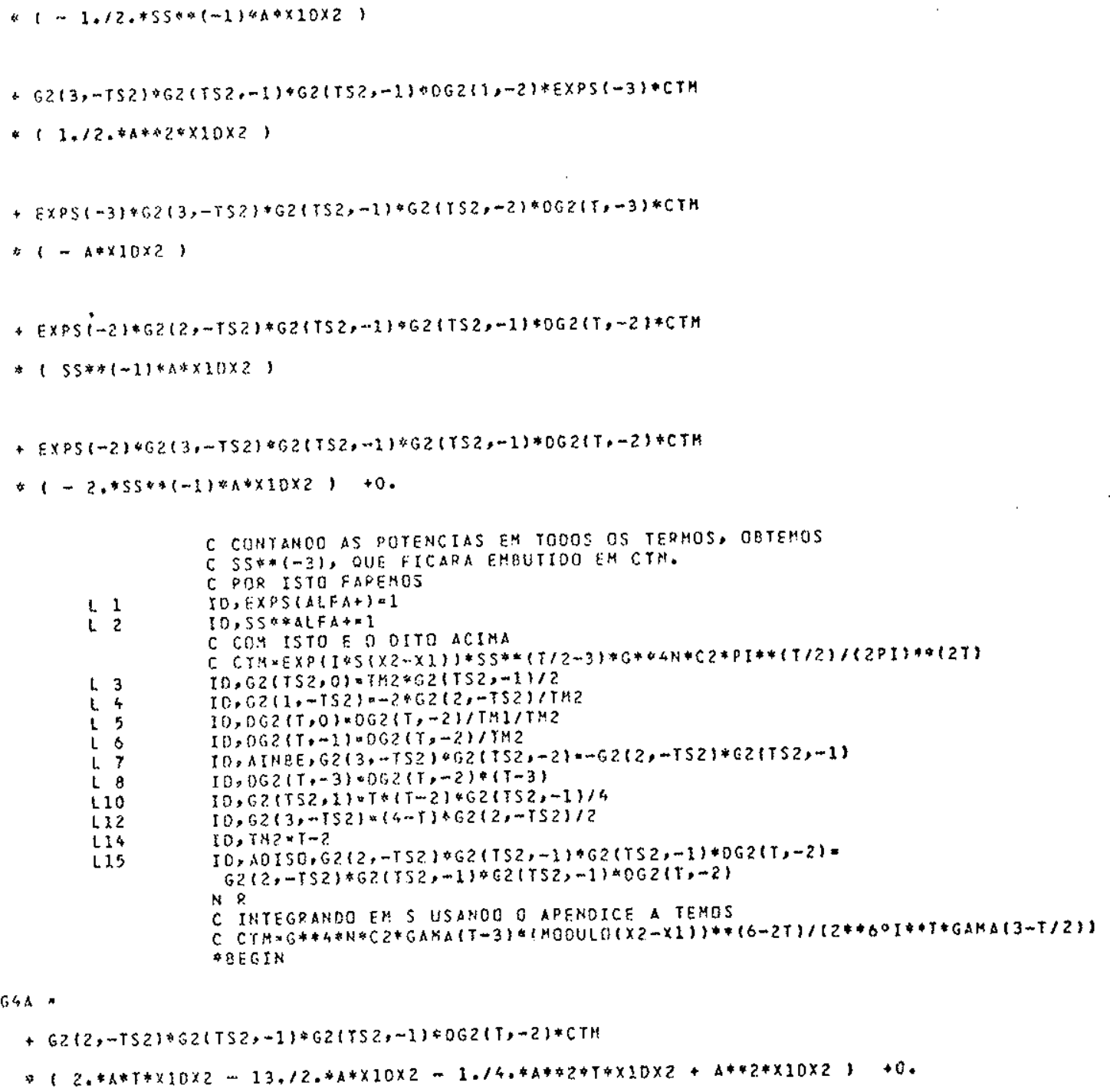




\section{APENDTCE C}

\section{CAILCULO DO DIAGRAMA DE DOIS VERTICES COM TRES GLOONS}

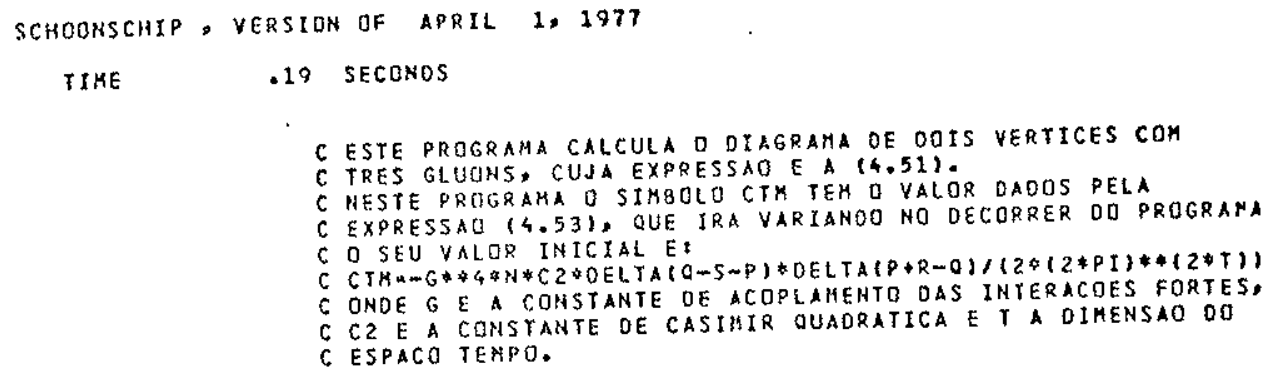

TIME

ceste programa calcula o diagrama oe oots vertices com

C TRES GLUONS, CUJA EXPRESSAOE A $(4,51)$.

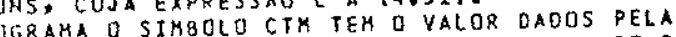

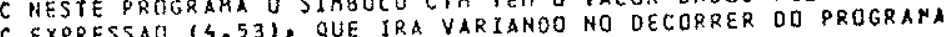

C OSEV YALOR INICIAL E:

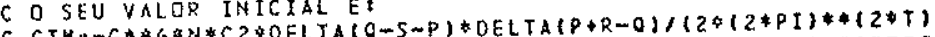

C CTRM-G

C ONDE G C CONSANTE CASIMIR OUADRATICA E T A DIHENSAO DO

C CZE A CONSYANT
C ESPACO TEXPO.

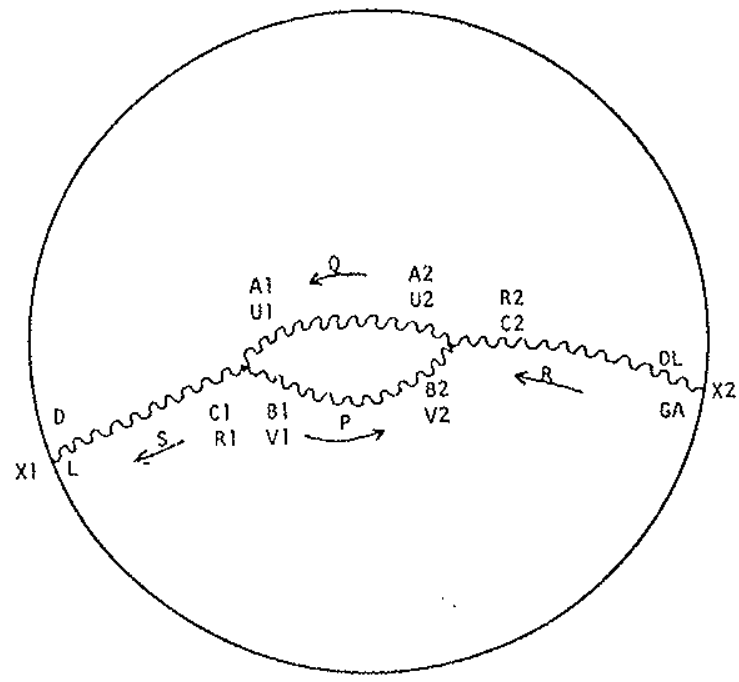

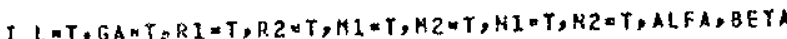

S Y,SS,TSZ

$\checkmark P, 0, R, 5, \times 1, \times 2$

F YER,PRO,GZ,DGZ, EXPS

Z G48\% X)(L) $\times 2(G A) * P R O(R Z, G A, R, R R) * P R O(H 1, H 2,0,00) *$

VER $(0,-S,-P, H 1, R 1, N 1)$

*VER $\{-O R+P, R, M Z, N Z, R Z) \& P R \cap(L, R 2, S, S S) * P R O\{N 1, N 2, P, P P\} * C T M$

*VR

$L 1 \quad I 0, P R Q(R 2+, G A+P+, M I+, R 1+, N 1+)=(Q(R 1)-P(R))) * D(M L, N 1)+$

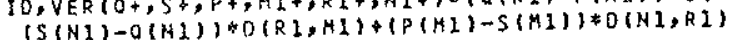

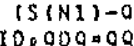

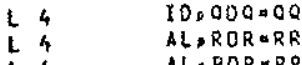

$A L, P D P$ MP

$A L, S D S$ WS

$A L=O O T P R, O(G A+)=S(G A)+P\{G A)$

$A L, O O P P R, O(G A+) \times S(G A)+P(G A)$

ID,OOTPR, R(GAA) SIGA)

$A L, P O P \cap P P$

AR

8 CTA

PEITT NLIST 
PRINT NSYA

PRINT DUTPUT

-YEP

$648=$

$+\mathrm{CTH}$

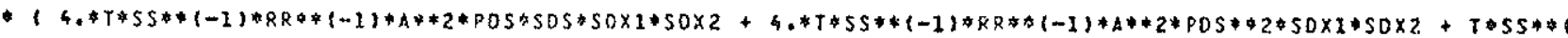
$\# R R *(-1) * A * 2 * S D S * 2 * S D \times 2 * S O \times 2-4 *\} * S S *(-1) * A * P D S * P O \times 2 * S D \times 1-2 * T * S S * *(-2) * A * P D S * S D \times 2 * S O \times 2-2 * * 7 * S S *$ *A*PDX2*SDS*SDX1-T*SS* $\{-1) * A * S D S * S O \times 1 * S O \times 2-4 * * T * R R *(-1\} * A * P D S * P D \times 1 * S C \times 2-2, * T * R R *(-1) * A * P D S * S D \times\} * S$

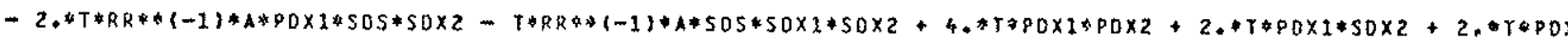

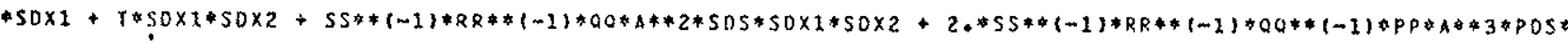
$* S O \times 1 * 50 \times 2-5 S *(-1) * R R *(-1) * 00 * *(-1) * P Q * 2 * A * * 3 * S O S * S O \times 1 * 50 \times 2 * S S *(-1) * R R * *(-1) * Q Q *(-1) * A * 3 * P D S * 2 *$ $* S O \times 2 * S D \times 2+2.5 S *(-1) * R R *(-1) * 0 Q *(* 1) * A * 3 * P O S * * 3 * S O \times 1 * S O \times 2 * S S * *(-1) * R R *(-1) * P P * A * 2 * S D S * S O \times 1 * S O \times 2$

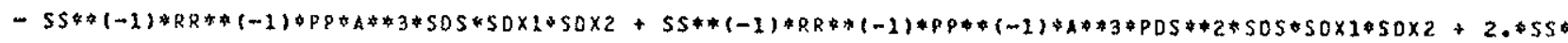

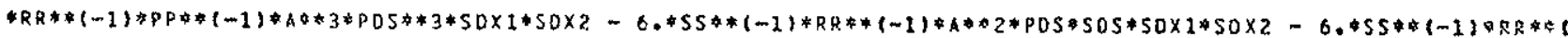
$* A * 2 * 90 S * 2 * S O \times 1 * 5 D \times 2-2 * S S * *(-1) * R R *(-1) * A * 2 * S D S * 2 * S O \times 1 * S O * 2-2 * * 5 S *(-1) * 28 *(-1) * 1 * 3 * P 0 S * 50 S * 50$

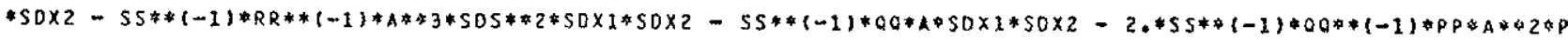

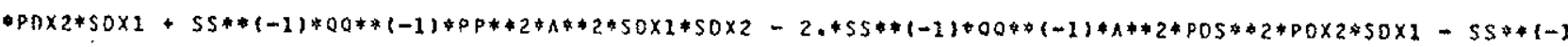

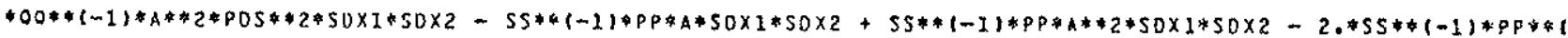

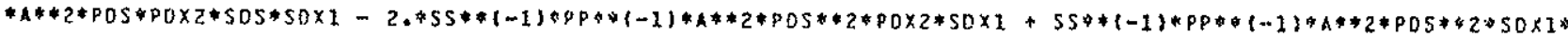

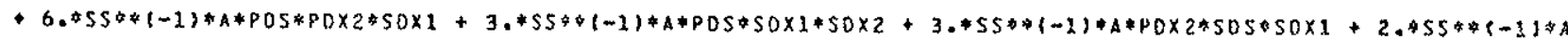

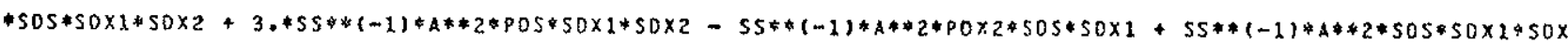

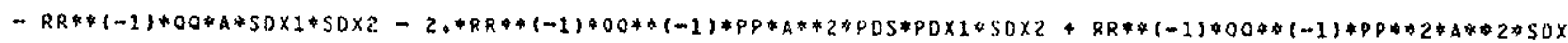

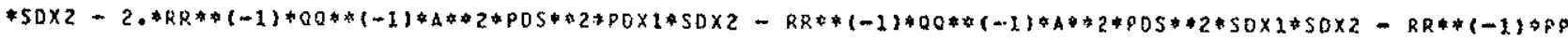

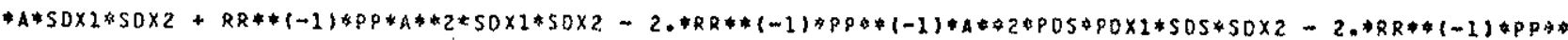

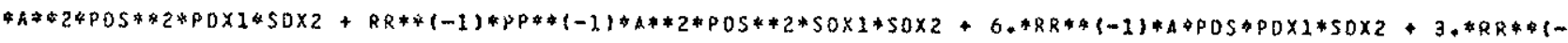

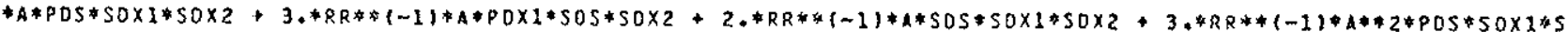

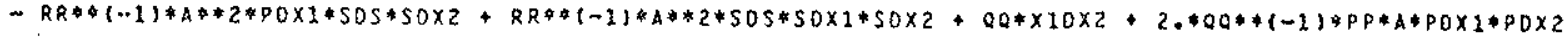

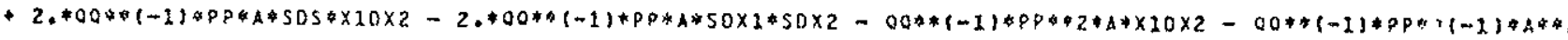

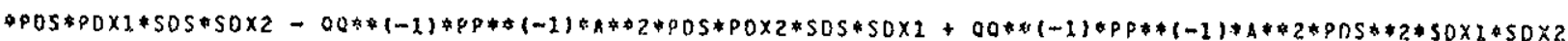

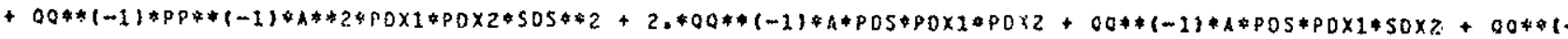

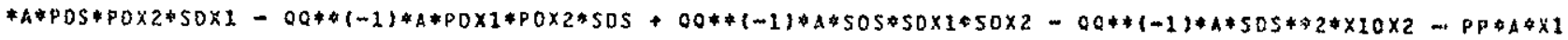

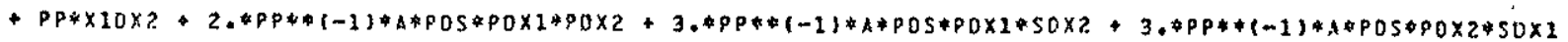

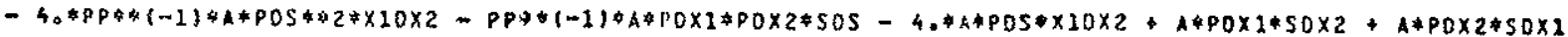




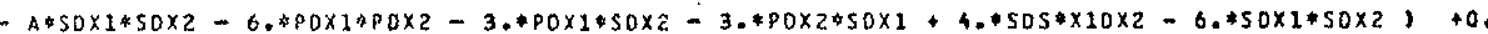

N 8

C INTEGRANOD EH XI(L) E X2(GA) HO CONTORHO, OS TERMOS GUE CONTER S(L) E C SIGA) SE ANULAH.

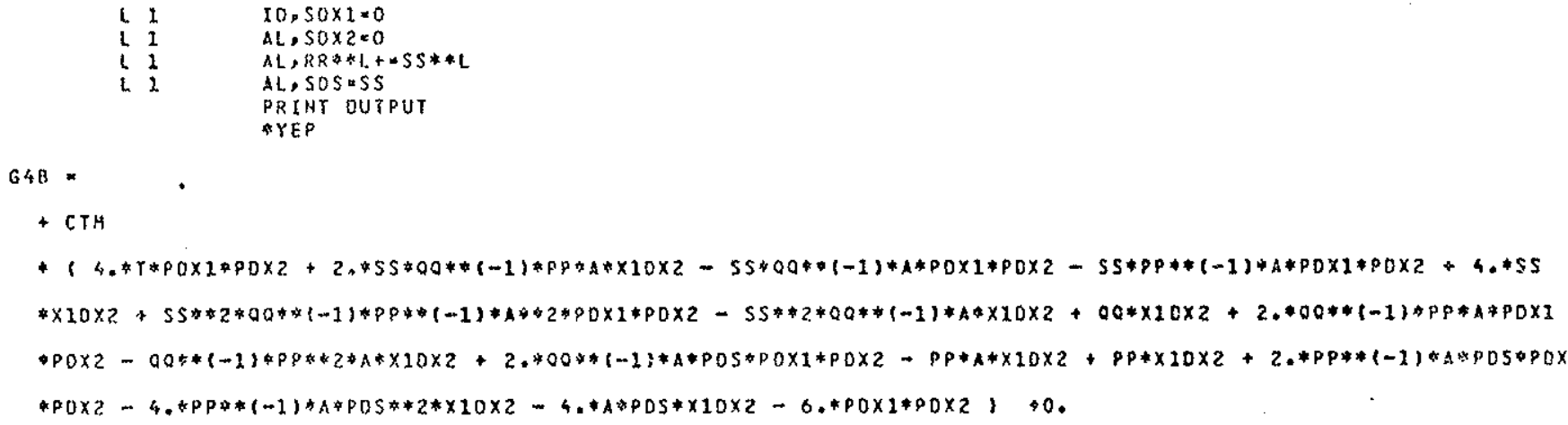


$-\mathrm{C} .4-$

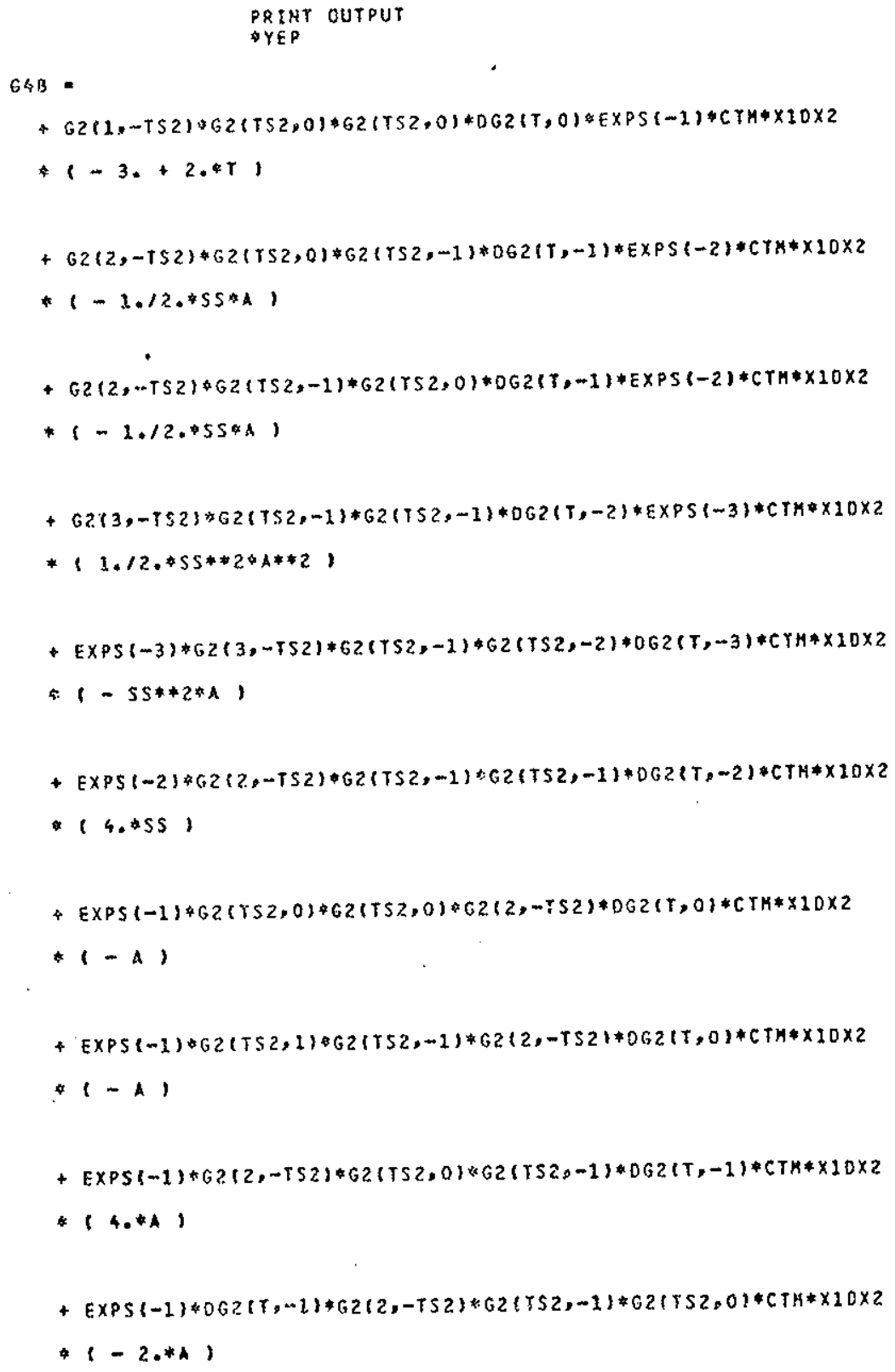




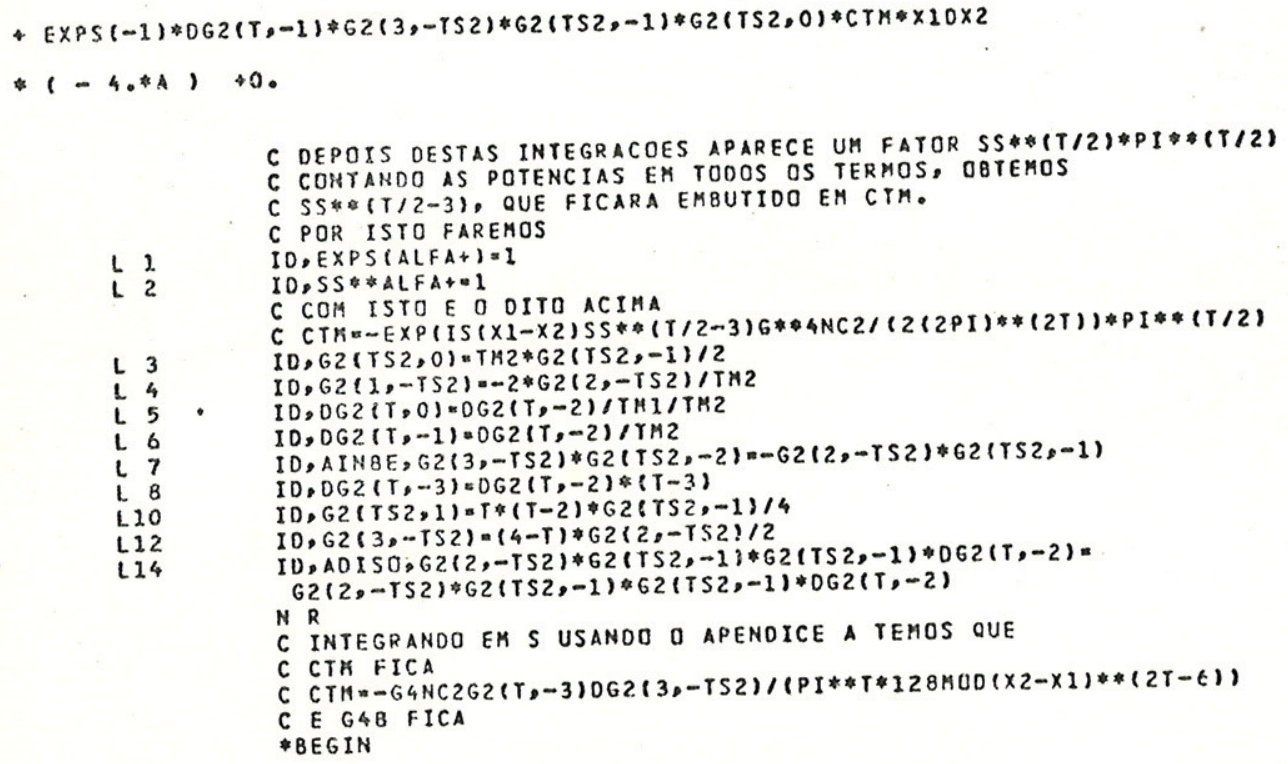

$648=$

$+G 2(2,-T S 2) * G 2(T S 2,-1) * G 2(T S 2,-1) * 0 G 2(T,-2) * C T M * \times 10 \times 2$

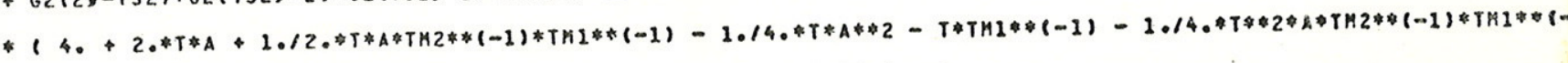

$-13.120 * A-1.140 * A * T H 2 * T H 1 * *(-1)+A * * 2+3.120 * T M 1 * *(-1))+0$. 